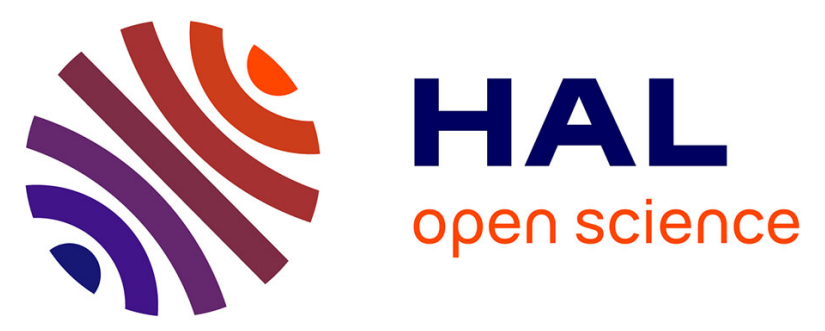

\title{
Analysis and improvement of Brinkman lattice Boltzmann schemes: Bulk, boundary, interface.Similarity and distinctness with finite elements in heterogeneous porous media

\author{
Irina Ginzburg, G. Silva, L. Talon
}

\section{To cite this version:}

Irina Ginzburg, G. Silva, L. Talon. Analysis and improvement of Brinkman lattice Boltzmann schemes: Bulk, boundary, interface.Similarity and distinctness with finite elements in heterogeneous porous media. Physical Review E: Statistical, Nonlinear, and Soft Matter Physics, 2015, 91 (2), 32 p. 10.1103/PhysRevE.91.023307 . hal-01308283

\section{HAL Id: hal-01308283 \\ https://hal.science/hal-01308283}

Submitted on 27 Apr 2016

HAL is a multi-disciplinary open access archive for the deposit and dissemination of scientific research documents, whether they are published or not. The documents may come from teaching and research institutions in France or abroad, or from public or private research centers.
L'archive ouverte pluridisciplinaire HAL, est destinée au dépôt et à la diffusion de documents scientifiques de niveau recherche, publiés ou non, émanant des établissements d'enseignement et de recherche français ou étrangers, des laboratoires publics ou privés. 


\title{
Analysis and improvement of Brinkman lattice Boltzmann schemes: Bulk, boundary, interface. Similarity and distinctness with finite elements in heterogeneous porous media
}

\author{
Irina Ginzburg* \\ Irstea, Antony Regional Centre, HBAN, 1 rue Pierre-Gilles de Gennes CS 10030, 92761 Antony cedex, France \\ Goncalo Silva ${ }^{\dagger}$ \\ Irstea/Cemagref, Antony Regional Centre, HBAN, 1 rue Pierre-Gilles de Gennes CS 10030, 92761 Antony cedex, France \\ Laurent Talon ${ }^{\ddagger}$ \\ CNRS (UMR 7608), Laboratoire FAST, Batiment 502, Campus Universitaire, 91405 Orsay, France
}

(Received 10 September 2014; published 13 February 2015)

\begin{abstract}
This work focuses on the numerical solution of the Stokes-Brinkman equation for a voxel-type porous-media grid, resolved by one to eight spacings per permeability contrast of 1 to 10 orders in magnitude. It is first analytically demonstrated that the lattice Boltzmann method (LBM) and the linear-finite-element method (FEM) both suffer from the viscosity correction induced by the linear variation of the resistance with the velocity. This numerical artefact may lead to an apparent negative viscosity in low-permeable blocks, inducing spurious velocity oscillations. The two-relaxation-times (TRT) LBM may control this effect thanks to free-tunable tworates combination $\Lambda$. Moreover, the Brinkman-force-based BF-TRT schemes may maintain the nondimensional Darcy group and produce viscosity-independent permeability provided that the spatial distribution of $\Lambda$ is fixed independently of the kinematic viscosity. Such a property is lost not only in the BF-BGK scheme but also by "partial bounce-back" TRT gray models, as shown in this work. Further, we propose a consistent and improved IBF-TRT model which vanishes viscosity correction via simple specific adjusting of the viscous-mode relaxation rate to local permeability value. This prevents the model from velocity fluctuations and, in parallel, improves for effective permeability measurements, from porous channel to multidimensions. The framework of our exact analysis employs a symbolic approach developed for both LBM and FEM in single and stratified, unconfined, and bounded channels. It shows that even with similar bulk discretization, BF, IBF, and FEM may manifest quite different velocity profiles on the coarse grids due to their intrinsic contrasts in the setting of interface continuity and no-slip conditions. While FEM enforces them on the grid vertexes, the LBM prescribes them implicitly. We derive effective LBM continuity conditions and show that the heterogeneous viscosity correction impacts them, a property also shared by FEM for shear stress. But, in contrast with FEM, effective velocity conditions in LBM give rise to slip velocity jumps which depend on (i) neighbor permeability values, (ii) resolution, and (iii) control parameter $\Lambda$, ranging its reliable values from Poiseuille bounce-back solution in open flow to zero in Darcy's limit. We suggest an "upscaling" algorithm for $\Lambda$, from multilayers to multidimensions in random extremely dispersive samples. Finally, on the positive side for LBM besides its overall versatility, the implicit boundary layers allow for smooth accommodation of the flat discontinuous Darcy profiles, quite deficient in FEM.
\end{abstract}

DOI: 10.1103/PhysRevE.91.023307

PACS number(s): 02.70.Rr, 02.70.Dh, 02.70.Wz

\section{INTRODUCTION}

In carbonate rocks, believed to host over $50 \%$ of the world's hydrocarbons [1], the fractured poral space is found side by side with very low-permeable micritic domains. Although unresolved on x-ray computed tomography (CT) images, the latter are non-negligible because of their contribution to flow patterns and dispersion. There exists a large variety of double-porosity carbonates, and their effective permeability may vary by several orders of magnitudes, from $10^{-1}$ to $10^{4} \mathrm{mD}$, and the averaged porosity ranges from 0.02 to 0.5 [2]. Typically, one voxel of CT scans representing a few microns of porous sample is mapped onto one node of the computational mesh [3-5], further characterized by its permeability, via typical porosity-permeability relationships.

\footnotetext{
*irina.ginzburg@irstea.fr

†'goncalo.silva@irstea.fr

$\ddagger$ talon@fast.u-psud.fr
}

Although the effective permeability of the entire sample is of the interest, it is determined by the microstructure in an unpredicted way. Upscaling techniques of different complexity aim at assembling multiscale data of distinguished nature from the experimental to numerical [6]. In particular, the direct numerical solution in representative volumes allows an estimation of their effective permeability, with the help of Darcy's law.

Any numerical scheme designed for the mesoscopic solution of the cell-average velocity field $\vec{u}$ needs to accommodate the open (Stokes) flow to Darcy profiles in the presence of the impermeable solid matrix. The Brinkman approach [7] couples the three phases via interface and boundary layers by including the shear into Darcy's equation. This work applies a steady-state Stokes-Brinkman-Darcy equation across the entire domain of isotropic heterogeneous permeability field $k(\vec{r})$ for a positive distribution of porosity function $f(\phi)$ :

$$
\vec{\nabla} \cdot \vec{u}=0, \quad \frac{v \rho_{0}}{k} \vec{u}+\vec{\nabla} P=v_{B} \rho_{0} \Delta \vec{u}, \quad v_{B}=\frac{v}{f(\phi)} .
$$


The Stokes and Darcy regimes are recovered in the two opposite limits, $k \rightarrow \infty$ and $k \rightarrow 0$, respectively. It has been recognized that the effective viscosity $\mu_{B}=v_{B} \rho_{0}$ depends on the interfacial microgeometry. However, its actual relation with porosity $\phi$ is an issue still under debate, as experimental [8], theoretical $[9,10]$, and numerical investigations [11,12] show either increase or decrease of $v_{B}$ with respect to the kinematic viscosity $v$. In default of agreement, the most common semiheuristic rules employ either $f(\phi)=1\left(v_{B}=v\right)$ or $f(\phi)=\phi$. In this context, a related question refers to the interface continuity for the stress-tensor components. The interface force resulting from the resistance was first believed negligible against the shearing. This led Brinkman [7] to adopt usual velocity and stress-continuity conditions. However, more recent works based on either experimental results [13], asymptotic analysis [14-17], or also combining the two approaches [18] have advocated different forms for the so-called stress-jump condition. The two forms of shear-stress difference proportional to the interface slip velocity, BeaversJoseph [13] and Ochoa-Tapia-Whitaker [15,18], are well fitted to the numerical modeling [12] of the microscopic flow over open-porous interface for large porosity $\phi \in[0.7-0.95]$; in smaller porosity of our interest, slip velocity has been found negligibly small, restoring the shear-stress continuity condition. The generic stress-jump condition has been formulated in the framework of the finite-volume method [19]; the shear-stress-jump has been introduced into the lattice Boltzmann scheme [20]. Nevertheless, commonly, the stress continuity is adopted with the local effective viscosity.

To solve the Brinkman equation in heterogeneous media, two numerical approaches compete in versatility: the finite element method (FEM) (see Refs. [21-24] and references herein) and the lattice Boltzmann method (LBM). The LBM can be further subdivided into two main groups. The first one [3,20,25-31] incorporates a momentum sink in the form of the local grid-node drag force. We hereafter refer to this group as the Brinkman-force (BF) model. The second group modifies the population dynamics in a more elegant "kinetic" way, partly reverting the postcollision populations in the spirit of the "partial-bounce-back" rule. In turn, this second group performs the momentum exchange in two ways: (i) nonlocal or link-based [32-34] and (ii) local or grid-node based [35-38]. We will refer to these last algorithms as the "gray" schemes.

Commonly, the LBM Brinkman schemes are validated through model examples allowing for analytical solutions, such as Couette and Poiseuille Stokes-Brinkman porous flow, fluid flow in stratified heterogeneous layers, or partially porous conduits. Further, they are compared with the finite-difference or finite-volume methods for BrinkmanForchheimer flow in channels, lid-driven cavity, or plug flow (see Refs. [5,20,27,28,30,31,33-36,38]). Flow around permeable elliptical cylinder [26,34] was validated with the lubrication semianalytical model [39]. Modeling in realistic porous media has been also addressed in the past decade, for example, two-dimensional (2D) bimodal heterogeneous porous media and 2D fractured media on rectangular mesh [31], 3D samples and CT images with multiple matrix components [3], relative permeability measurements towards water saturation in $3 \mathrm{D}$ cement paste [4], and, very recently, sandstone rocks with different contents of calcite phase per voxel [5]. Additionally, multiscale flow modeling has been coupled with the solute transport for dispersion studies in heterogeneous random 2D media [40] and carbonate samples [41].

However, several reservations can be put forward. First, the benchmark simulations were typically run on very large systems of tens to hundreds of cells per permeability contrast, while in rock modeling, only one node per permeability contrast is often available. Second, all the aforementioned LBM Brinkman schemes, except in Ref. [20], operate with the implicit boundary or interface conditions, and their effective slip or continuity conditions have never been examined. However, as confirmed by the analysis in this work, the latter ones determine the effective accuracy of velocity profiles and permeability measurements on coarse grids. Third, the alarm extends over the fact that the single-relaxation-time BGK operator [42] has been employed for all these simulations. Yet it has been demonstrated in Ref. [29] that, similarly to steady-state LBM solutions of Stokes and Navier-Stokes equations [43-46], the BGK becomes inconsistent for StokesBrinkman and Brinkman-Forchheimer force-based models in the sense that their solutions are not set by the governing Darcy numbers and, eventually, Reynolds number. In other words, the BGK inevitably leads to viscosity-dependent apparent permeability, which is rather inconvenient for LBM applications in realistic soil samples.

In the framework of the BF approach, the minimal consistent collision is the two-relaxation-times TRT operator $[47,48]$. In the BF-TRT model $[29,49]$, the spatial variation of drag force induces an apparent, second-order local correction of the modeled viscosity: $v_{B} \rightarrow v_{B}\left[1+\delta\left(k^{-1}, \Lambda\right)\right]$, where $\Lambda$ is freevalued product of two relaxation functions and $\delta$ is anisotropic, in principle [28,29]. The BGK is the TRT subclass where $\Lambda$ is proportional to $v^{2}$, which explains the loss of the second-order consistency in bulk by the BF-BGK. The apparent viscosity correction has been also derived $[35,38]$ for three gray BGK schemes, on the basis of the effective discretization of channel flow, but it has been missed by the asymptotic analysis [34]. Yet it has been recently recognized in Ref. [49] that in these three gray schemes, $\delta$ remains a function of $v$ even if they cope with the TRT operator. Furthermore, it has been shown that the two schemes [35,38] are matched by the BF-TRT via simple but viscosity-dependent transforms of its free eigenvalue, while the "partial bounce back" scheme [37] needs to transform the two relaxation rates. In the present paper, we develop all these results in length.

We also show that within low-resolved high-permeability contrasts, it becomes impossible to avoid negative apparent viscosities, giving rise to spurious numerical oscillations. To correct for this shortcoming, we introduce an approach, called improved IBF-TRT model, which is designed to vanish viscosity corrections in stratified heterogeneous channels. It appears that it also removes flow fluctuations and outperforms the BF-TRT for permeability measurements in two-dimensional bimodal and random media. The rationale behind IBF is that it automatically increases its viscous relaxation function with a local Darcy number decrease but retains the possibility of the overall control with the help of the free antisymmetric-mode rate. Thereby, a new point is that the effective product of two relaxation functions now varies in space, along with the heterogeneity. 
On the other hand, the extension of our LBM analysis to the linear finite-element method (FEM), for instance, employed by the COMSOL code [23], reveals that the FEM also incorporates specific viscosity correction $\delta\left(k^{-1}\right)$ due to involved discretization force stencil. Although the idea is straightforward, we are not aware of this result in previous studies on FEM. The BF-TRT matches this correction for one particular $\Lambda$, but we will show that the two similar bulk discretizations accommodate very differently highly heterogeneous velocity profiles due to differences in the way they damp or amplify the fluctuations by their respective boundary and interface conditions. This presents the intrinsic contrast between the FEM and LBM: The former prescribes velocity and stress continuity in grid vertexes, and the LBM mimics them implicitly when populations travel over the interface.

In this work we derive the exact underlying macroscopic interface continuity conditions in two model situations: porous inclusions in series and parallel layers. Further, we specify them for BF and IBF and gray schemes. Our methodology combines the recurrence equations [43], which provide the exact formulation for the nonequilibrium components, and the exact microscopic interface conditions [47], which link the equilibrium and nonequilibrium components over the interface. This analysis also covers the bounce-back boundary rule. Thereby, no approximation is involved to construct exact symbolic solutions: microscopic for populations in LBM and macroscopic for velocity in LBM and FEM. Such a solution even applies for one-spacing per material property. In LBM, the perpendicular flow across periodic porous layers will dictate the imperative relation between the microscopic and macroscopic momentum for matching a constant Darcy velocity. The parallel flow in turn will reveal that the stress and slipvelocity undergo $\Lambda$-dependent jumps where velocity raises in the open conduits, resulting in $\Lambda$-dependent permeability overestimates. In Darcy's limit, the LBM accommodates flat branches smoothly, thanks to implicit boundary layers, and thereby avoids strong interface oscillations revealed in FEM, but the dependency $k(\Lambda)$ becomes highly nonlinear. Although the updated IBF model improves the overall accuracy, a pressing question concerns how to select $\Lambda$ reliably, efficiently, and robustly. Our work aims to answer this question as well.

The paper is structured as follows. The TRT operator and BF model are analyzed in Secs. II and III, respectively. The improved IBF model is constructed in Sec. IV and related to a more general "modified" TRT update which also covers the gray schemes. The effective discretization of the FEM in the heterogeneous channel is constructed in Sec. V. The symbolic solutions in channels are extended for all schemes and compared. In Sec. VI, we predict accuracy for permeability measurements in two layers and then extend this analysis to multilayered and highly heterogeneous two-dimensional random distributions, with three principal schemes: BF, IBF, and FEM. Based on this study, a heuristic "upscale" algorithm is proposed to select the optimal $\Lambda$ from multilayers to multidimensions. Finally, Sec. VII concludes the work. Appendix A provides the technical details on the multilayered solutions. Appendix B extends the recurrence equations and their channel solutions to the "modified" TRT update.

\section{THE TRT SCHEME}

This section starts by recalling the generic recurrence equations, interface, and bounce-back closure relations $[43,47]$. The steady-state solutions reproduced by the TRT operator obey them for any equilibrium. We then construct the exact nonequilibrium solution and macroscopic equations in heterogeneous Darcy flow and parallel stratified channel flow. This analysis assumes that the two relaxation rates are constant per material property where the individual (boundary or interface) values can be matched with one grid cell. The interface conditions will account for their exact coupling.

\section{A. The bulk}

The discretized system is defined for the regular grid $\{\vec{r}\}$ sketched in Fig. 1. The velocity vectors $\left\{\vec{c}_{q}\right\}$ interconnect the grid nodes. The velocity set contains $Q$ vectors: one zero, $\vec{c}_{0}=\overrightarrow{0}$, for the immobile population, and $Q_{m}=Q-$ 1 nonzero ones, $\vec{c}_{q}=\left\{c_{q \alpha}, \alpha=1, \ldots, d\right\}$, for the moving populations. Cubic velocity sets [42] with the two moving classes are assumed, e.g., $d 2 Q 9, d 3 Q 15$, and $d 3 Q 19$. The first $Q_{m} / 2$ velocity vectors $\vec{c}_{q}$ are set diametrically opposite with the next $Q_{m} / 2$ vectors $\vec{c}_{\bar{q}}$, and a pair of antiparallel velocities $\left(\vec{c}_{q}, \vec{c}_{\bar{q}}\right)$ is referred to as a link. The combinations $\psi_{q}^{+}=\psi_{\bar{q}}^{+}=\frac{1}{2}\left(\psi_{q}+\psi_{\bar{q}}\right)$ and $\psi_{q}^{-}=-\psi_{\bar{q}}^{-}=\frac{1}{2}\left(\psi_{q}-\psi_{\bar{q}}\right)$ are referred to as the symmetric and antisymmetric components, respectively, for any pair $\left(\psi_{q}, \psi_{\bar{q}}\right): \psi_{q}=\psi_{q}^{+}+\psi_{q}^{-}, \forall q$. The immobile population applies $\psi_{0}^{+}=\psi_{\overline{0}}^{+}=\psi_{0}, \psi_{0}^{-}=\psi_{\overline{0}}^{-}=$ 0 . The unknown variables of the scheme at the node $\vec{r}$ and time $t$ are the components of $Q$ dimensional population vector $\boldsymbol{f}(\vec{r}, t), \boldsymbol{f}=\left\{f_{0},\left(f_{q}, f_{\bar{q}}\right), q=1, \ldots, \frac{Q-1}{2}\right\}$, or, equivalently, its symmetric and antisymmetric components: $\boldsymbol{f}=\left\{f_{0}, f_{q}^{ \pm}, q=1, \ldots, \frac{Q-1}{2}\right\}$. Prescribing two relaxation (a)

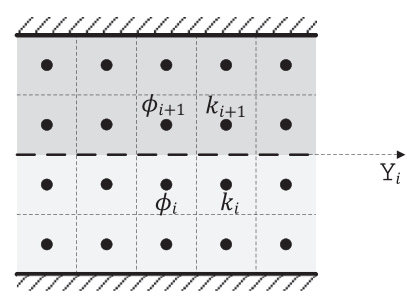

(b)

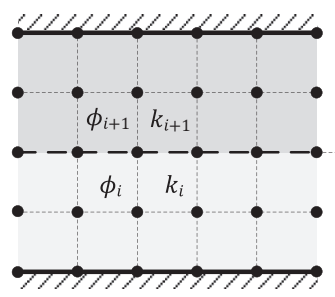

(c)

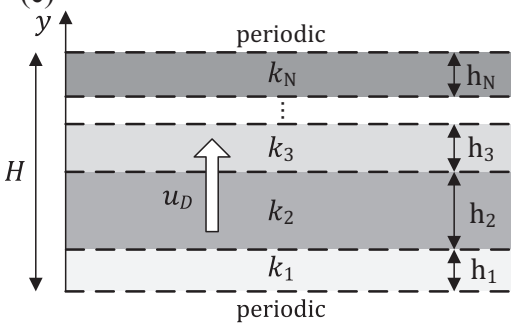

FIG. 1. Sketch for the cell-centered LBM grid (a) and vertex-centered FEM grid (b). Porous layers in series (c). 
parameters $\left.s^{ \pm}(\vec{r}, t) \in\right] 0,2\left[\right.$ and two equilibrium values $e_{q}^{ \pm}(\vec{r}, t)$, the TRT operator [47] updates the population vector $\boldsymbol{f}(\vec{r}, t)$ with two postcollision quantities $g_{q}^{ \pm}(\vec{r}, t)$ per link:

$$
\begin{aligned}
f_{q}\left(\vec{r}+\vec{c}_{q}, t+1\right) & =\tilde{f}_{q}(\vec{r}, t), \quad q=0,1, \ldots, Q-1, \\
\tilde{f}_{0}(\vec{r}, t) & =f_{0}(\vec{r}, t)+g_{0}, \quad g_{0}=-s^{+}\left(f_{0}-e_{0}\right), \\
\tilde{f}_{q}(\vec{r}, t) & =f_{q}(\vec{r}, t)+g_{q}^{+}+g_{q}^{-}, \\
g_{q}^{ \pm} & =-s^{ \pm}\left(f_{q}^{ \pm}-e_{q}^{ \pm}\right), q=1, \ldots, \frac{Q_{m}}{2}, \\
\tilde{f}_{\bar{q}}(\vec{r}, t) & =f_{\bar{q}}(\vec{r}, t)+g_{q}^{+}-g_{q}^{-}, \\
\vec{c}_{\bar{q}} & =-\vec{c}_{q}, \quad q=1, \ldots, \frac{Q_{m}}{2} .
\end{aligned}
$$

The TRT operates with two positive eigenvalue functions $\Lambda^{ \pm}$ and their product $\Lambda$ as follows:

$$
\Lambda^{ \pm}=\left(\frac{1}{s^{ \pm}}-\frac{1}{2}\right), \quad \Lambda=\Lambda^{+} \Lambda^{-} .
$$

A special role for boundary, interface, and bulk accuracy, as well as stability, is played by several specific values of $\Lambda$, such as $\Lambda=\left\{\frac{1}{12}, \frac{1}{8}, \frac{1}{6}, \frac{3}{16}, \frac{1}{4}, \frac{3}{8}, \frac{3}{4}\right\}$, see Refs. [29,45-47,50]. In particular, in Brinkman straight and diagonal channels the $\mathrm{BF}$ annihilates the bulk viscosity correction $\delta$ for $\Lambda=\frac{3}{8}$ and $\Lambda=$ $\frac{3}{4}$, respectively [29]. However, the TRT is not confined to any particular choice and $\Lambda(\vec{r})$ will vary over domain in this work.

In TRT, any external local symmetric or antisymmetric external source terms $M_{q}^{ \pm}(\vec{r}, t)$, added to outgoing populations, can be formally included into equilibrium: $e_{q}^{+} \rightarrow e_{q}^{+}+\left(\Lambda^{+}+\right.$ $\left.\frac{1}{2}\right) M_{q}^{+}$and $e_{q}^{-} \rightarrow e_{q}^{-}+\left(\Lambda^{-}+\frac{1}{2}\right) M_{q}^{-}$. For the purpose of the theoretical analysis, it is proven convenient to express both precollision values $f_{q}(\vec{r}, t)$ and postcollision values $\tilde{f}_{q}(\vec{r}, t)$ as:

$f_{q}(\vec{r}, t)=\left[e_{q}^{+}+e_{q}^{-}-\left(\frac{1}{2}+\Lambda^{+}\right) g_{q}^{+}-\left(\frac{1}{2}+\Lambda^{-}\right) g_{q}^{-}\right](\vec{r}, t), \forall q$,

$\tilde{f}_{q}(\vec{r}, t)=\left[e_{q}^{+}+e_{q}^{-}+\left(\frac{1}{2}-\Lambda^{+}\right) g_{q}^{+}+\left(\frac{1}{2}-\Lambda^{-}\right) g_{q}^{-}\right](\vec{r}, t), \forall q$.

The steady-state recurrence equations [43] of the TRT present specific linear combinations of the evolution equations (2) by substituting there Eqs. (4). They relate the linkwise variation of the equilibrium and nonequilibrium components between them. The first pair of these equations expresses $g_{q}^{ \pm}(\vec{r}):$

$$
\begin{aligned}
& g_{q}^{+}(\vec{r})=\bar{\Delta}_{q} e_{q}^{-}-\Lambda^{-} \bar{\Delta}_{q}^{2} e_{q}^{+}+\left(\Lambda-\frac{1}{4}\right) \bar{\Delta}_{q}^{2} g_{q}^{+}, \\
& g_{q}^{-}(\vec{r})=\bar{\Delta}_{q} e_{q}^{+}-\Lambda^{+} \bar{\Delta}_{q}^{2} e_{q}^{-}+\left(\Lambda-\frac{1}{4}\right) \bar{\Delta}_{q}^{2} g_{q}^{-} .
\end{aligned}
$$

The first and second central linkwise finite differences are, respectively: (i) $\bar{\Delta}_{q} \psi(\vec{r})=\frac{1}{2}\left[\psi\left(\vec{r}+\vec{c}_{q}\right)-\psi\left(\vec{r}-\vec{c}_{q}\right)\right]$ and (ii) $\bar{\Delta}_{q}^{2} \psi(\vec{r})=\psi\left(\vec{r}+\vec{c}_{q}\right)-2 \psi(\vec{r})+\psi\left(\vec{r}-\vec{c}_{q}\right), \forall \psi$. The second pair of the recurrence equations expresses $\bar{\Delta}_{q} g_{q}^{ \pm}(\vec{r})$ :

$$
\begin{aligned}
& \bar{\Delta}_{q} g_{q}^{+}=\bar{\Delta}_{q}^{2} e_{q}^{-}-\Lambda^{-} \bar{\Delta}_{q}^{2} g_{q}^{-}, \\
& \bar{\Delta}_{q} g_{q}^{-}=\bar{\Delta}_{q}^{2} e_{q}^{+}-\Lambda^{+} \bar{\Delta}_{q}^{2} g_{q}^{+} .
\end{aligned}
$$

The combination of Eqs. (5) and (6) will provide a generic solution for $g_{q}^{ \pm}(\vec{r})$ in channel flow. The recurrence equations assume that $\Lambda^{-}$and $\Lambda^{+}$are linkwise constant, meaning their uniform distributions for TRT. In all Stokes-Brinkman schemes we apply linear hydrodynamic equilibrium $[47,48]$ featuring the macroscopic pressure $P$ and momentum $\vec{J}$ in the presence of external forcing $\vec{F}$ :

$$
\begin{aligned}
e_{q}^{+}(\vec{r}) & =P_{q}, \quad P_{q}=t_{q} P(\rho), \quad \rho=f_{0}+2 \sum_{q=1}^{Q_{m} / 2} f_{q}^{+}, \\
e_{0}(\vec{r}) & =\rho-2 \sum_{q=1}^{Q_{m} / 2} e_{q}^{+}, \\
e_{q}^{-}(\vec{r}) & =j_{q}+\Lambda^{-} F_{q}, \quad j_{q}=t_{q}\left(\vec{J} \cdot \vec{c}_{q}\right), \\
\vec{J} & =\vec{J}+\frac{1}{2} \vec{F}, \quad \vec{J}=2 \sum_{q=1}^{Q_{m} / 2} f_{q}^{-} \vec{c}_{q}, \quad \vec{F}=2 \sum_{q=1}^{Q_{m} / 2} F_{q} \vec{c}_{q} .
\end{aligned}
$$

For simplicity, we restrict our analysis to same (isotropic) weights [42] for all terms:

$$
\begin{aligned}
& 2 \sum_{q=1}^{Q_{m} / 2} t_{q} c_{q \alpha} c_{q \beta}=\delta_{\alpha \beta}, \quad \forall \alpha, \beta, \\
& 6 \sum_{q=1}^{Q_{m} / 2} t_{q} c_{q \alpha}^{2} c_{q \beta}^{2}=1, \quad \alpha \neq \beta .
\end{aligned}
$$

We also make use that $6 \sum_{q=1}^{Q_{m} / 2} t_{q} c_{q \alpha}^{2} c_{q \beta}^{4}=1, \alpha \neq \beta$, applies for standard velocity sets. Although the linear relationship $P(\rho)=c_{s}^{2} \rho$ is assumed, $\left.\left.c_{s}^{2} \in\right] 0,1\right]$ is kept as free parameter (further restricted to a velocity-set-dependent stability interval $[45,46,50])$. The conserved momentum is $\vec{J}$, while the macroscopic momentum is $\vec{j}$ [see Eq. (7)]. The macroscopic velocity $\vec{u}$ is formally defined for any scaling parameter $\rho_{0}$ as:

$$
\vec{u}=\frac{\vec{J}}{\rho_{0}} .
$$

Exact mass and momentum conservation equations read, respectively,

$$
\sum_{q=0}^{Q-1} g_{q}^{+}(\vec{r}, t)=0, \quad 2 \sum_{q=1}^{Q_{m} / 2} g_{q}^{-} \vec{c}_{q}(\vec{r}, t)=\vec{F}(\vec{r}, t) .
$$

The zeroth- and first-order moments over Eqs. (5a) and (5b) yield for equilibrium (7):

$$
\bar{\nabla} \cdot \vec{\jmath}=2 \Lambda^{-} \sum_{q=1}^{Q_{m} / 2} \bar{\Delta}_{q}^{2} P_{q}-2\left(\Lambda-\frac{1}{4}\right) \sum_{q=1}^{Q_{m} / 2} \bar{\Delta}_{q}^{2} g_{q}^{+},
$$

$$
\begin{aligned}
\bar{\nabla} P-\vec{F}= & \frac{\Lambda^{+}}{3} \bar{\Delta}^{2} \vec{\jmath}+2 \Lambda \sum_{q=1}^{Q_{m} / 2} \bar{\Delta}_{q}^{2} F_{q} \vec{c}_{q} \\
& -2\left(\Lambda-\frac{1}{4}\right) \sum_{q=1}^{Q_{m} / 2} \bar{\Delta}_{q}^{2} g_{q}^{-} \vec{c}_{q},
\end{aligned}
$$


TABLE I. This table summarizes the BF- and IBF-TRT schemes (2) applied with two relaxation rates $s_{\star}^{ \pm}=1 /\left(\Lambda_{\star}^{ \pm}+\frac{1}{2}\right)$ and equilibrium (7). Input local parameters are the (i) resistance factor $B_{f}=v / k$, (ii) Brinkman viscosity $v_{B}$, and (iii) free positive collision parameter $\Lambda$.

\begin{tabular}{|c|c|c|c|c|c|c|}
\hline Model & Collision & Forcing & Solution $\vec{\jmath}=\rho_{0} \vec{u}$ & $\Lambda_{\star}^{+}=\frac{1}{s_{\star}^{+}}-\frac{1}{2}$ & $\Lambda_{\star}^{-}=\frac{1}{s_{\star}^{-}}-\frac{1}{2}$ & $\Lambda_{\star}=\Lambda_{\star}^{+} \Lambda_{\star}^{-}$ \\
\hline $\mathrm{BF}$ & TRT & $\vec{F}^{p}-B_{f} \vec{\jmath}$ & $\frac{\vec{J}+\vec{F}^{p} / 2}{1+B_{f} / 2}$ & $3 v_{B}$ & $\frac{\Lambda}{3 v_{B}}$ & $\Lambda$ \\
\hline IBF & TRT & $\vec{F}^{p}-B_{f} \vec{\jmath}$ & $\frac{\vec{J}+\vec{F}^{p} / 2}{1+B_{f} / 2}$ & $\frac{9(4+B) \nu_{B}}{4(3+2 B \Lambda)}$ & $\frac{\Lambda}{3 v_{B}}$ & $\frac{3(4+B) \Lambda}{4(3+2 B \Lambda)}$ \\
\hline
\end{tabular}

with

$$
\begin{gathered}
\bar{\nabla} \cdot \vec{J}=2 \sum_{q=1}^{Q_{m} / 2} \bar{\Delta}_{q} j_{q}, \\
\bar{\nabla} P=2 \sum_{q=1}^{Q_{m} / 2} \bar{\Delta}_{q} P_{q} \vec{c}_{q}, \\
\bar{\Delta}^{2} \vec{J}=6 \sum_{q=1}^{Q_{m} / 2} \bar{\Delta}_{q}^{2} j_{q} \vec{c}_{q} .
\end{gathered}
$$

Thus, we express the steady-state macroscopic equations reproduced by the TRT hydrodynamic schemes in terms of the central finite-difference operators for equilibrium and nonequilibrium components, without any approximation.

In $\mathrm{BF}$ and $\mathrm{IBF}$ schemes, considered, respectively, in Secs. III and IV, the external forcing $\vec{F}$ is composed from the uniform flow-driving force $\vec{F}^{p}$ and drag force $-v \vec{J} / k(\vec{r})$; the effective viscosity $v_{B}(\vec{r})=v / f(\phi(\vec{r}))$ and free-tunable parameter $\Lambda(\vec{r})$ are locally prescribed. Assume eigenvalues $s_{\star}^{ \pm}$ in Eq. (2). The BF and IBF differ for choice of the symmetricmode relaxation function $\Lambda_{\star}^{+}=\frac{1}{s_{\star}^{+}}-\frac{1}{2}$. Short summary can be found in Table I. The effective product of two relaxation functions $\Lambda_{\star}^{+}$and $\Lambda_{\star}^{-}=\frac{1}{s_{\star}^{-}}-\frac{1}{2}$ is $\Lambda_{\star}=\Lambda_{\star}^{+} \Lambda_{\star}^{-}$, which is equal to prescribed value $\Lambda_{\star}$ in BF but differs from it in IBF, except for $\Lambda=\frac{3}{8}$, where the two schemes coincide.

\section{B. The interface}

The LBM imposes the interface conditions implicitly, when the two populations cross the interface between the two nodes with the different collision components (see Fig. 1). The TRT satisfies two interface steady-state closure relations at any two nodes $\vec{r}$ and $\vec{r}+\vec{c}_{q}$ :

$$
\begin{aligned}
S_{q}(\vec{r}) & =S_{\bar{q}}\left(\vec{r}+\vec{c}_{q}\right), \\
S_{q}(\vec{r}) & =\left[e_{q}^{+}+\frac{1}{2} g_{q}^{-}-\Lambda^{+} g_{q}^{+}\right](\vec{r}), \\
G_{q}(\vec{r}) & =-G_{\bar{q}}\left(\vec{r}+\vec{c}_{q}\right), \\
G_{q}(\vec{r}) & =\left[e_{q}^{-}+\frac{1}{2} g_{q}^{+}-\Lambda^{-} g_{q}^{-}\right](\vec{r}), \quad \forall \quad e_{q}^{ \pm} .
\end{aligned}
$$

These equations present two linear combinations of the evolution equation (2) (see details in Ref. [47]). Notice that (1) changing $q$ to $\bar{q}$ in $e_{q}^{-}$and $g_{q}^{-}$changes the sign and (2) $\Lambda^{ \pm}$take their local values, i.e., $\Lambda^{ \pm}(\vec{r})$ and $\Lambda^{ \pm}\left(\vec{r}+\vec{c}_{q}\right)$, respectively. A delicate point is that the recurrence equations (5) and (6) apply for constant $\Lambda^{ \pm}$. It will appear that their solution $g_{q}^{ \pm}$can be applied to Eqs. (13) if expressing all finite-difference operators on the virtual linkwise contin- uation of the bulk solutions over the interface, even when the layer has only one grid cell. This way we can derive effective stress and velocity continuity conditions in stratified channels.

\section{The boundary}

The bounce-back reflection is applied in grid boundary node $\vec{r}_{b}$ for cutted link $\vec{c}_{q}$ :

$$
f_{\bar{q}}\left(\vec{r}_{b}, t+1\right)=\tilde{f}_{q}\left(\vec{r}_{b}, t\right) \text { if } \vec{r}_{b}+\vec{c}_{q} \in \text { solid. }
$$

Applying Eq. (4a) for $f_{\bar{q}}\left(\vec{r}_{b}\right)$ and Eq. (4b) for $\tilde{f}_{q}\left(\vec{r}_{b}\right)$, the steadystate closure relation of the bounce-back rule (14) reads

$$
G_{q}\left(\vec{r}_{b}\right)=0, \text { with } G_{q}=e_{q}^{-}+\frac{1}{2} g_{q}^{+}-\Lambda^{-} g_{q}^{-} .
$$

Given that the function $G_{q}(\vec{r})$ is the same as in the continuity condition (13b), the effective bounce-back closure relation can be obtained from the interface analysis.

\section{Porous blocks in "series"}

The Brinkman model assumes continuous macroscopic momentum $\rho_{0} \vec{u}$ over the domain. Obviously, the conserved momentum $\vec{J}$ and the redefined momentum $\vec{J}=\vec{J}+\frac{1}{2} \vec{F}$, employed in Eq. (7), cannot be at the same time continuous on the interface where $\vec{F}$ undergoes the jump. Let us demonstrate that the definition $\vec{J}=\vec{J}+\frac{1}{2} \vec{F}$ is both necessary and sufficient to maintain a one-dimensional constant velocity profile, say, $u_{D}=j_{y} / \rho_{0}$, for piecewise linear continuous pressure distribution $P^{(i)}(y)$. This way we will verify that the constant Darcy velocity in series of porous inclusions (sketched in Fig. 1) is an exact solution of the scheme. Assume $P^{(i)}(y)$ as obeying the momentum equation for constant velocity, $F_{y}^{(i)}=\bar{\nabla}_{y} P^{(i)}$, and for piecewise constant distribution $e_{q}^{-(i)}$ we have:

$$
e_{q}^{+(i)}=t_{q} P^{(i)}, \quad F_{q}^{(i)}=t_{q} \bar{\nabla}_{y} P^{(i)} c_{q y}, \quad \bar{\Delta}_{q} e_{q}^{-(i)}=0 .
$$

Then $\bar{\Delta}_{q}^{2} e_{q}^{ \pm(i)}=0$ and the recurrence equations (5) give:

$$
g_{q}^{+(i)}=0, \quad g_{q}^{-(i)}=\bar{\Delta}_{q} e_{q}^{+}=t_{q} \bar{\nabla}_{y} P^{(i)} c_{q y}=F_{q}^{(i)} .
$$

The stress-continuity condition (13a) reads with

$$
\begin{aligned}
S_{q}^{(i)} & =\left.\left(e_{q}^{+}+\frac{1}{2} g_{q}^{-}\right)\right|_{Y_{i}-\frac{1}{2}} ^{(i)}=\left.t_{q}\left(P^{(i)}+\frac{1}{2} \bar{\nabla}_{y} P^{(i)} c_{q y}\right)\right|_{Y_{i}-\frac{1}{2}} \\
& =t_{q} P^{(i)}\left(Y_{i}\right), \\
S_{\bar{q}}^{(i+1)} & =\left.\left(e_{q}^{+}-\frac{1}{2} g_{q}^{-}\right)\right|_{Y_{i}+\frac{1}{2}} ^{(i+1)}=\left.t_{q}\left(P^{(i+1)}-\frac{1}{2} \bar{\nabla}_{y} P^{(i+1)} c_{q y}\right)\right|_{Y_{i}+\frac{1}{2}} \\
& =t_{q} P^{(i+1)}\left(Y_{i}\right) .
\end{aligned}
$$


Therefore, the scheme maintains continuous piecewise linear pressure distribution. In turn, the two components in velocity-continuity equation (13b) read:

$$
\begin{aligned}
G_{q}^{(i)} & =\left.\left(e_{q}^{-}-\Lambda^{-} g_{q}^{-}\right)\right|_{y=Y_{i}-\frac{1}{2}} \\
& =t_{q} \rho_{0} u_{y}^{(i)} c_{q y}+\left(\Lambda^{-} F_{q}\right)^{(i)}-\left(\Lambda^{-} g_{q}^{-}\right)^{(i)}, \\
-G_{\bar{q}}^{(i+1)} & =\left.\left(e_{q}^{-}-\Lambda^{-} g_{q}^{-}\right)\right|_{y=Y_{i}+\frac{1}{2}} \\
& =t_{q} \rho_{0} u_{y}^{(i+1)} c_{q y}+\left(\Lambda^{-} F_{q}\right)^{(i+1)}-\left(\Lambda^{-} g_{q}^{-}\right)^{(i+1)} .
\end{aligned}
$$

Making use of Eq. (17) in Eq. (18), the continuous velocity condition, $u_{y}^{(i)}=u_{y}^{(i+1)}$, satisfies the system. In case of a drag force $F_{y}^{(i)}=F_{y}^{(p)}-B_{f}^{(i)} \rho_{0} u_{y}$, the scheme matches the Darcy solution $u_{y} \equiv u_{D}$ for harmonic mean $k_{D}$ :

$$
\begin{aligned}
\bar{\nabla}_{y} P^{(i)} & \equiv F_{y}^{(p)}-B_{f}^{(i)} \rho_{0} u_{D}, \quad B_{f}^{(i)}=\frac{\nu}{k_{i}}, \\
F_{y}^{p}-\overline{\nabla_{y} P} & =\frac{\sum_{i} h_{i}\left(F_{y}^{(p)}-\bar{\nabla}_{y} P^{(i)}\right)}{H}=-\rho_{0} u_{D} \nu \frac{\sum_{i} \frac{h_{i}}{k_{i}}}{H}, \\
u_{D} & =-\langle k\rangle \frac{F_{y}^{(p)}-\overline{\nabla_{y} P}}{\rho_{0} \nu}, \quad\langle k\rangle^{-1}=\frac{\sum_{i} \frac{h_{i}}{k_{i}}}{H}, \\
H & =\sum_{i} h_{i} .
\end{aligned}
$$

Remark 1. If the drag force would be computed with $-B_{f} \vec{J}$ instead $-B_{f} \vec{J}$, the numerical solution for $J_{y}$ in periodic series of two inclusions would undergo the interface jumps, as $J_{1} / J_{2}=\left(1-\frac{1}{2} B_{f}^{(2)}\right) /\left(1-\frac{1}{2} B_{f}^{(1)}\right)$ predicted by Eq. (18), and the scheme would be much less stable.

Remark 2. The analysis above is independent of the relaxation rates since the system can be regarded as being at quasiequilibrium, and the linear pressure gradient is compensated by the resistance. Thereby, if the external forcing is perpendicular to periodic system of multi-layers and momentum is redefined, the BF and IBF and gray schemes provide an exact solution (19).

\section{E. Continuous and discretized flow in stratified channels}

\section{Continuous solution}

The streamwise periodic cell is composed of $N$ parallel layers of the thickness $h_{i}$ separated by flat interfaces $y=Y_{i}$. The entire width of the system is $H=\sum_{i=1}^{N} h_{i}$. Inside each layer, $k_{i}$ and $f_{i}(\phi)$ are set piecewise constant. The flow is driven by the constant forcing $F_{x}^{p}=-\bar{\nabla}_{x} P$. We solve Brinkman equation (1) for velocity $u_{x}^{(i)}(y)$

$$
\begin{aligned}
B_{f}^{(i)} \rho_{0} u_{x}^{(i)}(y)-F_{x}^{p} & =v_{B}^{(i)} \rho_{0} \partial_{y y}^{2} u_{x}^{(i)}(y), \quad B_{f}^{(i)}=\frac{v}{k_{i}}, \\
v_{B}^{(i)} & =\frac{v}{f_{i}(\phi)}, \quad y \in\left[Y_{i}-h_{i}, Y_{i}\right] .
\end{aligned}
$$

The system is subject to velocity and shear-stress continuity interface conditions:

$$
u_{x}^{(i)}=\left.u_{x}^{(i+1)}\right|_{y=Y_{i}}, \quad v_{B}^{(i)} \rho_{0} \partial_{y} u_{x}^{(i)}=\left.v_{B}^{(i+1)} \rho_{0} \partial_{y} u_{x}^{(i+1)}\right|_{y=Y_{i}} .
$$

It is convenient to decompose the velocity profile $u_{x}^{(i)}(y)$ into two components: a piecewise-constant Darcy velocity $u_{D}^{(i)}$ and a correction $u_{B}^{(i)}(y)$ :

$$
\begin{aligned}
u_{x}^{(i)}(y) & =u_{D}^{(i)}+u_{B}^{(i)}(y), \quad u_{D}^{(i)}=\frac{\mathrm{Da}_{i}}{\mathrm{Da}} u_{D}, \\
u_{B}^{(i)}(y) & =u_{D} U_{B}^{(i)}\left(y^{\prime}\right), \quad u_{D}=-\frac{\bar{\nabla}_{x} P}{v \rho_{0}} \mathrm{Da} H^{2}, \\
U_{B}^{(i)}\left(y^{\prime}\right) & =-\left(a_{i} e^{\sigma_{i} y^{\prime}}+c_{i} e^{-\sigma_{i} y^{\prime}}\right), \quad y^{\prime}=\frac{y}{H}, \quad \sigma_{i}^{2}=\frac{1}{\mathrm{Da}_{B}^{(i)}}, \\
\mathrm{Da}_{B}^{(i)} & =\frac{\mathrm{Da}_{i}}{f_{i}(\phi)}, \quad \mathrm{Da}_{i}=\frac{k_{i}}{H^{2}} .
\end{aligned}
$$

The reference $\sigma$ value is set with the effective Darcy permeability value $k_{D}$ :

$$
\sigma=\mathrm{Da}^{-1 / 2}, \quad \mathrm{Da}=\frac{k_{D}}{H^{2}}, \quad k_{D}=\left\langle k_{i}\right\rangle=\frac{\sum_{i=1}^{N} h_{i} k_{i}}{H} .
$$

The effective Brinkman permeability value $k_{B}$ is computed from Darcy's law applied to the cell-averaged velocity $\left\langle u_{x}(y)\right\rangle$; its relative correction $k_{r}$ to $k_{D}$ reads

$$
k_{r}=\frac{k_{B}}{k_{D}}-1=\left\langle U_{B}^{(i)}\left(y^{\prime}\right)\right\rangle, \quad k_{B} \in\left[0, k_{D}\right] .
$$

The integration constants $a_{i}$ and $c_{i}$ in Eq. (22) are determined from the interface and boundary conditions. The two configurations sketched in Fig. 2 are periodic [Fig. 2(a)] and bounded [Fig. 2(b)] layers, respectively. In periodic layers along the $y$ axis, Eqs. (21) link adjacent layers. The no-slip condition applies at the horizontal walls in bounded layers.
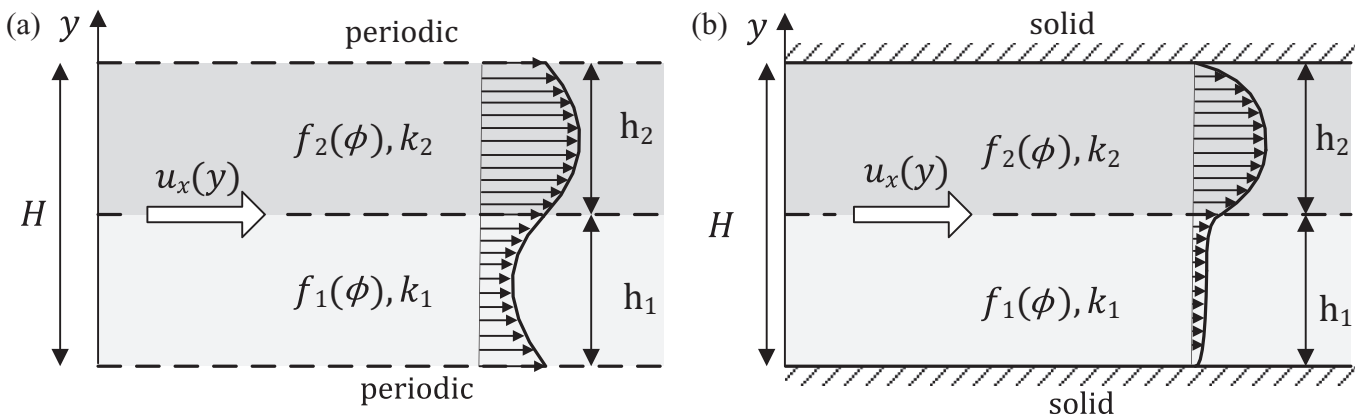

FIG. 2. Periodic (a) and bounded (b) two-layered stratified systems. 

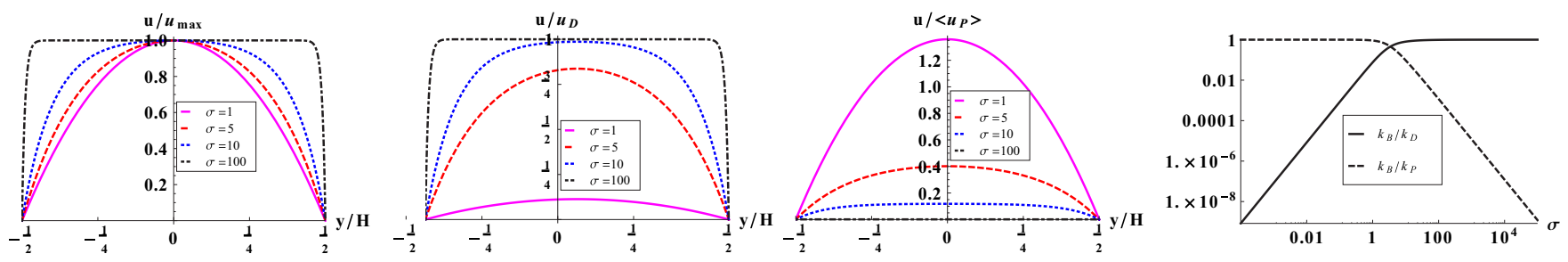

FIG. 3. (Color online) The exact solution (25) in a single channel is plotted when $\sigma=\mathrm{Da}^{-1 / 2}=\left\{1,5,10,10^{2}\right\}$ from the solid (magenta) to dot-dashed (black) lines. The three normalization scalings employed are $u_{\max }, u_{D}$ and averaged Poiseuille value $\left\langle u_{P}\right\rangle$. Last diagram plots effective permeability $k_{B}$ normalized by $k_{D}$ (solid line) and $k_{P}$ (dashed line).

The bounded system reduces to a single porous channel when $k_{i} \equiv k, f_{i}(\phi) \equiv f(\phi), \sigma_{i}^{2}=\sigma$ :

$$
\begin{aligned}
U_{B}\left(y^{\prime}\right) & =-\frac{\cosh \left[\sigma y^{\prime}\right]}{\cosh \left[\frac{\sigma}{2}\right]}, \quad y^{\prime} \in\left[-\frac{1}{2}, \frac{1}{2}\right], \\
k_{r} & =-2 \frac{\tanh \left[\frac{\sigma}{2}\right]}{\sigma} \in[-1,0] .
\end{aligned}
$$

The profiles $U_{B}\left(y^{\prime}\right)$ are plotted in Fig. 3 for several values of $\sigma$ and three different scale factors. The first diagram normalizes all profiles with $u_{\max }$; the second one with $u_{D}$ [cf. Eq. (22)]; the third one adopts $\left\langle u_{P}\right\rangle=-\frac{\bar{\nabla}_{x} P}{v \rho_{0}} \frac{H^{2}}{12}$ : This last scaling is most suitable for the Stokes-Brinkman regime since $u_{D} \rightarrow$ $\infty$ when $\sigma \rightarrow 0$. The three regimes, Stokes-Brinkman with $\sigma \in \approx[0,1]$, intermediate Brinkman, $\sigma \in \approx\left[1,10^{2}-10^{3}\right]$, and Darcy-Brinkman, $\sigma>\approx 10^{2}-10^{3}$, are clearly seen on the basis of permeability (last diagram), where $k_{B}$ varies between $k_{P}=\frac{H^{2}}{12}$ and $k_{D}$. Figure 4 illustrates the exact solution given by Eq. (22) for both two-layered periodic and bounded systems, with the permeability contrast equal to 10 . The last diagram depicts $k_{B}(\sigma) / k_{D}$. In this paper, all solutions are demonstrated for $f_{i}(\phi)=1$ (except for Fig. 13).

\section{Nonequilibrium solution in channel flow}

We apply the recurrence equations to find $g_{q}^{ \pm}$in a straight channel for equilibrium (7) with $\vec{J}=\left\{j_{x}(y), 0\right\}$ and forcing $\vec{F}=\left\{F_{x}(y), 0\right\}$. Two key points are as follows:

(1) In straight-channel flow, $g_{q}^{-}$is set by Eq. (10): $\sum_{q=1}^{Q_{m}} g_{q}^{-} c_{q x}=F_{x}, \sum_{q=1}^{Q_{m}} g_{q}^{-} c_{q y}=0$, along with the streamwise conditions. In the two situations of force-driven or constant-pressure-gradient-driven flow, with $\vec{\nabla} P=\left\{\bar{\nabla}_{x} P, 0\right\}$, the solution reads:

$$
\begin{aligned}
\bar{\nabla}_{x} P & =0, \quad \bar{\Delta}_{q} e_{q}^{+}=0: g_{q}^{-}(y)=3 F_{x}(y) T_{q x}, \\
T_{q x} & =t_{q} c_{q x} c_{q y}^{2}, \\
\bar{\nabla}_{x} P & =\text { const, } \quad \bar{\Delta}_{q} e_{q}^{+}=\bar{\nabla}_{x} P_{q} c_{q x}: \\
g_{q}^{-}(y) & =\bar{\nabla}_{x} P_{q} c_{q x}+3\left(F_{x}(y)-\bar{\nabla}_{x} P\right) T_{q x} .
\end{aligned}
$$

(2) Since $\bar{\Delta}_{q}^{2} e_{q}^{+}=0$ in both situations (26), Eq. (6b) implies:

$$
\bar{\Delta}_{q}^{2} g_{q}^{+}(y)=-\frac{\bar{\Delta}_{q} g_{q}^{-}(y)}{\Lambda^{+}} .
$$

Plugging this relation into Eq. (5a), the generic channel solution for $g_{q}^{+}(y)$ reads:

$$
g_{q}^{+}(y)=\bar{\Delta}_{q} e_{q}^{-}(y)-\frac{\left(\Lambda-\frac{1}{4}\right) \bar{\Delta}_{q} g_{q}^{-}(y)}{\Lambda^{+}} .
$$

After substituting Eqs. (26) into Eq. (28), it takes the form:

$$
g_{q}^{+}(y)=\bar{\Delta}_{q} e_{q}^{-}-\frac{3\left(\Lambda-\frac{1}{4}\right)}{\Lambda^{+}} \bar{\Delta}_{q} F_{x}(y) T_{q x} .
$$

Finally, replacing $e_{q}^{-}$by its definition (7b), $g_{q}^{+}(y)$ becomes

$$
g_{q}^{+}(y)=t_{q} c_{q x}\left(\bar{\Delta}_{q} j_{x}(y)+\left[\Lambda^{-}-\frac{3\left(\Lambda-\frac{1}{4}\right)}{\Lambda^{+}} c_{q y}^{2}\right] \bar{\Delta}_{q} F_{x}(y)\right) .
$$

Altogether, the obtained solution for two components $g_{q}^{ \pm}$is given by Eqs. (26) and (30).
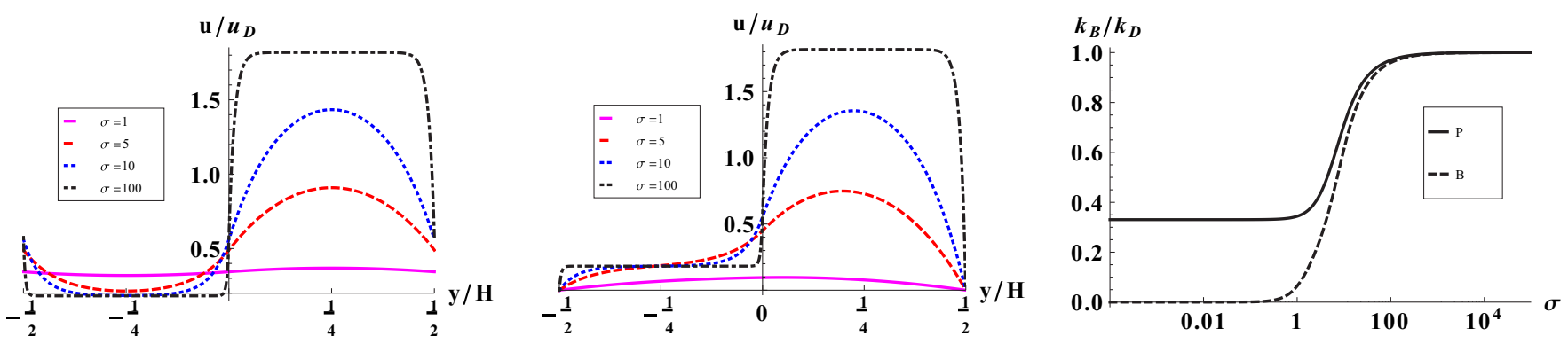

FIG. 4. (Color online) Exact solution (22) in two-layered channels: (left diagram) periodic and (middle diagram) bounded, are plotted for $r_{k}^{2}=k_{1} / k_{2}=10^{-1}$ when $\sigma=\mathrm{Da}^{-1 / 2}=\left\{1,5,10,10^{2}\right\}$ from the solid to the dot-dashed lines. Right diagram: $k_{B} / k_{D}$ in two systems: $\lim _{\sigma \rightarrow 0} \frac{k_{B}}{k_{D}}=\frac{4 r_{k}^{2}}{\left(1+r_{k}^{2}\right)^{2}}$ in the (periodic) system, $\lim _{\sigma \rightarrow 0} \frac{k_{B}}{k_{D}}=0$ in the (bounded) system. 
After substituting Eq. (27) into Eq. (11a) and Eq. (26) into Eq. (11b), the macroscopic equations read for the straight channel as:

$$
\begin{aligned}
\bar{\nabla} \cdot \vec{\jmath} & =2 \frac{\left(\Lambda-\frac{1}{4}\right)}{\Lambda^{+}} \sum_{q=1}^{Q_{m} / 2} \bar{\Delta}_{q} g_{q}^{-} \\
& =6 \frac{\left(\Lambda-\frac{1}{4}\right)}{\Lambda^{+}} \bar{\Delta}_{y} F_{x}(y) \sum_{q=1}^{Q_{m} / 2} t_{q} c_{q x} c_{q y}^{3}=0, \\
\bar{\nabla}_{x} P-F_{x} & =\frac{\Lambda^{+}}{3} \bar{\Delta}_{y}^{2} j_{x}+\frac{\Lambda}{3} \bar{\Delta}_{y}^{2} F_{x}-\left(\Lambda-\frac{1}{4}\right) \bar{\Delta}_{y}^{2} F_{x} \\
& =\frac{\Lambda^{+}}{3} \bar{\Delta}_{y}^{2} j_{x}+\frac{3-8 \Lambda}{12} \bar{\Delta}_{y}^{2} F_{x} .
\end{aligned}
$$

It follows that when $F_{x}$ is neither constant nor linear in space, one should expect the correction to appear in the momentum equation even for straight-channel flow. In Brinkman schemes, this will result in the aforementioned viscosity correction $v_{B} \rightarrow(1+\delta) v_{B}$ since $\bar{\Delta}_{y}^{2} F_{x} \propto \bar{\Delta}_{y}^{2} j_{x}$.

Remark 1 . The momentum equation (31b) has the following equivalent discrete form:

$$
\bar{\nabla}_{x} P-\left(F_{j}+\frac{3-8 \Lambda}{12} \bar{\Delta}_{y}^{2} F_{j}\right)=\frac{\Lambda^{+}}{3} \rho_{0} \bar{\Delta}_{y}^{2} u_{j} .
$$

This corresponds to the three-point force discretization with stencil: $\left\{1+\frac{3-8 \Lambda}{12}, 1-2 \times \frac{3-8 \Lambda}{12}, 1+\frac{3-8 \Lambda}{12}\right\}$. Ahead we will show that the linear-element-based FEM employs the force stencil $\left\{1+\frac{1}{6}, 1-2 \times \frac{1}{6}, 1+\frac{1}{6}\right\}$, which corresponds to $\Lambda=$ $\frac{1}{8}$. This result might appear quite unexpected since the TRT reduces to its discrete equivalents (such as the finite-difference and finite-volumes schemes) for $\Lambda=\frac{1}{4}$, i.e., when the last term vanishes in Eqs. (11) and they become expressed via the variation of the equilibrium (and, hence, macroscopic) components alone [50]. In fact, $\Lambda=\frac{1}{4}$ is shifted to $\Lambda=\frac{1}{8}$ because of the momentum redefinition with the half-forcing.

Remark 2. The steady state is reached when the population flux is constant across the media. In the presence of the external forcing, summation of the population fluxes over the perpendicular section is equivalent to summation of the local values $\vec{j}$ only provided that $\vec{j}$ differs by $\vec{F} / 2$ from $\vec{J}$ [see Ref. [44] and Eq. (7b)]. We have demonstrated that this redefinition is also imperative to match constant velocity in a series of porous blocks.

\section{Discretized solution}

Momentum Eq. (31) for $u_{j}^{(i)}$ will take the same form in all Brinkman schemes:

$$
\begin{gathered}
-F_{x}^{p}+B_{f}^{(i)} \rho_{0} u_{j}^{(i)}=v_{i} \rho_{0} \bar{\Delta}_{y}^{2} u_{j}^{(i)}, \\
v_{i}=v_{B}^{(i)}\left[1+\delta_{i}\left(B_{i}\right)\right], \quad B_{i}=\frac{B_{f}^{(i)}}{v_{B}^{(i)}}=\frac{f_{i}(\phi)}{k_{i}}=\frac{\sigma_{i}^{2}}{H^{2}} .
\end{gathered}
$$

Thereby, $u_{j}^{(i)}$ is decomposed into $u_{D}^{(i)}$ and the correction $u_{D} U_{j}^{(i)}$ [cf. Eq. (22)]:

$$
\begin{aligned}
u_{j}^{(i)}(y) & =u_{D}^{(i)}+u_{D} U_{j}^{(i)}, \\
U_{j}^{(i)} & =-\left(a_{i} r_{i}^{y_{j}}+c_{i} r_{i}^{-y_{j}}\right), \quad y_{j} \in\left[Y_{i}-h_{i}, Y_{i}\right], \\
\text { with } \quad r_{i}\left(B_{i}\right) & =\frac{1+P_{i}}{1-P_{i}}, \quad P_{i}^{2}=\frac{b_{i}^{2}}{1+b_{i}^{2}}, \\
b_{i}^{2} & =\frac{B_{i}}{4\left[1+\delta_{i}\left(B_{i}\right)\right]} .
\end{aligned}
$$

The first difference between Eqs. (20) and (33) is that $\partial_{y y}^{2} u_{x}(y)$ is discretized as $\bar{\Delta}_{y}^{2} u_{j}^{(i)}=u_{j-1}^{(i)}-2 u_{j}^{(i)}+u_{j+1}^{(i)}$, where $\bar{\Delta}_{y}^{2} u_{j}^{(i)}=u_{D} \bar{\Delta}_{y}^{2} U_{j}^{(i)}$ is computed on the same layer solution. The second difference is that the numerical value $\nu_{i}$ differs from the prescribed viscosity value $v_{B}^{(i)}$ by a quantity $\delta_{i}\left(B_{i}\right) \nu_{B}^{(i)}$ as summarized in Table II. Due to linearity of $\delta_{i}$ with $B_{i}, \delta_{i}\left(B_{i}\right)$ reduces as $H^{2}$ with the space resolution and it reduces to zero in the Stokes regime $B_{i}=0$. The key point is that $v_{i}$ may become negative in channel flow with the BF and FEM. This happens when conditions specified in Table II are not satisfied. The negative values $v_{i}$ result in a complex velocity solution in Eq. (34) where the numerical profile manifests spurious oscillations. The BF and FEM differently damp or amplify them according to their interface and boundary conditions. By construction, $\delta_{i}=0$ in IBF and the "ideal" finite-difference IFD scheme. Notice that while of methodological interest, this last scheme is only realized via symbolic solutions. The bounce-back closure relation (15)

\begin{tabular}{|c|c|c|c|c|}
\hline & $\delta_{i}$ & $\alpha_{i}^{+} /\left(1+\delta_{i}\right)$ & $\alpha_{i}^{-} /\left(1+\delta_{i}\right)$ & $v_{i}>0$ \\
\hline $\mathrm{BF}, \Lambda_{i}<\frac{3}{8}$ & $\frac{8 \Lambda_{i}-3}{12} B_{i}$ & 1 & $\frac{16}{3} \Lambda_{i}$ & $B_{i}<\frac{12}{3-8 \Lambda_{i}} \Leftrightarrow \mathrm{Da}_{B}^{(i)}>\frac{3-8 \Lambda_{i}}{12 H^{2}}$ \\
\hline $\mathrm{BF}, \Lambda_{i}>\frac{3}{8}$ & $\frac{8 \Lambda_{i}-3}{12} B_{i}$ & 1 & $\frac{16}{3} \Lambda_{i}$ & $\forall B_{i}, \forall \mathrm{Da}_{B}^{(i)}$ \\
\hline$\left.\mathrm{BF}\right|_{\Lambda_{i}=\frac{3}{8}}, \mathrm{BF}^{-}$ & 0 & 1 & 2 & $\forall B_{i}, \forall \mathrm{Da}_{B}^{(i)}$ \\
\hline $\mathrm{IBF}, \mathrm{BF}^{+}$ & 0 & $\frac{4\left(3+2 \Lambda_{i} B_{i}\right)}{3\left(4+B_{i}\right)}$ & $\frac{16}{3} \Lambda_{i}$ & $\forall B_{i}, \forall \mathrm{Da}_{B}^{(i)}$ \\
\hline IFD & 0 & 1 & 1 & $\forall B_{i}, \forall \mathrm{Da}_{B}^{(i)}$ \\
\hline FEM & $-B_{i} / 6$ & - & - & $B_{i}<6 \Leftrightarrow \mathrm{Da}_{B}^{(i)}>\frac{1}{6 H^{2}}$ \\
\hline
\end{tabular}
reads in a straight channel:

$$
\mathrm{BB}: u_{j}^{(i)} \pm \frac{1}{2} \alpha_{i}^{+} \bar{\Delta}_{y} u_{j}^{(i)}+\left.\frac{1}{8} \alpha_{i}^{-} \bar{\Delta}_{y}^{2} u_{j}^{(i)}\right|_{y_{j}= \pm \frac{H}{2} \mp \frac{1}{2}}=0
$$

with $\bar{\Delta}_{y} u_{j}^{(i)}=\left(u_{j+1}^{(i)}-u_{j-1}^{(i)}\right) / 2$. The coefficients $\alpha_{i}^{ \pm}$are gathered in Table II and, for gray schemes, in Table III. In BF and IBF they reduce to $\left\{\alpha_{i}^{+} \equiv 1, \alpha_{i}^{-} \equiv \frac{16}{3} \Lambda_{i}\right\}$ in Stokes flow $\delta_{i} \equiv 0$, where the Poiseuille profile has zero velocity at $y_{j}= \pm \frac{H}{2}$ only for $\Lambda=\frac{3}{16}$ (see Refs. [44,48]). The new

TABLE II. This table provides effective viscosity coefficient $\delta_{i}\left(B_{i}\right)$ in Eqs. (33) and (36a), coefficients $\alpha_{i}^{ \pm}$of bounce-back closure condition (35), and interface velocity-continuity condition (36b). The last two columns indicate parameter space where $v_{i}$ is positive. 
TABLE III. This table provides coefficients for the $\mathrm{GS}^{\star}, \mathrm{WBS}^{\star}$, and $\mathrm{ZM}^{\star}$ TRT schemes in Eq. (60) and $A^{ \pm}$in Eq. (50), followed by $\delta=\delta^{\star}\left(A^{ \pm}\right)$in Eq. (33) and (36a) and then $\alpha^{ \pm}$in Eqs. (35) and (36b).

\begin{tabular}{|c|c|c|c|c|c|c|c|c|c|}
\hline & $k^{+}$ & $k^{-}$ & $\tilde{k}^{+}$ & $\tilde{k}^{-}$ & $A^{-}$ & $A^{+}$ & $\delta^{\star}\left(A^{ \pm}\right)$ & $\alpha^{+} /\left(1+\delta^{\star}\right)$ & $\alpha^{-} /\left(1+\delta^{\star}\right)$ \\
\hline $\mathrm{GS}^{\star}[35,36]$ & $-n_{s}$ & $n_{s}$ & 0 & 0 & $\frac{B\left(2 \Lambda+\Lambda^{+}\right)}{6+B \Lambda^{+}}$ & 0 & $-\frac{3\left(1+B \Lambda^{+}\right)+2 B \Lambda^{+2}+3 \Lambda(B-8)}{12\left(3+B\left(\Lambda^{+}+\Lambda\right)\right)} B$ & 1 & $-\frac{4\left(B \Lambda^{+2}-12 \Lambda\right)}{3\left(3+B\left(\Lambda^{+}+\Lambda\right)\right)}$ \\
\hline $\mathrm{WBS}^{\star}[37]$ & 0 & $n_{s}$ & $-n_{s}$ & 0 & $\frac{2 B \Lambda}{6+B \Lambda^{+}}$ & $-\frac{B \Lambda^{+}}{6+B \Lambda^{+}}$ & $\frac{\left(\Lambda^{+}-1\right)\left(6+B \Lambda^{+}\right)+6 \Lambda\left(4+B \Lambda^{+}\right)}{6\left(6+B\left(\Lambda^{+}+2 \Lambda\right)\right.} B$ & $\frac{12\left(1+\delta^{\star}\right)}{12+B\left(1+2 \Lambda^{+}\right)}$ & $\frac{32 \Lambda}{6+B\left(\Lambda^{+}+2 \Lambda\right)}$ \\
\hline $\mathrm{ZM}^{\star}[38]$ & 0 & 0 & $-n_{s}$ & $n_{s}$ & $\frac{B\left(2 \Lambda-\Lambda^{+}\right)}{6+B \Lambda^{+}}$ & 0 & $\frac{-9+2 B \Lambda^{+2}-3 \Lambda(B-8)}{12(3+B \Lambda)} B$ & 1 & $\frac{4\left(B \Lambda^{+2}+12 \Lambda\right)}{3(3+B \Lambda)}$ \\
\hline
\end{tabular}

property is that the two coefficients $\alpha_{i}^{ \pm}$scale with the bulk correction $\left(1+\delta_{i}\right)$ in the BF. In combination with $\delta_{i}=0$, the IBF has the same coefficient $\alpha_{i}^{-}=\frac{16 \Lambda_{i}}{3}$ as the TRT Stokes scheme. The IFD yields a second-order Taylor condition with the exact coefficients $\alpha_{i}^{ \pm}=1$. The FEM sets zero velocity in grid vertexes without any approximation.

In stratified channels the two interface relations (13) take the form of the Taylor-type finite-difference approximations of the continuity conditions:

$$
\begin{aligned}
& \left\|v_{i}\left(\bar{\Delta}_{y} u_{j}^{(i)} \pm \frac{1}{2} \bar{\Delta}_{y}^{2} u_{j}^{(i)}\right)\right\|_{Y_{i} \mp \frac{1}{2}}=0, \quad v_{i}=v_{B}^{(i)}\left(1+\delta_{i}\right) \\
& \left\|u_{j}^{(i)} \pm \frac{1}{2} \alpha_{i}^{+} \bar{\Delta}_{y} u_{j}^{(i)}+\frac{1}{8} \alpha_{i}^{-} \bar{\Delta}_{y}^{2} u_{j}^{(i)}\right\|_{Y_{i} \mp \frac{1}{2}}=0
\end{aligned}
$$

Hereafter, $\|\psi\|_{Y_{i} \mp \frac{1}{2}}=0$ implies $\psi_{i}\left(Y_{i}-\frac{1}{2}\right)=\psi_{i+1}\left(Y_{i}+\right.$ $\left.\frac{1}{2}\right)$. Again, all the finite-difference operators apply to the velocity solution of the same layer. The coefficients $\alpha_{i}^{ \pm}$are the same as in Eq. (35). The stress-continuity condition is set with the effective viscosity $v_{i}$.

Symbolic solutions of the TRT schemes then can be constructed by coupling Eqs. (35) and (36) with the bulk solution (34). The closed-form solutions are given by Eq. (A3) for a single channel and by Eqs. (A6) in the two-layered periodic system. The solution applies with $\delta_{i}$ and $\alpha_{i}^{ \pm}$from Tables II and III. The exact agreement between symbolic and numerical results validates our analysis. Once the symbolic solutions have been validated, we may operate with them alone. This enable us to visualize the solution between grid nodes to access the effective location of the boundaries and interfaces and, finally, to predict permeability $k_{B}$ in continuous parameter space.

\section{THE (BRINKMAN-FORCE-BASED) BF SCHEME}

We first derive effective viscosity $v_{i}$ and coefficients in closure relations (35) and (36) in the BF-TRT scheme [29,49]. Here the focus will be put on the spurious oscillations induced by the negative values $v_{i}$ caused by either small permeability or small $\Lambda_{i}$ values. A specific construction is developed in Sec. III C for case $v_{i} \equiv 0$ where solution form (34) does not apply. The BF then provides Darcy's flat profiles accommodated with one or two boundary or interface nodes. Further, we examine the influence of the local, layerwise-constant and/or individual boundary or interface $\Lambda$ values.

\section{A. The bulk}

The BF incorporates the grid-node drag force $\overrightarrow{F^{(r)}}$ directly into Eq. (7):

$$
\begin{aligned}
\mathrm{BF}: F_{q} & =F_{q}^{(r)}+F_{q}^{(p)}, \\
F_{q}^{(r)} & =t_{q}\left(\vec{F}^{(r)} \cdot \vec{c}_{q}\right)=-t_{q} B_{f}\left(\vec{J} \cdot \vec{c}_{q}\right), \quad F_{q}^{(p)}=t_{q}\left(\vec{F}^{p} \cdot \vec{c}_{q}\right), \\
\vec{J}(\vec{r}, t) & =\frac{\vec{J}(\vec{r}, t)+\vec{F}^{p} / 2}{1+B_{f}(\vec{r}) / 2}, \quad B_{f}(\vec{r})=\frac{v}{k(\vec{r})} .
\end{aligned}
$$

Momentum equation (11b) then reads

$$
\begin{aligned}
\bar{\nabla} P-\vec{F}^{p}-\overrightarrow{F^{(r)}}= & v_{B}\left(1+\delta^{i s}\right) \bar{\Delta}^{2} \vec{J} \\
& -2\left(\Lambda-\frac{1}{4}\right) \sum_{q=1}^{Q_{m} / 2} \bar{\Delta}_{q}^{2} g_{q}^{-} \vec{c}_{q}, \\
v_{B}= & \frac{\Lambda^{+}}{3}, \quad \delta^{i s}=-\frac{\Lambda}{3} B, \quad B=\frac{B_{f}}{v_{B}} .
\end{aligned}
$$

The last term $-2\left(\Lambda-\frac{1}{4}\right) \sum_{q=1}^{Q_{m} / 2} \bar{\Delta}_{q}^{2} g_{q}^{-} \vec{c}_{q}$ further modifies the effective viscosity. However, this correction is anisotropic, meaning that its coefficient of $\vec{j}$ depends on the channel orientation with respect to the computational grid. Exact solution for apparent viscosity has been obtained for BGK operator in straight or diagonal channels [28] and, together with approximations for arbitrarily inclined channel, for TRT operator [29]. In the stratified straight channel, Eq. (38) first reduces to Eq. (31b), applying Eq. (26) for $g_{q}^{-}$. After substituting drag force (37), Eq. (31b) reduces to Eq. (33) with $\delta_{i}\left(B_{i}, \Lambda_{i}\right)$ given by Eq. (39):

$$
\delta_{i}\left(B_{i}, \Lambda_{i}\right)=\delta_{i}^{i s}+\left(\Lambda_{i}-\frac{1}{4}\right) B_{i}=\frac{B_{i}\left(8 \Lambda_{i}-3\right)}{12} .
$$

The discretized channel solution has form (34) with

$$
r_{i}\left(B_{i}, \Lambda_{i}\right)=\frac{2 \pm \sqrt{\frac{3 B_{i}}{3+2 B_{i} \Lambda_{i}}}}{2 \mp \sqrt{\frac{3 B_{i}}{3+2 B_{i} \Lambda_{i}}}} .
$$

Thereby, on the one hand, when $\Lambda_{i} \geqslant \frac{3}{8}, \delta_{i} \geqslant 0$, then $v_{i}>0$ and $r_{i}>0$. However, we will observe that this choice is quite inaccurate for poorly resolved interfaces. On the other hand, when $\left.\Lambda_{i} \in\right] 0, \frac{3}{8}\left[\right.$, then $v_{i}>0$ only provided that $B_{i}<\frac{12}{3-8 \Lambda_{i}}$ or, equivalently, $\mathrm{Da}_{B}^{(i)}>\frac{\left(3-8 \Lambda_{i}\right)}{12 H^{2}}$ (see Table II). This means that $\nu_{i}$ is positive independently of $\Lambda_{i}$ when $\left.\Lambda \in\right] 0, \frac{3}{8}$ [ only for relatively large Darcy values: $\mathrm{Da}_{B}^{(i)} \geqslant \frac{1}{4 H^{2}}$.

Remark 1. Since $\mathrm{Da}_{B}^{(i)}=\frac{\mathrm{Da}_{i}}{f_{i}(\phi)}$, smaller Darcy numbers $\mathrm{Da}_{i}$ are available when $f_{i}(\phi)<1$. In particular, if one sets 

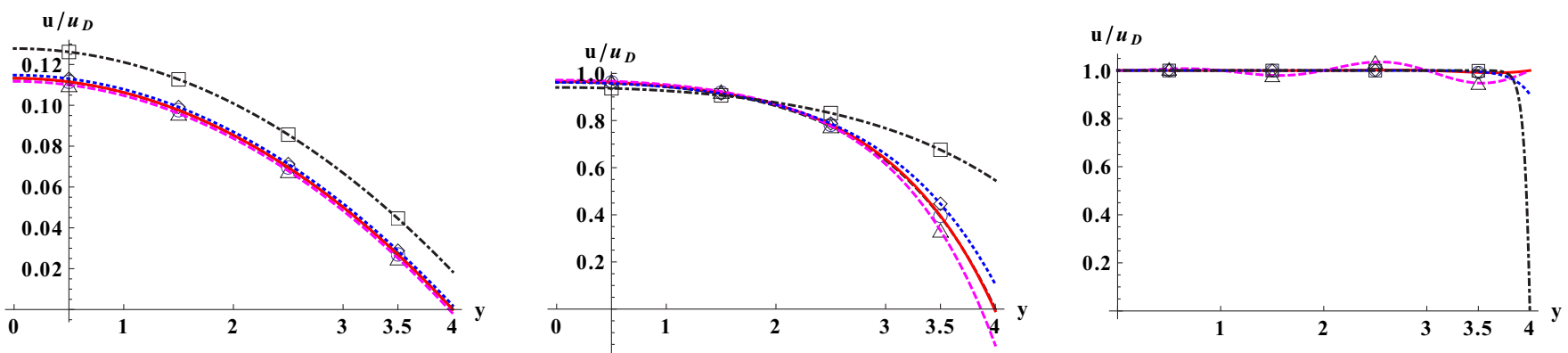

FIG. 5. (Color online) The BF velocity profiles in single channel of width $H=8$ in three regimes (from left to right): $\sigma=1$ (StokesBrinkman regime), $\sigma=8$ (Brinkman), and $\sigma=160$ (Darcy-Brinkman) with $\Lambda=\left\{\frac{1}{512}, \frac{3}{16}, \frac{3}{8}, 2\right\}$ (dashed,solid,dotted,dot-dashed). The triangles, circles, lozenges, and squares mark the grid points. The analytical solution is matched closely by $\Lambda=\frac{3}{16}$ in the two first diagrams; it is dot-dashed (black) in the last diagram.

$f_{i}(\phi)<\frac{4 k_{i}}{H^{2}}$, the constraint vanishes. However, $f_{i}(\phi)$ modifies the effective profiles, and hence $k_{B}$, as can be easily understood from Eq. (25).

Remark 2. Whenever $v_{i}<0$, numerical profiles oscillate. Nevertheless, the computations will show that the BF remains stable.

\section{B. The interface and the boundary}

Here the starting points are Eqs. (13) for the interface and Eq. (15) for the bounce-back. To prescribe $g_{q}^{ \pm}$there, we first substitute Eq. (37) into Eq. (30), which together with Eqs. (26) yields:

$$
\begin{aligned}
& g_{q}^{+(i)}(y) \\
& \quad=t_{q} c_{q x} c_{q y}\left\{1-B_{i}\left[\frac{\Lambda_{i}}{3}-\left(\Lambda_{i}-\frac{1}{4}\right) c_{q y}^{2}\right]\right\} \bar{\Delta}_{y} j_{x}^{(i)}(y), \\
& \Lambda_{i}^{-}\left(F_{q}-g_{q}^{-(i)}\right)(y) \\
& \quad=\Lambda_{i}^{-}\left[F_{x}^{(i)}(y)-\bar{\nabla}_{x} P\right] t_{q} c_{q x}\left(1-3 c_{q y}^{2}\right) .
\end{aligned}
$$

Accounting for $\delta_{i}$ from Eq. (39), Eqs. (41) become for interface or boundary cutted links:

$$
g_{q}^{+(i)}(y)=t_{q} c_{q x} c_{q y}\left(1+\delta_{i}\right) \bar{\Delta}_{y} j_{x}^{(i)}, \quad c_{q y}^{2}=1,
$$

$$
\Lambda_{i}^{-}\left(F_{q}-g_{q}^{-(i)}\right)(y)=\frac{2 \Lambda_{i}}{3} t_{q} c_{q x}\left(1+\delta_{i}\right) \bar{\Delta}_{y}^{2} j_{x}^{(i)}, \quad c_{q y}^{2}=1 .
$$

At the same time, we also substitute $g_{q}^{-}$from Eqs. (26) into stress-continuity condition (13a). We divide it by factor 3 and reorganize as:

$$
\begin{aligned}
\| & -\frac{1}{3} t_{q}\left(P \pm \frac{1}{2} \bar{\nabla}_{x} P c_{q x}\right)+\frac{\Lambda_{i}^{+}}{3} g_{q}^{+} \\
& \pm \frac{1}{2} t_{q}\left[\bar{\nabla}_{x} P-F_{x}^{(i)}(y)\right] c_{q x} c_{q y}^{2} \|_{Y_{i} \mp \frac{1}{2}}=0 .
\end{aligned}
$$

Finally, we account for the pressure continuity condition midway through the interface cutted link: $P+\left.\frac{1}{2} \bar{\nabla}_{x} P c_{q x}\right|_{Y_{i}-\frac{1}{2}}=$ $P-\left.\frac{1}{2} \bar{\nabla}_{x} P c_{q x}\right|_{Y_{i}+\frac{1}{2}}$, and then replace $g_{q}^{+(i)}$ with its solution (42a) and $\left[\bar{\nabla}_{x} P-F_{x}^{(i)}(y)\right]$ with the effective channel bulk equation (33). This yields the interface condition (36a). In turn, Eq. (13b) with Eqs. (42) reduces to Eq. (36b) with (see also in Table II):

$$
\mathrm{BF}: \alpha_{i}^{+}=1+\delta_{i}, \quad \alpha_{i}^{-}=\frac{16}{3} \Lambda_{i}\left(1+\delta_{i}\right) .
$$

The bounce-back closure relation (35) also employs coefficients (44).

Remark The BF differs from the IFD in Table II since the former yields $\delta_{i}=0$ only for $\Lambda_{i}=\frac{3}{8}$, but then $\alpha_{i}^{-}=2$. One exception is the one-node per layer bounded or periodic heterogeneous system (A8) where $\mathrm{BF}\left(\Lambda_{i}=\frac{3}{16}\right)$ reduces to IFD.

Figure 5 shows symbolic BF profiles in three regimes: Stokes-Brinkman $(\sigma=1)$, Brinkman $(\sigma=8)$, and DarcyBrinkman $(\sigma=160)$. They demonstrate that large $\Lambda$ values shift the boundary outside the channel for $\sigma=1$, but the profiles preserve their (parabolic) form. In the Brinkman
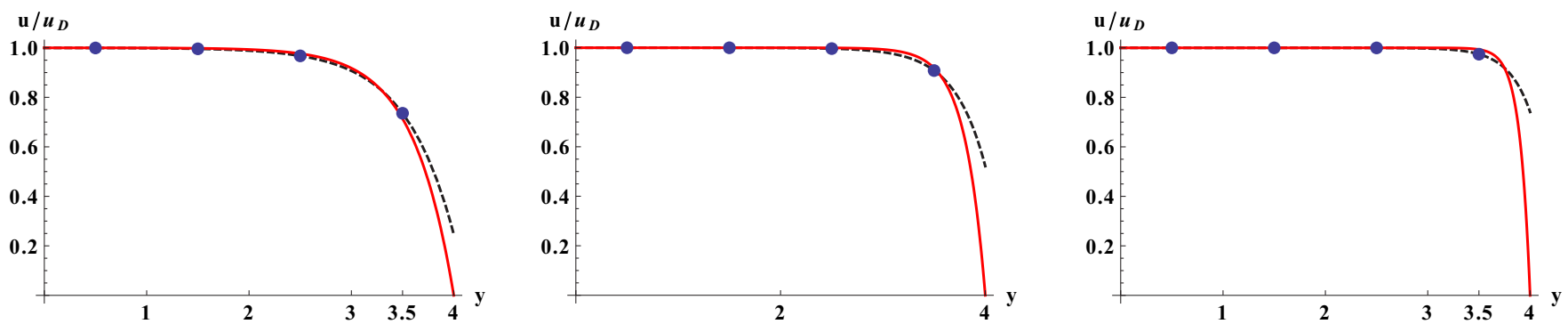

FIG. 6. (Color online) Velocity profiles (dashed) of the "ideal" IFD scheme in a single channel of width $H=8$ in three regimes (from left to right): $\sigma=\{20,40,80\}$. The exact solution is plotted by a solid line. 

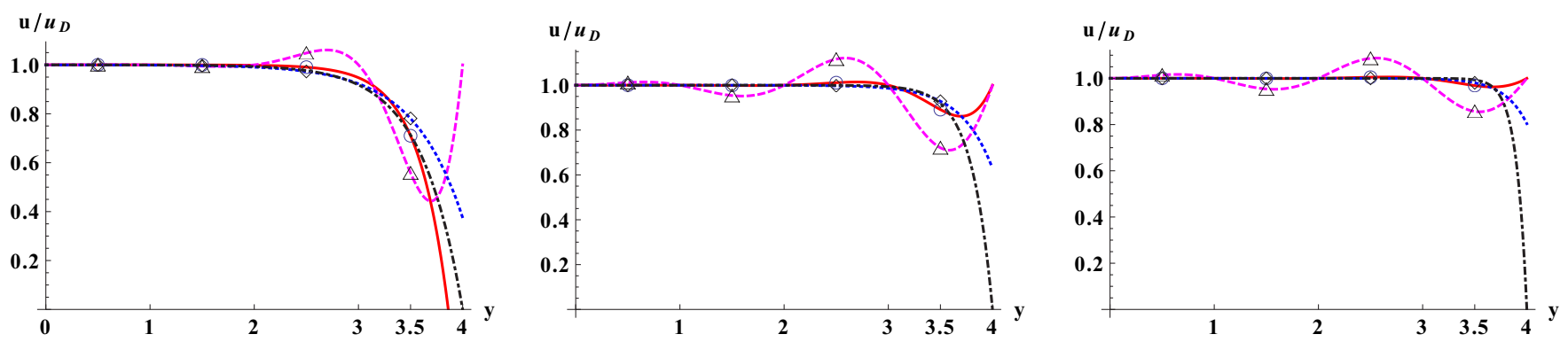

FIG. 7. (Color online) Velocity profiles of the BF in a single porous channel of width $H=8$ are plotted in three regimes (from left to right): $\sigma=\{20,40,80\}$ for $\Lambda=\left\{\frac{1}{512}, \frac{3}{16}, \frac{3}{8}\right\}$ (circle, triangle, lozenge); the exact solution is shown with a dot-dashed line. The profiles are limited to $u>0$.

regime, the profiles deviate by a larger extent from the analytical solution (still well matched for $\Lambda=\frac{3}{16}$ ). In the Darcy regime, $U_{j}$ in last grid nodes $y= \pm \frac{H \pm 1}{2}= \pm \frac{7}{2}$ lies on the exact velocity branch for all $\Lambda$, such that their symbolic continuation towards $y= \pm 4$ is only very slightly decreasing. We also notice the appearance of oscillations for $\Lambda<\frac{3}{8}$, where $v_{i}$ is negative.

Figure 6 plots symbolic velocity profiles when $\sigma=$ $\{20,40,80\}$ with the IFD scheme. Those profiles also do not accommodate the prescribed zero value at $y= \pm \frac{H}{2}$, the discrepancy increases rapidly with $\sigma$, meaning that the secondorder truncated central-difference Taylor approximation (35) is not accurate enough on the sharp Darcy profiles, even for ideal coefficients $\alpha^{ \pm} \equiv 1$. Figure 7 shows that this situation becomes much more complex in BF: $\Lambda=\frac{3}{16}$ noticeably underestimates boundary velocity at $y=4$ for $\sigma=20$, followed by strong oscillations exhibited with $\Lambda=\frac{1}{512}$. Remarkably, their amplitude decreases with $\sigma$ when $|\delta|$ increases. This effect is because of the coupling with the boundary [cf. Eq. (A3)].

Figure 8 illustrates the curious situation in a single channel where we apply a pair of two different values $\left(\Lambda_{1}, \Lambda_{2}\right)$, respectively, for boundary and bulk nodes. It appears that $\left(\frac{3}{16}, \frac{1}{512}\right)$ almost vanishes the oscillations presented by $\left(\frac{1}{512}, \frac{1}{512}\right)$ and the solution becomes only slightly more oscillating in the bulk than for $\left(\frac{3}{16}, \frac{3}{16}\right)$. On the other hand, $\left(\frac{1}{512}, \frac{3}{16}\right)$ only slightly amplifies the boundary oscillation computed with $\left(\frac{3}{16}, \frac{3}{16}\right)$. Finally, the two pairs, $\left\{\Lambda_{1}, \Lambda_{2}\right\}=\left\{\left(\frac{3}{8}, \frac{3}{8}\right),\left(\frac{1}{512}, \frac{3}{8}\right)\right\}$, get nearly the same solutions. Thus, although sufficiently large boundary value $\Lambda_{1}$ suppresses bulk oscillations, a boundary value $\Lambda_{1}$ will not induce them provided that sufficiently large $\Lambda_{2}$ is used.

Figure 9 illustrates the problem of the interface conditions (36) in the periodic channel for the permeability contrast of $10^{2}$ in the Stokes-Brinkman regime $\sigma \approx 2.81$, using two and eight nodes per layer. The two schemes displayed are as follows: the $\operatorname{IFD}$ and $\operatorname{BF}\left(\Lambda=\frac{3}{8}\right)=\operatorname{IBF}\left(\Lambda=\frac{3}{8}\right)$. The IFD demonstrates that the second-order Taylor conditions (36) with exact coefficients, such as $v_{i}=v_{B}^{(i)}$ and $\alpha_{i}^{ \pm}=1$, produce very noticeable interface slip velocity jumps on the coarse grid. The BF with $\Lambda_{i}=\frac{3}{8}$ also yields $\delta_{i}=0$ but it further enlarges the interface velocity jump. This explains why large $\Lambda_{i}$ values should be avoided on coarse grids, even if they vanish the bulk oscillations.

Figure 10 shows that, despite the similarity between the Stokes-Brinkman limit on the open-impermeable interface and Poiseuille solution in the single channel, the interface velocity jump inside open flow is much larger than the bounce-back discrepancy in the location of the no-slip boundary, for the same $\Lambda$ in same resolution. The IBF reduces interface velocity jumps for typical $\Lambda$ values, also improving permeability measurements.

Figure 11 helps us explaining why the permeability observed in Fig. 10 improves for small $\Lambda$. Four possible combinations of the two values $\Lambda_{i} \in\left\{\frac{3}{8}, \frac{1}{512}\right\}$ are applied for permeability contrast $k_{2} / k_{1}=10^{6}$. The most accurate choice is $\Lambda_{1}=\Lambda_{2}=\frac{1}{512}$, followed by $\left(\frac{1}{512}, \frac{3}{8}\right)$ and then $\left(\frac{3}{8}, \frac{1}{512}\right)$ and $\left(\frac{3}{8}, \frac{3}{8}\right)$ (the worst). This figure clearly shows that the improvement in the permeability is due to the decrease of
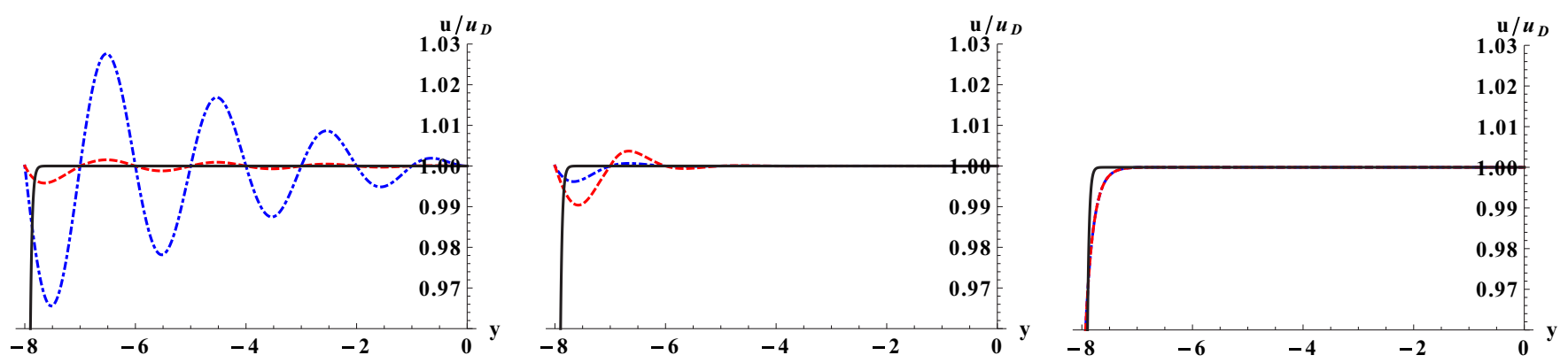

FIG. 8. (Color online) The BF is used with two different values $\left\{\Lambda_{1}, \Lambda_{2}\right\}$ in boundary and bulk nodes, respectively, in a single channel of width $H=16, \sigma \approx 506\left(k=10^{-3}\right)$. The exact solution is plotted with a solid (black) line. Left diagram: $\left(\Lambda_{1}, \Lambda_{2}\right)=\left\{\left(\frac{1}{512}, \frac{1}{512}\right),\left(\frac{3}{16}, \frac{1}{512}\right)\right\}$. Middle diagram: $\left(\Lambda_{1}, \Lambda_{2}\right)=\left\{\left(\frac{3}{16}, \frac{3}{16}\right),\left(\frac{1}{512}, \frac{3}{16}\right)\right\}$. Right diagram: $\left(\Lambda_{1}, \Lambda_{2}\right)=\left\{\left(\frac{3}{8}, \frac{3}{8}\right),\left(\frac{1}{512}, \frac{3}{8}\right)\right\}$. In the three diagrams, the first of two pairs $\left(\Lambda_{1}, \Lambda_{2}\right)$ is dot-dashed (blue) and the second one is dashed (red). 

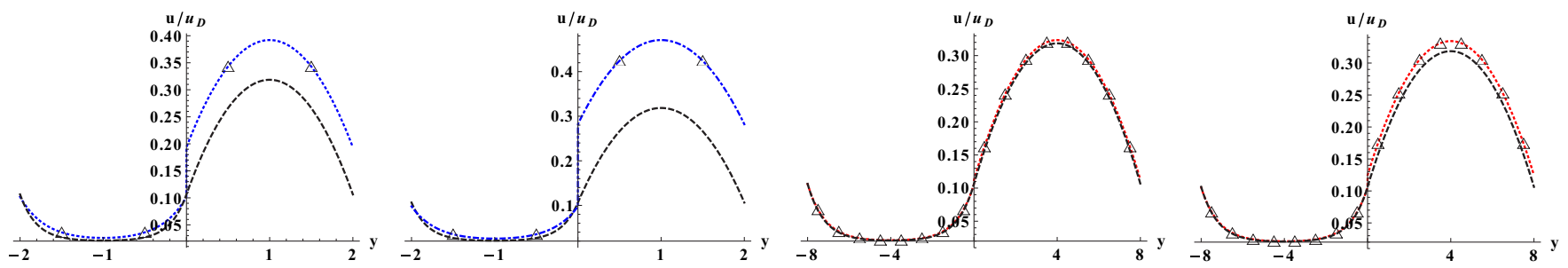

FIG. 9. (Color online) Velocity profiles in a two-layered periodic channel for $k_{2} / k_{1}=10^{2}, \sigma \approx 2.8$ using the "ideal" IFD scheme (first and third diagrams) and $\operatorname{BF}\left(\Lambda=\frac{3}{8}\right)=\operatorname{IBF}\left(\Lambda=\frac{3}{8}\right)$ (second and fourth diagrams). Each layer has two and eight nodes in the first two and last two diagrams, respectively. The analytical solution is shown with a dashed line.

interface velocity jump inside the open layer with the help of small $\Lambda$ value in impermeable layer.

Figure 12 illustrates how the setting individual $\Lambda_{1 i}$ and/or $\Lambda_{2 i}$ values, employed in one or two interface points, may create slip-velocity jumps inside the same layer. This alerts us for the fact that the use of local discontinuous $\Lambda$ distributions have to be adopted with care.

In summary, in the interface between open and porous layers, the BF has worse accuracy than the bounce-back on a solid wall. It follows that using Brinkman schemes through the whole domain with low-permeable "solids" cannot be recommended. The BF has better overall accuracy when a very small value $\Lambda$ is applied, either in all nodes or at least inside the low-permeable layer. In the two cases, solution oscillates for very small Darcy numbers. In addition, the uniform choice of small value $\Lambda$ is quite inaccurate for boundaries in open flow. The strategy IBF presented in Sec. IV may dispel the oscillations for all $\Lambda$ in a straight channel.

\section{Darcy solution for zero effective viscosity}

The effective viscosity $\nu_{i}$ reduces to zero if $\delta_{i}=-1$ :

$$
\begin{aligned}
& B_{i}=\frac{12}{3-8 \Lambda_{i}}, \quad B_{i} \geqslant 4, \quad \text { or } \quad \Lambda_{i}=\frac{3\left(B_{i}-4\right)}{8 B_{i}}, \\
& \left.\left.\Lambda_{i} \in\right] 0, \frac{3}{8}\right], \quad \Lambda_{i} \rightarrow \frac{3}{8} \quad \text { if } \quad B_{i} \rightarrow \infty .
\end{aligned}
$$

We might suggest that the scheme then solves the Darcy equation.

Indeed, in a single channel, the solution consists of the Darcy component $u_{D}$ for all nodes, except the boundary $y_{b}=$ $\pm \frac{H}{2} \mp \frac{1}{2}$ (see Fig. 13), that is:

$$
\begin{aligned}
& u_{j}(y)=u_{D} \quad \text { if } \quad y_{j} \neq y_{b}, \\
& u_{j}\left(y_{b}\right)=\frac{u_{D}}{2}\left(1+\frac{(B-4)}{B}\right)=\frac{B-2}{B} u_{D}, \quad B \geqslant 4 .
\end{aligned}
$$

The bounce-back accommodates the flat profile with the help of the intermediate velocity (46) in the grid-boundary node $y_{b}$. When $B_{i} \rightarrow \infty$, then $u_{j} \rightarrow u_{D}$, meaning that the accommodation value approaches the flat profile. Therefore, as could be expected, the formal use of zero viscosity via $\delta_{i}=-1$ will increase the velocity value in grid boundary node.

Remark 1 . The velocity solution (46) has been derived from the bounce-back closure relation (15) substituting Eqs. (26) for $g_{q}^{-(i)}$ and the following solution for $g_{q}^{+(i)}$ :

$$
g_{q}^{+(i)}=\frac{-F_{x}^{(p)}+B_{f}^{(i)} \rho_{0} u_{i}}{2 v_{B}^{(i)}} t_{q} c_{q x} c_{q y} .
$$

This solution has been derived from Eq. (13a), where $g_{q}^{-(i)}\left(y_{b}\right)$ is given by Eqs. (26) for outgoing populations but the incoming populations from the flat Darcy profile at $y=y_{b}-1$ yield: $g_{q}^{ \pm(i)}\left(y_{b}-1\right)=0$. Then Eq. (47) solves $\frac{1}{2} g_{q}^{-(i)}\left(y_{b}\right)-$ $\Lambda_{i}^{+} g_{q}^{+(i)}\left(y_{b}\right)=0$.

Let us illustrate now the interface situation in the specific limiting case when $v_{i}$ vanishes in the two layers due to the choice (45). The solution of the system is very similar: The velocity profile consists of two Darcy values $u_{D}^{(i)}$ everywhere,
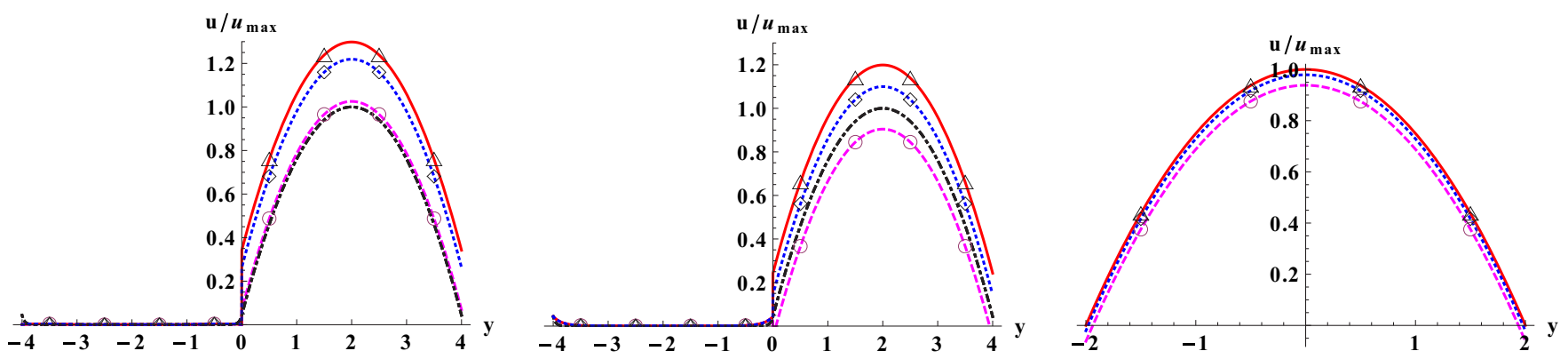

FIG. 10. (Color online) Velocity profiles in Stokes limit $\sigma \approx 0.26, k_{2} / k_{1}=10^{6}$ with the BF (left diagram) and IBF (middle diagram), $\Lambda=\left\{\frac{1}{512}, \frac{1}{8}, \frac{3}{16}\right\}$ [circles (magenta), lozenges (blue), and triangle (red)]. The exact solution is dot-dashed (black). Right diagram: Bounceback Poiseuille solution with the same $\Lambda$ (exact solution is for $\Lambda=\frac{3}{16}$ ). Relative permeability errors: $\operatorname{Err}(k)[\%]=\{3.8,31.3,42.6\}$ in $B F$, $\operatorname{Err}(k)[\%]=\{13.4,14.3,28.5\}$ in $\mathrm{IBF}$, and $\operatorname{Err}(k)[\%]=\{-6.2,0,3.125\}$ in Poiseuille flow. 

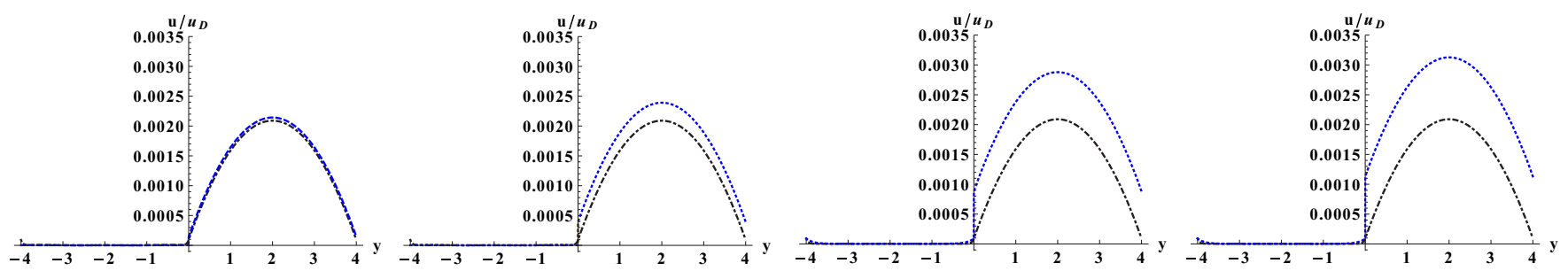

FIG. 11. (Color online) Two periodic layers with $H=8, k_{2} / k_{1}=10^{6}, \sigma=0.26$ when two different values $\Lambda=\left(\Lambda_{1}, \Lambda_{2}\right)$ are used in two layers. The analytical solution is shown in the dash-dotted (black) line. (i) $\left(\frac{1}{512}, \frac{1}{512}\right)$; (ii) $\left(\frac{1}{512}, \frac{3}{8}\right)$; (iii) $\left(\frac{3}{8}, \frac{1}{512}\right)$; (iv) $\left(\frac{3}{8}, \frac{3}{8}\right)$.

except in the two interface accommodation nodes $y=Y \pm \frac{1}{2}$, that is:

$$
\begin{aligned}
u_{j}^{(i)} & =u_{D}^{(i)}=\frac{F^{(p)}}{\rho_{0} B_{f}^{(i)}}, \quad \text { if } \quad y_{j} \neq Y \pm \frac{1}{2}, \\
u^{(1)}\left(Y-\frac{1}{2}\right) & =\left\langle u_{D}\right\rangle+\gamma\left[B_{2} B_{f}^{(1)}+\left(-4+B_{1}\right) B_{f}^{(2)}\right], \\
u^{(2)}\left(Y+\frac{1}{2}\right) & =\left\langle u_{D}\right\rangle-\gamma\left[B_{1} B_{f}^{(2)}+\left(-4+B_{2}\right) B_{f}^{(1)}\right], \\
\text { with } \gamma & =\frac{\rho_{0}\left(B_{f}^{(2)}-B_{f}^{(1)}\right)\left(F^{(p)}-\bar{\nabla}_{x} P\right)}{2 B_{f}^{(1)} B_{f}^{(2)}\left(B_{2} B_{f}^{(1)}+B_{1} B_{f}^{(2)}\right)}, \\
\left\langle u_{D}\right\rangle & =\left(u_{D}^{(1)}+u_{D}^{(2)}\right) / 2 .
\end{aligned}
$$

This solution is illustrated in Fig. 13 for a periodic two-layered channel which can be obtained numerically, e.g., with $\Lambda_{1}=$ $\Lambda_{2}=\Lambda$. This means, from Eq. (45), that the same value $B_{i}=$ $f_{i}(\phi) / k_{i}=B(\Lambda)$ for two different permeability values $k_{i}$ due to $f_{i}(\phi)=k_{i} B(\Lambda)$.

Remark 2. Velocity profile (48) has been constructed similarly to Eq. (47), but now using a four-layer solution where the two interface layers each consist of one node; two interface-to-bulk relations (13a) give Eq. (47). Then we plug these relations, together with Eqs. (26) for $g_{q}^{-(i)}$, into interface condition (13b) and derive velocity solution (48).

To summarize, this constructed solution shows that the BF may run the Darcy equation in different layers thanks to the two-node interface accommodation and, similarly to the boundary, the interface velocities will approach their respective flat profiles with the decrease of the Darcy number.
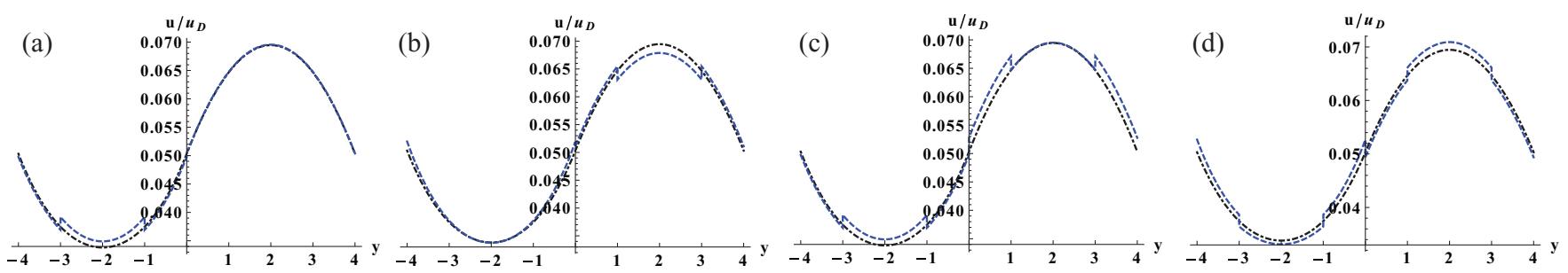

FIG. 12. (Color online) Two periodic layers of permeability contrast $10^{2}$ at $\sigma=0.796$. The analytical solution is shown in dash-dotted (black) and BF profiles are shown in dashed (blue) lines. At the interface nodes, local values $\left\{\Lambda_{1 i}, \Lambda_{2 i}\right\}$ are applied. (a) $\Lambda=\frac{1}{512}$ except $\Lambda_{1 i}=\frac{3}{8}$; (b) $\Lambda=\frac{1}{512}$ except $\Lambda_{2 i}=\frac{3}{8}$; (c) $\Lambda=\frac{1}{512}$ except $\Lambda_{1 i}=\Lambda_{2 i}=\frac{3}{8}$; (d) $\Lambda=\frac{3}{8}$ except $\Lambda_{1 i}=\Lambda_{2 i}=\frac{1}{512}$. 

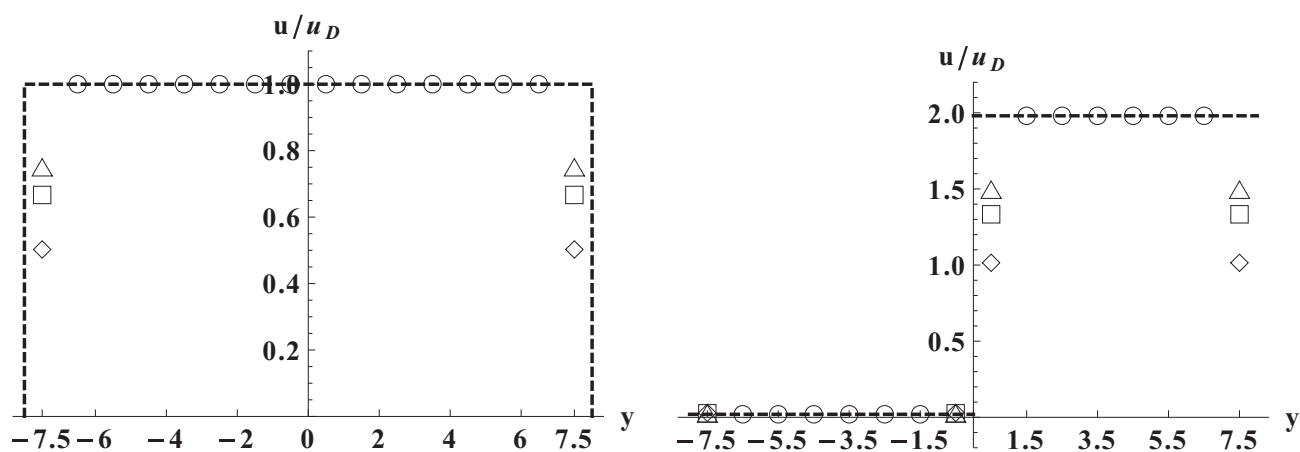

FIG. 13. Exact solutions (46) and (48) in BF when $v_{i}(B, \Lambda)=0$ : The single channel is on the left and the periodic two-layered channel with $k_{2} / k_{1}=10$ is on the right. The solution is $\Lambda$ independent in bulk nodes (circles). In the boundary or interface nodes it is plotted for $\Lambda=\left\{\frac{1}{512}, \frac{1}{8}, \frac{3}{16}\right\}$ (lozenge, square, triangle).

and/or $A^{-} g_{q}^{-}$:

$$
\begin{aligned}
& \tilde{f}_{q}(\vec{r}, t)=f_{q}(\vec{r}, t)+\mathcal{G}_{q}^{+}+\mathcal{G}_{q}^{-}+F_{q}, \quad \mathcal{G}_{q}^{ \pm}=g_{q}^{ \pm}+A^{ \pm} g_{q}^{ \pm}, \\
& g_{q}^{ \pm}=-s^{ \pm} n_{q}^{ \pm}, \quad n_{q}^{ \pm}=f_{q}^{ \pm}-e_{q}^{ \pm}, \quad e_{q}^{-}(\vec{r})=t_{q}\left(\vec{J} \cdot \vec{c}_{q}\right) .
\end{aligned}
$$

It has been first understood [49] that the gray schemes $[35,37,38]$ are covered by Eq. (50) for individual coefficients $A^{ \pm}$. The modified update alters the effective viscosity $\Lambda^{+} / 3$ to $\Lambda^{+}\left(1+\delta_{\star}\left(A^{ \pm}\right)\right) / 3$ and modifies the effective coefficients $\alpha^{ \pm}$in closure relations. These results for gray schemes are shown in Table III. The straightforward idea is to look for two coefficients $A^{ \pm}$which (i) vanish $\delta_{\star}\left(A^{ \pm}\right)$ and (ii) improve the boundary or interface. The relationship $A^{+}\left(A^{-}, B, \Lambda^{+}, \Lambda\right)$ which assures $\delta_{\star}\left(A^{ \pm}\right)=0$ is given by Eq. (B12); the associated coefficients $\alpha^{ \pm}\left(A^{-}, B, \Lambda^{+}, \Lambda\right)$ are given by Eq. (B13). It happens that there is no solution for $A^{+}\left(A^{-}\right)$which would put the two coefficients $\alpha^{ \pm}$equal to 1 , meaning that no modified scheme coincides with the IFD scheme from Table I. In particular, requiring $\alpha^{+}=1$ for $A^{+}=0$ results in a scheme called $\mathrm{BF}^{-}$:

$$
\begin{aligned}
\mathrm{BF}^{-}: A^{+} & =0, \quad A^{-}=\frac{8 \Lambda-3}{3+4 \Lambda^{+}}, \quad \delta_{\star}\left(A^{ \pm}\right)=0, \\
\alpha^{+} & =1, \quad \alpha^{-}=2 .
\end{aligned}
$$

Obviously, for $\Lambda=\frac{3}{8}$ this scheme reduces to $\mathrm{BF}$, together with its coefficients $\alpha^{ \pm}$. The reason for this result is very simple: The BF scheme (2) with $s_{\star}^{ \pm}$coincides with the modified scheme (50) provided that

$$
s_{\star}^{+}=\left(1+A^{+}\right) s^{+}, \quad s_{\star}^{-}=\left(1+A^{-}\right) s^{-} .
$$

As one example, applying Eq. (52) with $A^{ \pm}$from Eq. (51), one gets $s_{\star}^{+}=s^{+}, s_{\star}^{-}=\frac{8 \Lambda^{+}}{3+4 \Lambda^{+}}$, that is $\Lambda_{\star}=\left(\frac{1}{s_{\star}^{+}}-\frac{1}{2}\right)\left(\frac{1}{s_{\star}^{-}}-\frac{1}{2}\right)=$ $\frac{3}{8}$ in $\mathrm{BF}$. This scheme $\operatorname{BF}\left(\Lambda=\frac{3}{8}\right)$ is also obtained applying Eq. (52) to coefficients $A^{ \pm}$of the two gray schemes, GS ${ }^{\star}$ and $\mathrm{ZM}^{\star}$, where $A^{+}=0$, for their specific solution $\Lambda\left(B, \Lambda^{+}\right)$, where $\delta_{\star}=0$ (see Table 4 in Ref. [49]). It also appears that the $\mathrm{BF}^{-}$is equivalent to the aforementioned anisotropic force weights [29] and this property is not restricted to channel flow. The intrinsic reason for this "equivalence" is that all these schemes vanish $\delta_{\star}$ with the help of the antisymmetric component $g_{q}^{-}$alone. Next, it is fruitful to set
$A^{-}=0$ and enforce $\delta_{\star}\left(A^{+}\right)=0$ with $A^{+}$. This scheme is called $\mathrm{BF}^{+}$:

$$
\begin{aligned}
& \mathrm{BF}^{+}: A^{+}=\frac{B \Lambda^{+}(8 \Lambda-3)}{6+12 \Lambda^{+}+3 B \Lambda^{+}+4 B \Lambda}, \\
& A^{-}=0, \quad \delta_{\star}\left(A^{ \pm}\right)=0 .
\end{aligned}
$$

Again, it reduces to BF for $\Lambda=\frac{3}{8}$. Applying Eq. (52) with $A^{+}$ from Eq. (53), we deduce the associated eigenvalue $s_{\star}^{+}$. At the same time, $s_{\star}^{-}$remains unchanged: $s_{\star}^{-}=s^{-}$, since $A^{-}=0$. This eigenvalue choice gives us the IBF scheme from Table I examined in detail below.

In summary, there are two equivalent implementations of the "modified" scheme (50), and IBF or gray schemes in particular. The first one updates populations with Eq. (50), that is, it adds the postcollision corrections to standard TRT update (2) with Brinkman forcing (37). The second one applies the BF in this original form but with locally modified eigenvalues $s_{\star}^{ \pm}$. The two forms are linked via Eqs. (52). Thereby, there also exist two approaches to analyze the modified schemes. The first one applies the extended solution of the recurrence equations developed in Appendix B. Although this might appear unnecessarily complicated, the methodology developed there has the advantage that it can be extended for other problems. The second approach simply updates the eigenvalue functions $\Lambda^{ \pm}$to $\Lambda_{\star}^{ \pm}$in the already derived BF results. This update approach has its subtle points, as is demonstrated by Eq. (59) for IBF model, since now $v_{B} \neq \Lambda_{\star}^{+} / 3$. Finally, there exists an infinite number of combinations $A^{+}\left(A^{-}\right)$, given by Eq. (B12), where $\delta_{\star}\left(A^{ \pm}\right)=0$. Future work needs to explore whether the modified update (50) may motivate another interesting and consistent candidates.

\section{B. The IBF scheme}

The IBF applies the TRT update (2) with equilibrium (7) and forcing (37) but it modifies the eigenvalue of the symmetric component: $s^{+} \rightarrow s_{\star}^{+}=1 /\left(\Lambda_{\star}^{+}+\frac{1}{2}\right.$ ) (see Table I). Namely, assuming that the three quantities mentioned above are locally prescribed: (i) $\Lambda^{+}=3 v_{B}$, (ii) $B_{f}=v / k$, and (iii) $\Lambda$ (meaning $\Lambda^{-}=\Lambda / \Lambda^{+}$), the IBF operates with two local 

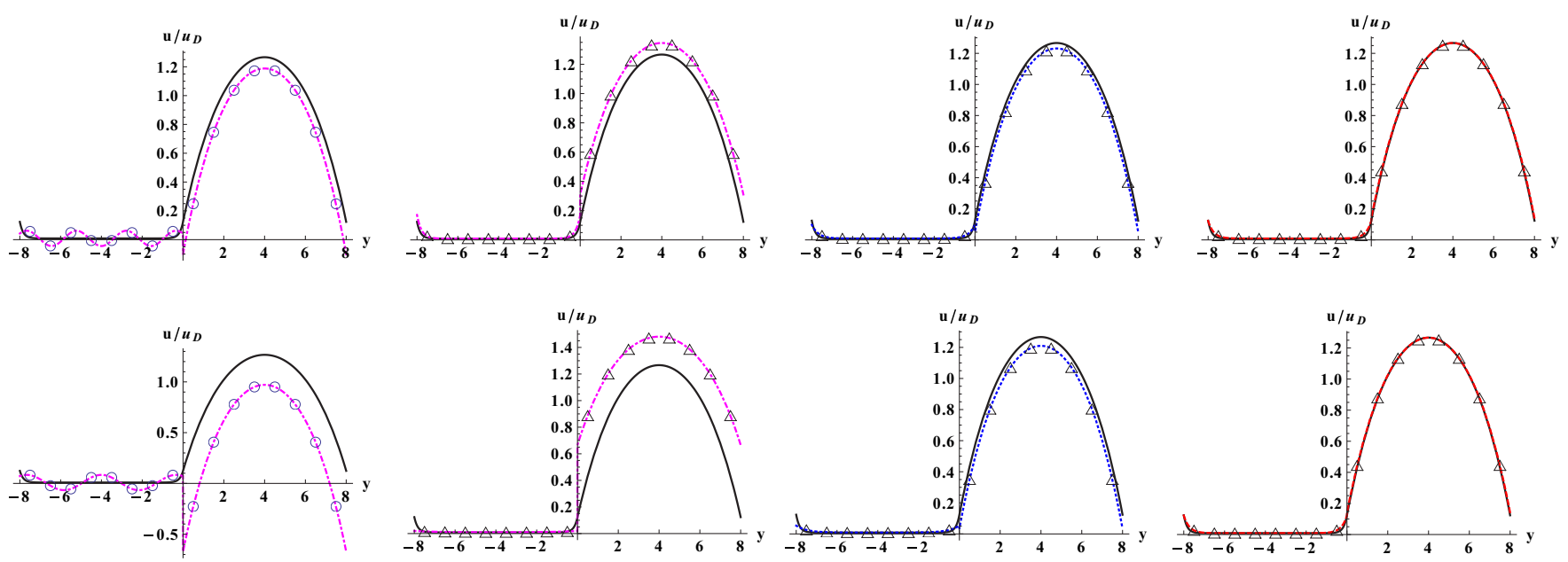

FIG. 14. (Color online) This figure plots two-layered symbolic periodic solutions $u_{x} / u_{D}$ with $k_{1}=0.02(7), k_{2}=6.25$ for four models: $\mathrm{GS}^{\star}, \mathrm{WBS}^{\star}, \mathrm{ZM}^{\star}$, and IBF, with $\Lambda^{+}=3 v_{B}=\frac{1}{2}$ in the top and $\Lambda^{+}=3 v_{B}=\frac{3}{2}$ in the bottom, $\Lambda=\frac{1}{8}$ for all results. The analytical solution is plotted in a solid line (black). Except for the IBF, the nondimensional solutions $u_{x} / u_{D}$ differ for two viscosity values.

functions $\Lambda_{\star}^{ \pm}$:

$$
\begin{aligned}
\mathrm{IBF}: \Lambda_{\star}^{+}\left(B, v_{B}, \Lambda\right) & =\frac{1}{s_{\star}^{+}}-\frac{1}{2}=\frac{9(4+B) v_{B}}{4(3+2 B \Lambda)}, \\
B & =\frac{B_{f}}{v_{B}}=\frac{f(\phi)}{k}, \\
\Lambda_{\star}^{-} & =\frac{1}{s_{\star}^{-}}-\frac{1}{2}=\Lambda^{-}=\frac{\Lambda}{\Lambda^{+}}, \\
\Lambda^{+} & =3 v_{B}=\frac{3 v}{f(\phi)} .
\end{aligned}
$$

The two schemes, BF and IBF, coincide for $\Lambda=\frac{3}{8}$, where $\Lambda_{\star}^{+}=\Lambda^{+}$. We emphasize that $\Lambda_{\star}^{+}$depends linearly on $v_{B}$, like $\Lambda^{+}$in the traditional approach, but the coefficient of proportionality now depends on the local permeability and control parameter $\Lambda$. At the same time, the effective "magic" parameter of IBF is $\Lambda_{\star}(B, \Lambda)$ and it is independent of $v_{B}$ :

$$
\begin{gathered}
\Lambda_{\star}(B, \Lambda)=\Lambda_{\star}^{+} \Lambda_{\star}^{-}=\frac{\Lambda_{\star}^{+}}{\Lambda^{+}} \Lambda=\frac{3(4+B) \Lambda}{4(3+2 B \Lambda)}, \\
\lim _{B \rightarrow 0} \Lambda_{\star}=\Lambda, \quad \lim _{B \rightarrow \infty} \Lambda_{\star}=\frac{3}{8}, \\
\Lambda_{\star}(B, \Lambda)=\Lambda \quad \text { if } \quad \Lambda=\frac{3}{8}, \forall B .
\end{gathered}
$$

That means that even when $\Lambda$ is set constant over the domain, the IBF operates with the heterogeneous distribution $\Lambda_{\star}(\vec{r})$ set by $B(\vec{r})$, except when $\Lambda=\frac{3}{8}$ and $\Lambda_{\star}=\Lambda$. Notice that the IBF remains consistent since $\Lambda_{\star}(\vec{r})$ remains fixed on the given grid together with $B(\vec{r})$ and $\Lambda(\vec{r})$ due to the linearity of function $\Lambda_{\star}^{+}\left(\Lambda^{+}\right)$. This is confirmed by the viscosityindependent nondimensional Brinkman profiles in Fig. 14. The modified $\Lambda_{\star}^{+}$and the traditional $\Lambda^{+}=3 v_{B}$ present the following interesting limiting relations:

$$
\begin{aligned}
& \lim _{B \rightarrow 0} \frac{\Lambda_{\star}^{+}}{\Lambda^{+}}=1, \quad \lim _{B \rightarrow \infty} \frac{\Lambda_{\star}^{+}}{\Lambda^{+}}=\frac{3}{8 \Lambda}, \\
& \lim _{\Lambda \rightarrow 0} \frac{\Lambda_{\star}^{+}}{\Lambda^{+}}=\frac{4+B}{4}, \quad \lim _{B \rightarrow \infty, \Lambda \rightarrow 0} \frac{\Lambda_{\star}^{+}}{\Lambda^{+}}=\infty .
\end{aligned}
$$

This last relation is consistent with the fact that, in the Darcy limit $\sigma \rightarrow \infty$, the IBF compensates negative infinite correction $\delta$ of the standard scheme. In fact, in the straight channel, the IBF has the following coefficients in Eq. (33) and boundary or interface relations [(35) and (36)]:

$$
\begin{aligned}
\delta & \equiv 0, \quad \text { then } \quad r(B)=\frac{1}{2}(2+B \pm \sqrt{B} \sqrt{4+B}), \\
\alpha^{+} & =1+\frac{B(8 \Lambda-3)}{3(4+B)}=\frac{\Lambda}{\Lambda_{\star}}=\frac{\Lambda^{+}}{\Lambda_{\star}^{+}}, \\
\lim _{B \rightarrow 0} \alpha^{+} & =1, \quad \lim _{B \rightarrow \infty} \alpha^{+}=\frac{8}{3} \Lambda, \\
\alpha^{-} & =\frac{16}{3} \Lambda:\left.\alpha^{-}\right|_{\Lambda=\frac{3}{16}}=1 .
\end{aligned}
$$

The IBF has a zero-viscosity correction $\delta=0$ in the straight channel. This can be readily obtained by using Eq. (11b) with Eq. (26) for $\Lambda_{\star}^{ \pm}$:

$$
\begin{aligned}
& \bar{\nabla} P-\vec{F}^{p}-\overrightarrow{F^{(r)}} \\
& \quad=\left[\frac{\Lambda_{\star}^{+}}{3}-B_{f} \frac{\Lambda_{\star}^{-} \Lambda_{\star}^{+}}{3}+B_{f}\left(\Lambda_{\star}-\frac{1}{4}\right)\right] \bar{\Delta}^{2} \vec{J} .
\end{aligned}
$$

The coefficient of $\bar{\Delta}^{2} \vec{j}$ corresponds to the effective numerical viscosity. Equating it to the prescribed viscosity $\Lambda^{+} / 3=v_{B}$, one gets the solution for $\Lambda_{\star}^{+}\left(B, v_{B}, \Lambda\right)$ given by Eq. (54). The key point is that the IBF operates with $B=B_{f} / v_{B}$ where $v_{B} \neq \frac{\Lambda_{*}^{+}}{3}$. Due to this one cannot obtain the coefficient $\alpha^{+}$in Eq. (57) simply by replacing $\delta$ by zero in Eq. (42a). Nevertheless, one obtains it from generic equation (30), which in the presence of the drag force there and restricted to the cutted links $c_{q y}^{2}=1$ reads:

$$
\begin{aligned}
g_{q}^{+}(y) & =\alpha^{+} t_{q} c_{q x} \bar{\Delta}_{q} j_{x}(y), \\
\alpha^{+} & =1-B_{f}\left[\Lambda_{\star}^{-}-\frac{3\left(\Lambda_{\star}-\frac{1}{4}\right)}{\Lambda_{\star}^{+}}\right] .
\end{aligned}
$$

Replacing again $\Lambda_{\star}^{-}$with $\Lambda^{-}$and $\Lambda_{\star}$ with $\Lambda_{\star}^{+} \Lambda^{-}$and substituting Eq. (54) for $\Lambda_{\star}^{+}$, Eq. (59) gives Eq. (57) for $\alpha^{+}$. 

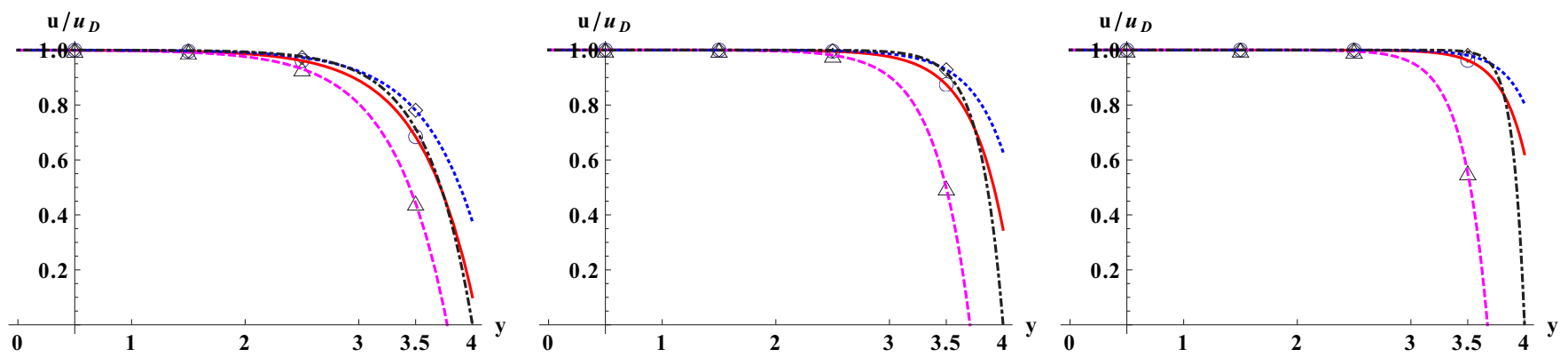

FIG. 15. (Color online) Similarly as in Fig. 7 but with the IBF.

In turn, Eqs. (26) and then Eq. (42b) are valid to apply $\delta_{i}=0$ in them, since they correspond to the effective discretization of the IBF scheme.

Remark 1. The IBF is equivalent to $\mathrm{BF}^{+}$via eigenvalue transform (52) applied to coefficients $A^{ \pm}$from Eq. (53). Accordingly, coefficients $\alpha^{ \pm}$in Eq. (57) coincide with Eq. (B13).

Remark 2. Vanishing of the viscosity correction in the diagonal channel is extended straightforwardly by following Ref. [29] and replacing $\left(\Lambda_{\star}-\frac{1}{4}\right)$ with $\frac{1}{2}\left(\Lambda_{\star}-\frac{1}{4}\right)$ in Eqs. (58). Although derived in the straight channel, the computations in random media will show that the IBF improvements are not limited to channel flow.

Figure 15 shows that the IBF vanishes the oscillations displayed by BF in Fig. 7 and behaves very similarly to the IFD in Fig. 6 for intermediate $\Lambda$, such as $\Lambda=\frac{3}{16}$. On the other hand, the IBF has very sharp profiles on the boundary or interface when $\Lambda \rightarrow 0$. The curvature coefficient $\alpha^{-}$in IBF is independent of the flow regime and is exactly the same as in Stokes flow. This last feature explains the improvment of the velocity continuity condition observable in Fig. 10 when $\Lambda$ lies in the basic (Stokes) $\Lambda$ interval. Typically, reduction of the interface velocity jump with IBF results in an improvment of permeability measurements, e.g., by a factor of 2 for $\Lambda=\frac{1}{8}$ in Fig. 10 .

When the permeability distribution is heterogeneous, the IBF applies with local values $B(\vec{r})$ in Eqs. (54), and hence with $B_{i}$ in multilayers. Figure 16 illustrates the evolution of two values, $\left\{\Lambda_{\star}^{(1)}, \Lambda_{\star}^{(2)}\right\}$ and $\left\{\alpha_{1}^{+}, \alpha_{2}^{+}\right\}$, towards $\sigma=\mathrm{Da}^{-1 / 2}$ [cf. Eq. (23)] in two periodic layers of permeability contrast $k_{2} / k_{1}=10^{3}$ for four input values of control parameter $\Lambda=$ $\left\{\frac{1}{100}, \frac{1}{12}, \frac{3}{16}, \frac{3}{8}\right\}$. When $\Lambda=\frac{3}{8}$, then $\Lambda_{\star}^{(1)}=\Lambda_{\star}^{(2)} \equiv \Lambda$ and $\alpha_{1}^{+}=$ $\alpha_{2}^{+} \equiv 1$ for all $\sigma$. Otherwise, in the intermediate zone, one observes that $\Lambda_{\star}^{(1)} \geqslant \Lambda_{\star}^{(2)}$ and $\alpha_{1}^{+} \leqslant \alpha_{2}^{+}$(with $\left\{\Lambda_{\star}^{(1)}, \alpha_{1}^{+}\right\}$in the less permeable layer). In the Stokes limit $\sigma \rightarrow 0, \Lambda_{\star}^{(i)}=\Lambda$ and $\alpha_{i}^{+}=1$. In the Darcy limit $\sigma \rightarrow \infty, \Lambda_{\star}^{(1)}$ and $\Lambda_{\star}^{(2)}$ take the limit value $\Lambda_{\star}^{(i)}=\frac{3}{8}$ while $\alpha_{i}^{+}=\frac{8 \Lambda}{3}$. In this limit, $\alpha_{i}^{ \pm} \rightarrow 0$ when $\Lambda \rightarrow 0$, i.e., the two interface velocity values in relations (36b) are approached improving for permeability.

However, since $\alpha_{i}^{+} \equiv 1$ for $\Lambda=\frac{3}{8}$ and this choice is quite inaccurate for interface and permeability measurements, we should conclude that the the curvature coefficient $\alpha_{i}^{-}$ dominates accuracy of these solutions, similarly to what happens in Stokes flow.

Further comparisons for IBF with the BF and FEM in stratified channels are found in Figs. 17-20. The solutions for "optimal" $\Lambda$ are presented in Figs. 21 and 22. Permeability predictions for three schemes are compared in Figs. 23-25 in two layers, followed by results in Figs. 26-31 for 1D and 2D random distributions.

\section{The "partial bounce back" gray schemes}

According to Ref. [49], gray schemes can be implemented in the common form:

$$
\begin{aligned}
f_{q}\left(\vec{r}+\vec{c}_{q}, t+1\right) & =\tilde{f}_{q}+k^{+} f_{q}+k^{-} f_{\bar{q}}+\tilde{k}^{+} \tilde{f}_{q}+\tilde{k}^{-} \tilde{f}_{\bar{q}}+F_{q}, \\
\tilde{f}_{q}(\vec{r}, t) & =f_{q}(\vec{r}, t)+g_{q}^{+}+g_{q}^{-}, \quad \text { with } \\
e_{q}^{-} & =t_{q}\left(\vec{J} \cdot \vec{c}_{q}\right), \\
\vec{J} & =\sum_{q=1}^{Q_{m}} f_{q} \vec{c}_{q}, \quad F_{q}=t_{q}\left(1-n_{s}\right)\left(\vec{F}^{p} \cdot \vec{c}_{q}\right) .
\end{aligned}
$$
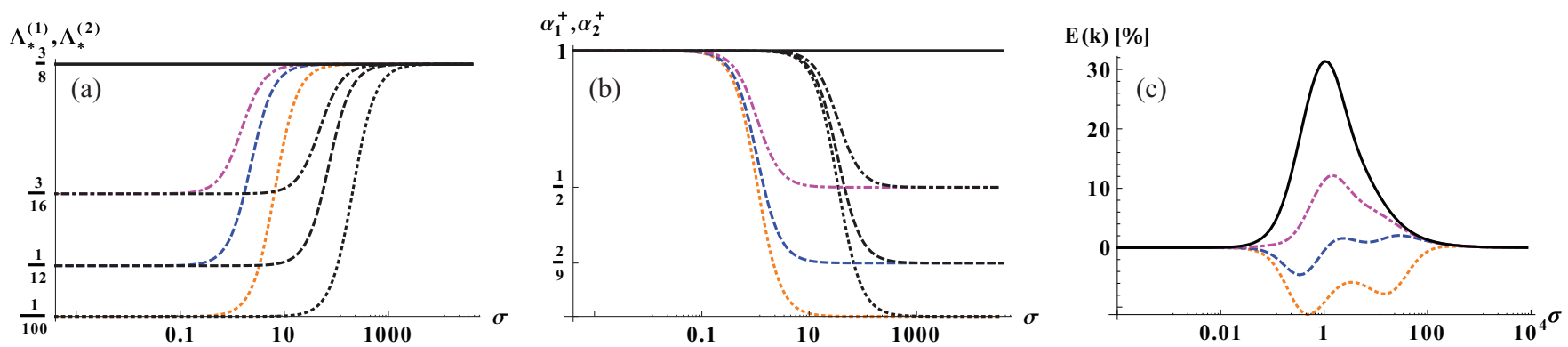

FIG. 16. (Color online) Two-layered periodic system of permeability contrast $k_{2} / k_{1}=10^{3}$ and $h_{1}=h_{2}=6$ by use of the IBF scheme. The left and middle diagrams plot for two layers (a) effective distributions $\left\{\Lambda_{\star}^{(1)}, \Lambda_{\star}^{(2)}\right\}$ from Eq. (55) and (b) coefficients $\left\{\alpha_{1}^{+}, \alpha_{2}^{+}\right\}$from Eq. (57), with light gray (color line) denoting the low-permeable layer where $\Lambda_{\star}^{(1)} \geqslant \Lambda_{\star}^{(2)}$ and $\alpha_{1}^{+} \leqslant \alpha_{2}^{+}$and the black lines the second layer. The results are plotted for $\Lambda=\left\{\frac{1}{100}, \frac{1}{12}, \frac{3}{16}, \frac{3}{8}\right\}$ (dot, dashed, dot-dashed, solid). The right plot shows the associated permeability error. 

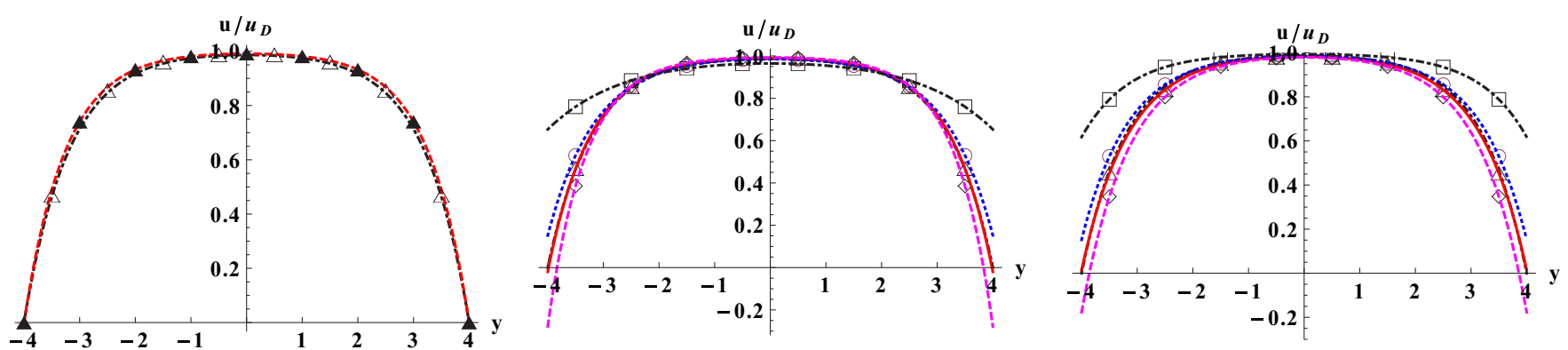

FIG. 17. (Color online) The Stokes-Brinkman profiles for $\sigma=10$ in a single porous channel of width $H=8$. Left diagram: Predicted FEM solution (A9) is plotted with the dashed (red) line; COMSOL solution with the black symbols; $\mathrm{BF}\left(\Lambda=\frac{1}{8}\right)$ solution (A3) with open symbols; the analytical solution is shown in dot-dashed (black). $\operatorname{The} \operatorname{BF}(\Lambda)$ and $\operatorname{IBF}(\Lambda)$ symbolic solutions are plotted in the middle and right diagrams for $\Lambda=\left\{\frac{1}{512}, \frac{3}{16}, \frac{3}{8}, 2\right\}$ (dashed,solid,dotted,dot-dashed). Accordingly, the symbols triangle, circle, lozenge, square mark the grid solution $U_{j}$. The analytical solution is very closely matched by $\Lambda=\frac{3}{16}$.

The coefficients $k^{+}$to $\tilde{k}^{-}$are related to the locally prescribed "solid" fraction $n_{s}$ as summarized in Table III for schemes called $\mathrm{GS}^{\star}[35,36], \mathrm{WBS}^{\star}$ [37], and $\mathrm{ZM}^{\star}$ [38]. All of them introduce momentum sink equal to $-2 n_{s} \vec{J}$. The redefinition of their macroscopic solution $\vec{J}=\rho_{0} \vec{u}$ with $\frac{1}{2} \vec{F}=\frac{1}{2}\left(\vec{F}^{p}-B_{f} \vec{J}\right)$ is imperative to obtain a constant Darcy velocity in series of blocks [49]. This has been realized in Ref. [37] by averaging the incoming and outgoing momentum. The three schemes then relate $\vec{j}$ to $\vec{J}$ exactly as in the BF model (7) and they have the common expression for local permeability value $k\left(n_{s}, v\right)$ with

$$
\begin{aligned}
\vec{J} & =\left(1-n_{s}\right)\left(\vec{J}+\frac{1}{2} \vec{F}^{p}\right)=\vec{J}+\frac{\left(F^{(p)}-B_{f} \vec{J}\right)}{2+B_{f}}, \\
B_{f} & =\frac{v}{k}=\frac{2 n_{s}}{1-n_{s}}, \quad k\left(n_{s}, v\right)=\frac{\left(1-n_{s}\right) v}{2 n_{s}} .
\end{aligned}
$$

Applying Eqs. (4) in Eq. (60), and keeping in mind that the sum of coefficients $k^{+}$to $\tilde{k}^{-}$is equal to zero in all schemes keeping mass conserved, the gray schemes are matched by the modified operator (50) with the entire forcing $\vec{F}=\vec{F}^{p}-B_{f} \vec{J}$ and coefficients $A^{ \pm}$gathered in Table III:

$$
\begin{aligned}
& A^{+}=-\frac{k^{+}+k^{-}+\tilde{k}^{+}+\tilde{k}^{-}}{s^{+}}+\left(\tilde{k}^{+}+\tilde{k}^{-}\right), \\
& A^{-}=-\frac{k^{+}-k^{-}+\tilde{k}^{+}-\tilde{k}^{-}}{s^{-}}+\left(\tilde{k}^{+}-\tilde{k}^{-}\right) .
\end{aligned}
$$

The modeled Brinkman channel equation retains form (33)(34b) with boundary or interface conditions (35) and (36). They should be read with $\delta=\delta_{\star}\left(A^{ \pm}\right)$given by Eq. (B9) and $\alpha^{ \pm}$given by Eq. (B11). All of them are reported in Table III. The specific solution for $\Lambda\left(B, \Lambda^{+}\right)$which vanishes $\delta_{\star}\left(A^{ \pm}\right)$ simply solves the linear equation $\delta_{\star}(\Lambda)=0$ (cf. Table 4 in Ref. [49]). Table III shows that, on top of $B$ and $\Lambda, \delta_{\star}\left(A^{ \pm}\right)$ and $\alpha^{ \pm}\left(A^{ \pm}\right)$nonlinearly depend on the kinematic viscosity $v$ via $\Lambda^{+}=3 v_{B}$. Their dimensionless velocity profiles $u_{x} / u_{D}$ then differs for two viscosity values, as illustrated in Fig. 14. This deficiency is smaller in the $\mathrm{WBS}^{\star}$ scheme, for these data at least. The $\mathrm{GS}^{\star}$ scheme has larger amplitude, negative value $\delta_{\star}\left(A^{ \pm}\right)$and numerically is much less stable than all others.

Thus, unlike the BF and IBF, these three gray schemes feature viscosity-dependent permeability solutions, even in the frame of the TRT model with fixed $\Lambda(\vec{r})$ distribution. It follows that these schemes reduce to $\mathrm{BF}$ via viscosity-dependent eigenvalue transforms, and vise versa, as given in Tables 5 and 6 in Ref. [49]. Indeed, with help of their free parameter $\Lambda$, the $\mathrm{GS}^{\star}$ and $\mathrm{ZM}^{\star}$ get solutions of the $\mathrm{BF}\left(s_{\star}^{-}\right)$by substituting their coefficients $A^{ \pm}$into Eq. (52) to get $s^{-}\left(s_{\star}^{-}\right)$. This way, they may directly get solutions of each other and IBF applying that following eigenvalue transform, relating any couple of two models (50):

$$
\left(1+A_{\star}^{+}\right) s_{\star}^{+}=\left(1+A^{+}\right) s^{+}, \quad\left(1+A_{\star}^{-}\right) s_{\star}^{-}=\left(1+A^{-}\right) s^{-} .
$$
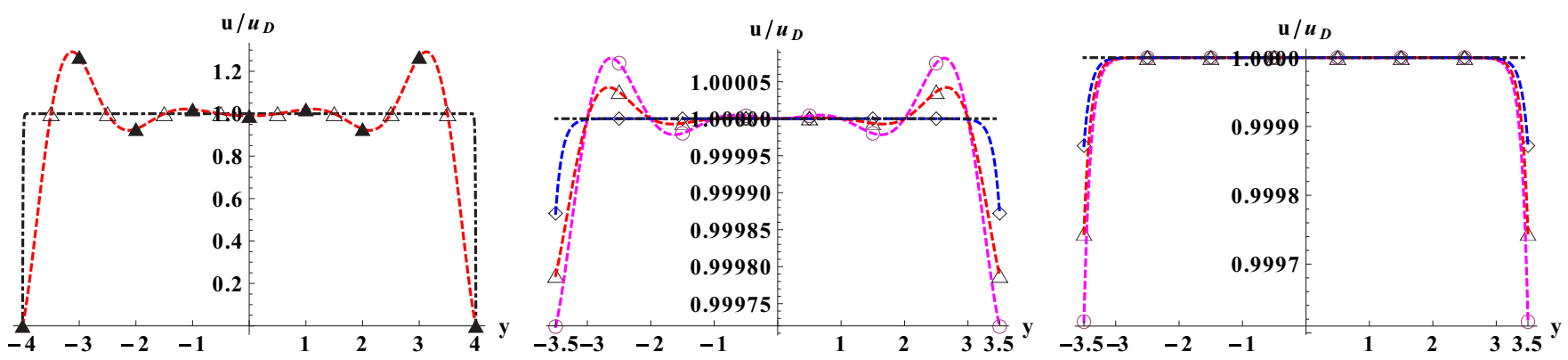

FIG. 18. (Color online) The exact solution (25) is plotted by the dot-dashed (black) line for $\sigma=10^{3}$ in the single channel of width $H=8$. Left diagram: FEM solution (A9) is plotted with the dashed (red) line, COMSOL solution with the black symbols, and $\mathrm{BF}\left(\Lambda=\frac{1}{8}\right)$ with open symbols. In middle and right diagrams, the grid-node solution is zoomed for $\Lambda=\left\{\frac{1}{8}, \frac{3}{16}, \frac{3}{8}\right\}$ (circles, triangles, lozenges), with BF and IBF, respectively. 

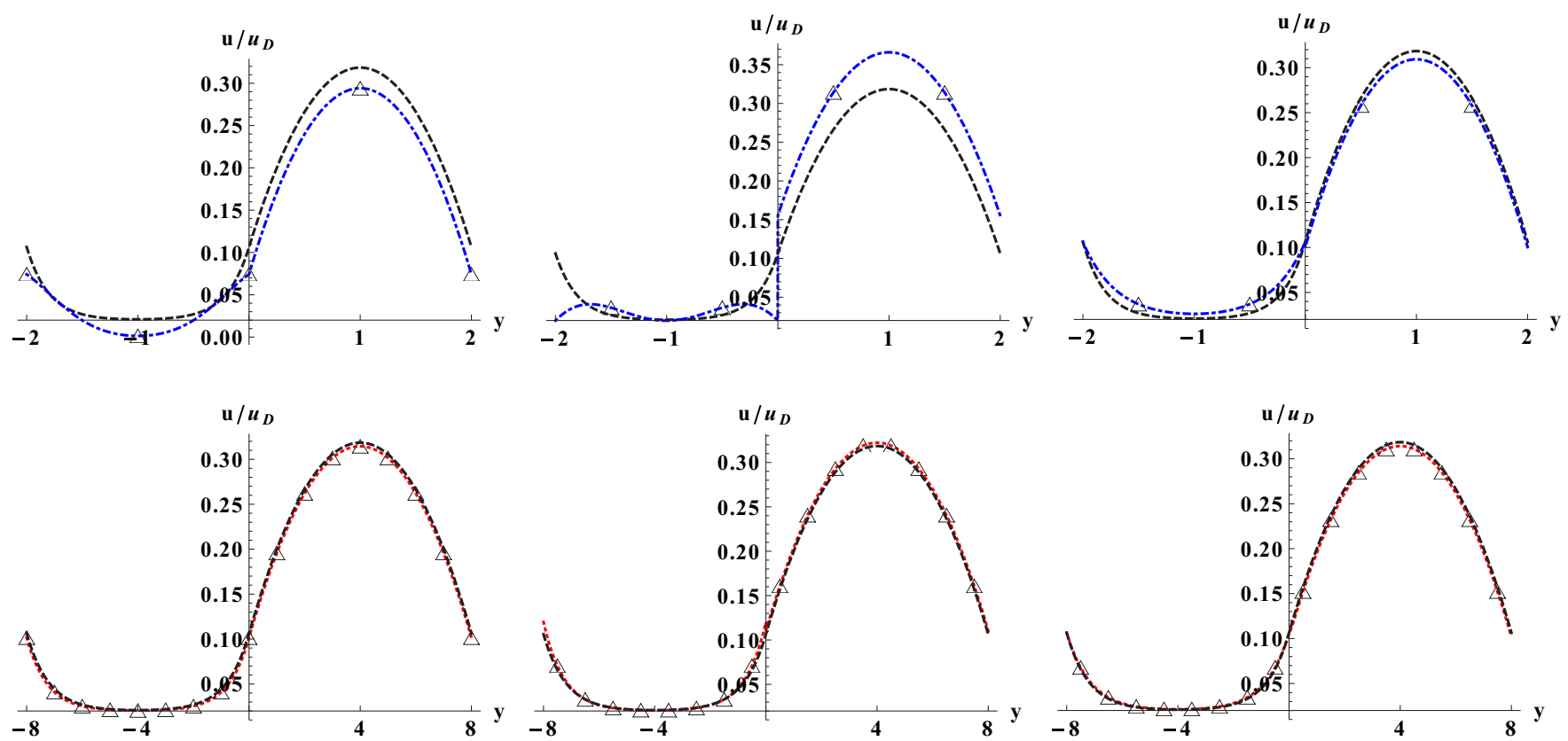

FIG. 19. (Color online) The same configuration as in Fig. 9: $\operatorname{FEM}, \operatorname{BF}\left(\Lambda=\frac{1}{8}\right)$, and $\operatorname{IBF}\left(\Lambda=\frac{1}{8}\right)$, from left to right, with $h_{i}=H / 2=2$ in the top row and $h_{i}=8$ in the bottom.

The WBS* scheme will also obtain solutions of BF, IBF, $\mathrm{GS}^{*}$, and $\mathrm{ZM}^{*}$ but transforming its two relaxation rates with Eq. (63).

In summary, the gray schemes have shown that, with a naive microscopic modification (50) of a population update, the solutions may violate the dimensional groups of the modeled equation. This is easily observable in $v_{B}$ dependency for equivalent eigenfunctions $\Lambda_{\star}^{+}$in the standard population update when a viscosity-independent control parameter $\Lambda$ is set. Unlike the gray schemes, the IBF remains consistent in such parameter configuration due to its linear dependency $\Lambda_{\star}^{+}\left(v_{B}\right)$.

\section{THE FEM BRINKMAN SCHEME IN STRATIFIED CHANNELS}

We assume constant pressure distribution and constant density $\rho_{0}$, the flow being driven along the channel axis $x$ by the constant external force $F_{x}^{(p)}$. The material properties $k_{e}^{(i)}$ and $\phi_{i}$ are piecewise constant per layer. The grid is uniform with space step $\delta_{y}$ along the $y$ axis. Velocity and stress-continuity conditions are prescribed on the interface $Y_{i}$ as follows:

$$
\left.u_{x}\right|_{Y_{i}-}=\left.u_{x}\right|_{Y_{i}+},\left.\quad v_{B}^{(i)} \partial_{y} u_{x}\right|_{Y_{i}-}=\left.v_{B}^{(i)} \partial_{y} u_{x}\right|_{Y_{i}+}
$$

The standard Galerkin formulation is applied to discretize Eq. (1). The velocity is discretized on the $N_{e}+1$ vertexes $y_{j}$ of $N_{e}$ elements: $u_{x}(y)=\sum_{j=1}^{N_{e}+1} u_{j}^{(i)} \Phi_{j}$. A piecewise-continuous global interpolation function $\Phi_{j}(y)$ is defined featuring the linear shape functions: $\Phi_{j}(y)=\psi_{2}(y), y \in\left[y_{j-1}, y_{j}\right], \Phi_{j}(y)=\psi_{1}(y), y \in\left[y_{j}, y_{j+1}\right]$, $\psi_{1}(\xi)=(1+\xi) / 2, \quad \psi_{2}(\xi)=(1-\xi) / 2$, with $\xi \in[-1,1]$ in local coordinates. The discretization of the drag forcing considers the interface vertex $y_{j}=Y_{i}$ to be shared by two elements $\left[y_{j-1}, y_{j}\right]$ and $\left[y_{j}, y_{j+1}\right]$. Hence, inside each element, the drag force is approximated as $\quad F^{r(i)}(y)=-F_{e}^{(i)} \rho_{0} u_{x}(y), \quad F_{e}^{(i)}=\frac{v}{k_{e}^{(i)}}$. Multiplying Eq. (1) by $\Phi_{j}$ and integrating over the domain yields $\int_{y_{j-1}}^{y_{j}} F^{r(i)}(y) \Phi_{j}(y) d y=-F_{e}^{(i)} \rho_{0}\left(u_{j-1}^{(i)} b_{j-1}+u_{j}^{(i)} b_{j}\right) \delta_{y}$, $\int_{y_{j}}^{y_{j+1}} F^{r(i)}(y) \Phi_{j}(y) d y=-F_{e}^{(i+1)} \rho_{0}\left(u_{j}^{(i)} b_{j}+u_{j+1}^{(i+1)} b_{j+1}\right) \delta_{y}$,
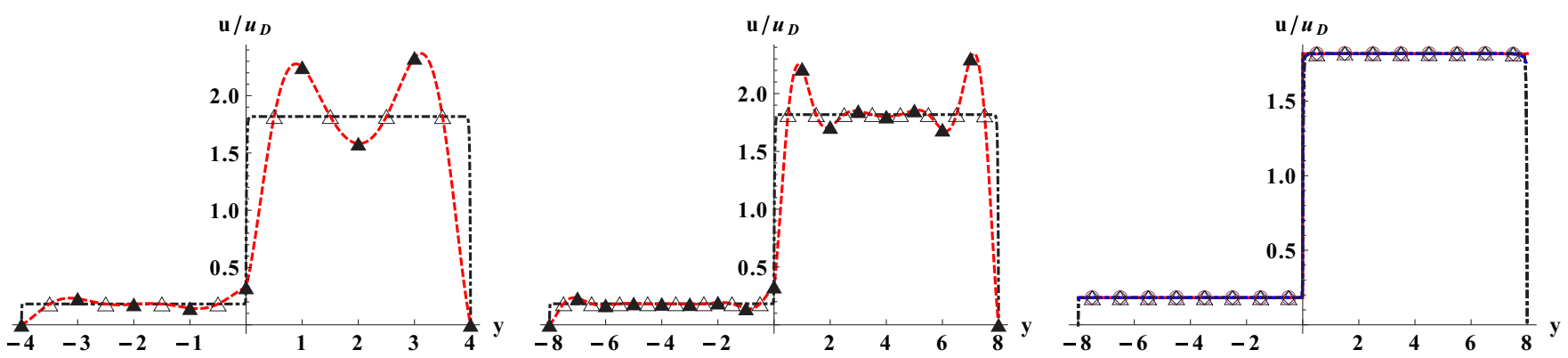

FIG. 20. (Color online) Exact solution in two-layered bounded channel is plotted with a dot-dashed line (black), predicted FEM with a dashed (red) line, COMSOL with filled (black) symbols, and $\mathrm{BF}\left(\Lambda=\frac{1}{8}\right)$ with open symbols (left and middle diagrams). Left diagram: $k_{2} / k_{1}=10$, $\sigma=10^{3}, H=8$. Middle diagram: $k_{2} / k_{1}=10, \sigma=10^{3}, H=16$. Right diagram: BF with $\Lambda=\left\{\frac{1}{8}, \frac{3}{16}, \frac{3}{8}\right\}$. 

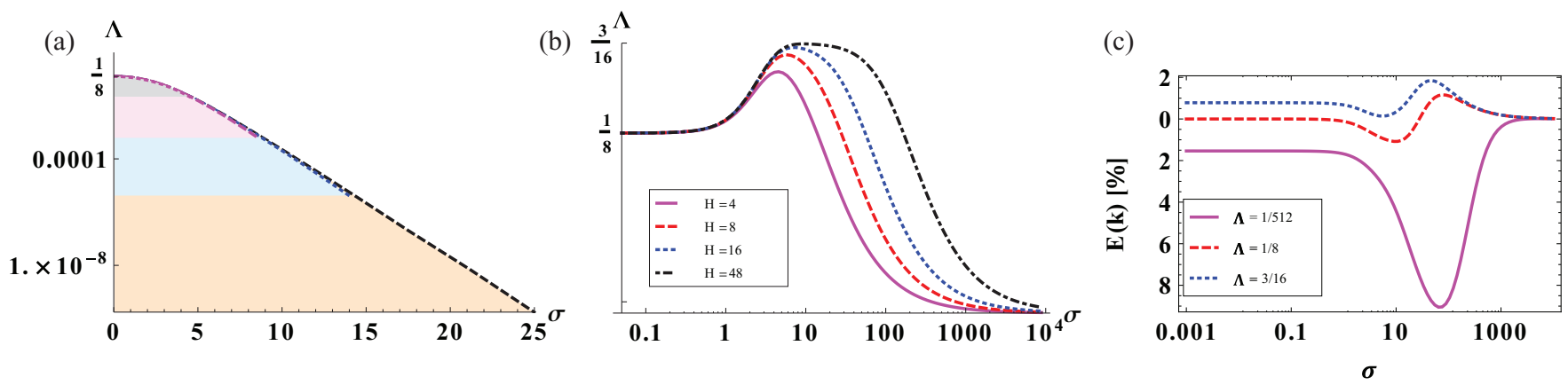

FIG. 21. (Color online) The left and middle diagrams plot the solution for $\Lambda(\sigma)$ where $E(k)=0$ in the single porous channel $H=$ $\{4,8,16,48\}$ : (a) BF, $\sigma<2 H$ (shaded with different color for different $H$ ); (b) IBF, $\forall \sigma$. Right diagram: plot of $E(k)$ in IBF for $H=8$ when $\Lambda=\left\{\frac{1}{512}, \frac{1}{8}, \frac{3}{16}\right\}$ (solid, dashed, dotted).

with $\quad b_{j-1}=b_{j+1}=\frac{1}{2} \int_{-1}^{1} \psi_{1}(\xi) \psi_{2}(\xi) d \xi=\frac{1}{6}, \quad b_{j}=$ $\frac{1}{2} \int_{-1}^{1} \psi_{1}(\xi) \psi_{1}(\xi) d \xi=\frac{1}{2} \int_{-1}^{1} \psi_{2}(\xi) \psi_{2}(\xi) d \xi=\frac{1}{3}$. The variational formulation of the Brinkman equation (1) takes the following discretized form in vertex $j$ :

$$
\begin{aligned}
& \frac{\delta_{y}}{2}\left[-F_{x}^{(p)}+\frac{F_{e}^{(i)}}{3} \rho_{0}\left(2 u_{j}^{(i)}+u_{j-1}^{(i)}\right)\right] \\
+ & \frac{\delta_{y}}{2}\left[-F_{x}^{(p)}+\frac{F_{e}^{(i+1)}}{3} \rho_{0}\left(2 u_{j}^{(i+1)}+u_{j+1}^{(i+1)}\right)\right] \\
= & v_{B}^{(i+1)} \rho_{0} \frac{u_{j+1}^{(i+1)}-u_{j}^{(i+1)}}{\delta_{y}}-v_{B}^{(i)} \rho_{0} \frac{u_{j}^{(i)}-u_{j-1}^{(i)}}{\delta_{y}}, \\
& \text { with } \quad F_{e}^{(i)}=\frac{v}{k_{e}^{(i)}} .
\end{aligned}
$$

The equivalent form reads

$$
\begin{aligned}
\frac{\delta_{y}}{2}( & \left.-F_{x}^{(p)}+F_{e}^{(i)} \rho_{0} u_{j}^{(i)}\right)+\frac{\delta_{y}}{2}\left(-F_{x}^{(p)}+F_{e}^{(i+1)} \rho_{0} u_{j}^{(i+1)}\right) \\
= & \left(v_{B}^{(i+1)}-\frac{F_{e}^{(i+1)} \delta_{y}^{2}}{6}\right) \rho_{0} \frac{u_{j+1}^{(i+1)}-u_{j}^{(i+1)}}{\delta_{y}} \\
& -\left(v_{B}^{(i)}-\frac{F_{e}^{(i)} \delta_{y}^{2}}{6}\right) \rho_{0} \frac{u_{j}^{(i)}-u_{j-1}^{(i)}}{\delta_{y}}
\end{aligned}
$$

Inside each layer system obeys:

$$
\begin{aligned}
& -F_{x}^{(p)}+F_{e}^{(i)} \rho_{0} u_{j}^{(i)} \\
& =v_{B}^{(i)}\left(1-\frac{1}{6} \frac{F_{e}^{(i)} \delta_{y}^{2}}{v_{B}^{(i)}}\right) \rho_{0} \frac{u_{j+1}^{(i)}-2 u_{j}^{(i)}+u_{j-1}^{(i)}}{\delta_{y}^{2}} .
\end{aligned}
$$

Assuming that the velocity $u_{j}^{(i)}$ satisfies Eq. (67) for its virtual continuation to node $j+1$, and then replacing
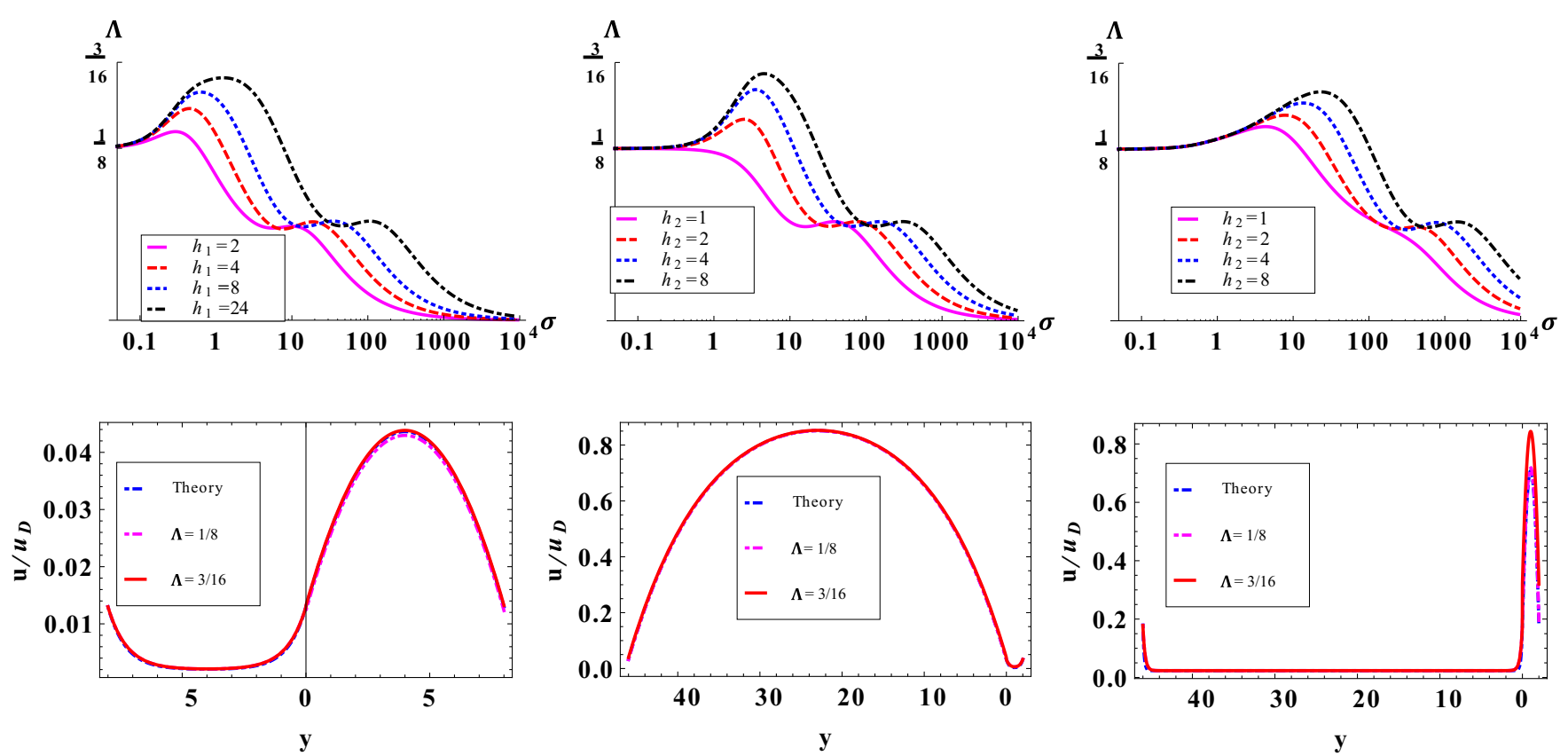

FIG. 22. (Color online) Top row: Predicted solution $\Lambda(\sigma)$ where IBF yields $E(k)=0$ in three two-layered systems illustrated in bottom row for $\sigma=\{1,5,50\}$ from the left to the right. Left diagrams: $R_{h}=h_{1} / h_{2}=1, R_{k}=k_{2} / k_{1}=10^{3}$. Middle diagrams: $R_{h}=h_{1} / h_{2}=23$, $R_{k}=k_{1} / k_{2}=10^{3}$. Right diagrams: $R_{h}=h_{1} / h_{2}=23, R_{k}=k_{2} / k_{1}=10^{3}$. 


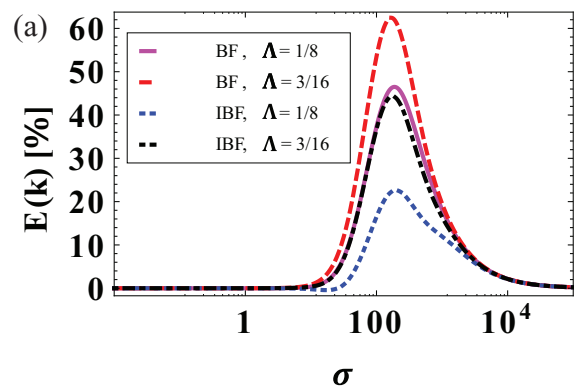

(c)

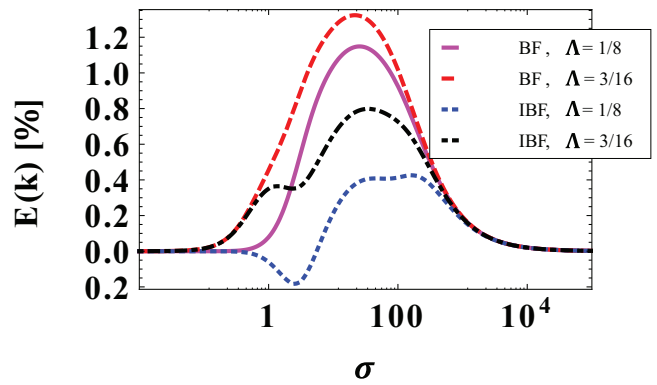

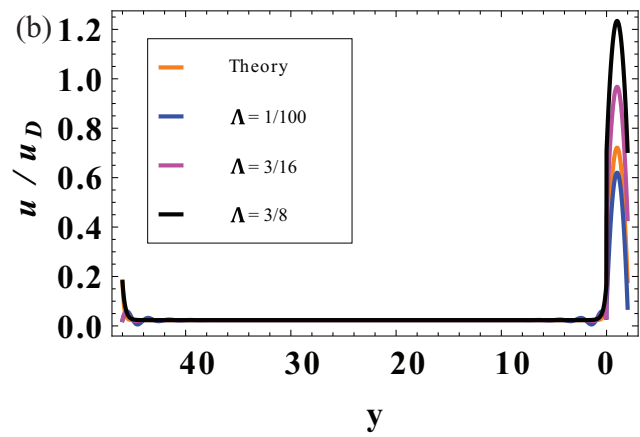

(d)

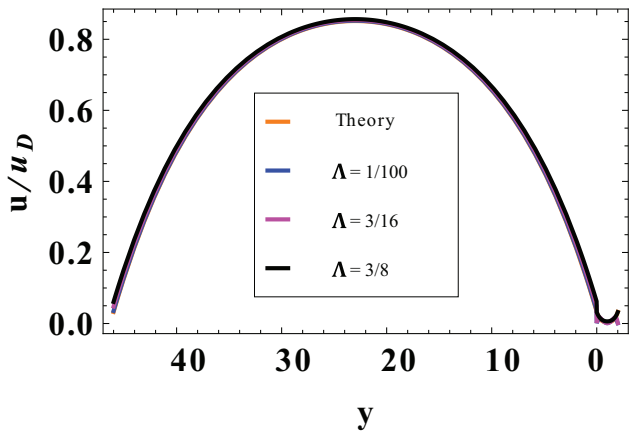

FIG. 23. (Color online) The two-layered asymmetric periodic systems of width $H=48$ with the BF and IBF when $r_{k}^{2}=k_{1} / k_{2}=10^{-3}$ [(a) and (b)] and $r_{k}^{2}=k_{1} / k_{2}=10^{3}$ [(c) and (d)]; the open layer is resolved with 2 [(a) and (b)] and 46 [(c) and (d)] space steps, respectively. [(a) and (c) $] \operatorname{BF}\left(\Lambda=\frac{3}{16}\right), \operatorname{BF}\left(\Lambda=\frac{1}{8}\right), \operatorname{IBF}\left(\Lambda=\frac{3}{16}\right), \operatorname{IBF}\left(\Lambda=\frac{1}{8}\right)$, from the topmost line. [(b) and (d)] The profiles are plotted with the $\mathrm{BF}$ for $\sigma=50$ (b) and $\sigma=5$ (d). Compare with the IBF profiles in Fig. 22.

$\frac{\delta_{y}}{2}\left(-F_{x}^{(p, i)}+F_{e}^{(i)} \rho_{0} u_{j}^{(i)}\right) \quad$ in Eq. (66) by $\quad v_{B}^{(i)}(1-$ $\left.\frac{1}{6} \frac{F_{e}^{(i)} \delta_{y}^{2}}{v_{B}^{(i)}}\right) \rho_{0} \frac{u_{j+1}^{(i)}-2 u_{j}^{(i)}+u_{j-1}^{(i)}}{2 \delta_{y}}$, Eq. (66) reads on the interface:

$$
\begin{gathered}
v_{B}^{(i+1)}\left(1-\frac{1}{6} \frac{F_{e}^{(i+1)} \delta_{y}^{2}}{v_{B}^{(i+1)}}\right) \rho_{0} \frac{u_{j+1}^{(i+1)}-u_{j-1}^{(i+1)}}{2 \delta_{y}} \\
=v_{B}^{(i)}\left(1-\frac{1}{6} \frac{F_{e}^{(i)} \delta_{y}^{2}}{v_{B}^{(i)}}\right) \rho_{0} \frac{u_{j+1}^{(i)}-u_{j-1}^{(i)}}{2 \delta_{y}} .
\end{gathered}
$$

This corresponds to the interface stress continuity conditions in the following form:

$\left.\mathrm{FEM}: \| v_{i} \bar{\Delta}_{y} u_{j}^{(i)}\right) \|_{y_{j}=Y_{i}}=0, \quad \bar{\Delta}_{y} u_{j}^{(i)}=\left(u_{j+1}^{(i)}-u_{j-1}^{(i)}\right) / 2$,

$$
v_{i}=\left(1+\delta_{i}\right) v_{B}^{(i)} \text {. }
$$
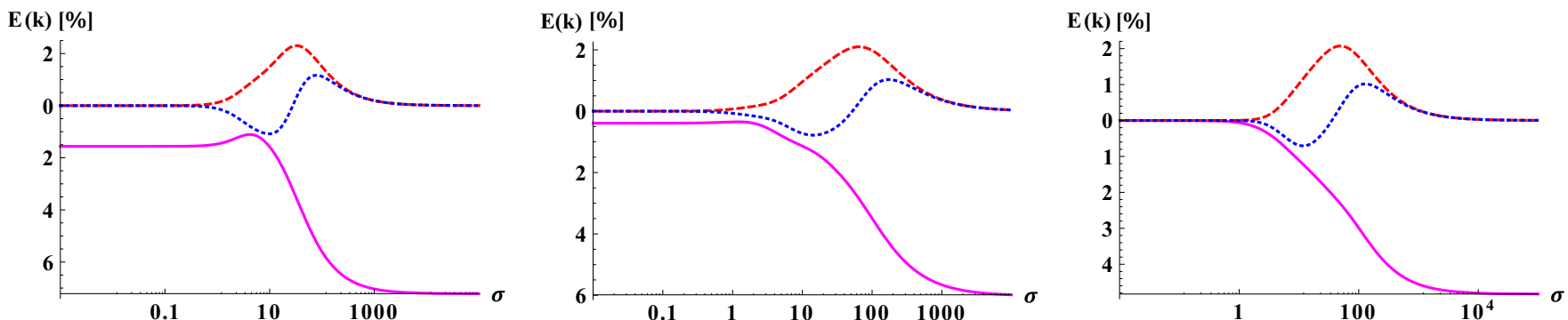

FIG. 24. (Color online) Predicted permeability error $E(k)$ towards $\sigma$ is compared for $\mathrm{BF}\left(\Lambda=\frac{1}{8}\right)$ [dashed (red)], $\operatorname{IBF}\left(\Lambda=\frac{1}{8}\right)[\operatorname{dotted}(\mathrm{blue})]$, and FEM [solid (magenta)] in a single channel of width $H=8$ in the left diagram and for permeability contrast $R_{k}=10$ in two-equal-layered channels of width $H=16$, the bounded in the middle and the periodic in the right. 

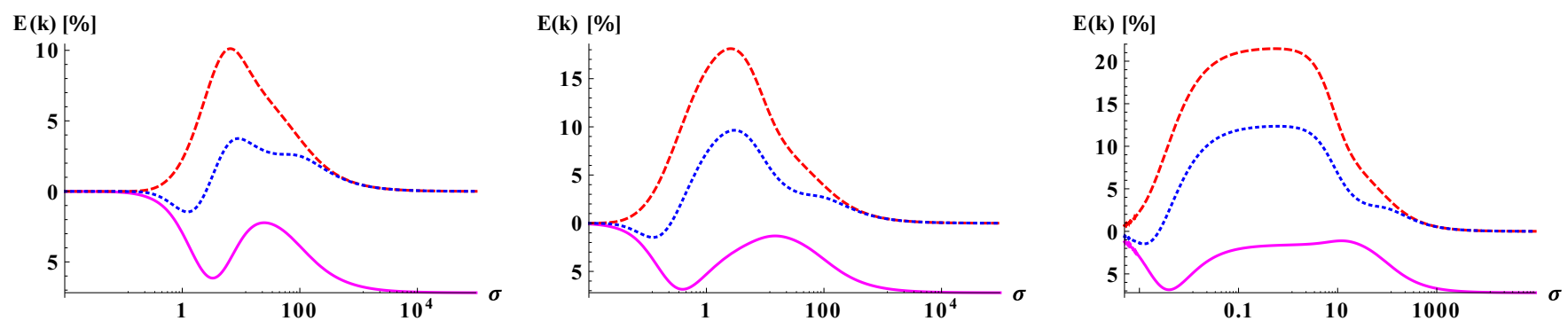

FIG. 25. (Color online) Predicted permeability error in periodic system of two equal layers of a width of eight grid cells with permeability contrast $R_{k}=\left\{10^{3}, 3 \times 10^{5}, 10^{9}\right\}$ for BF [dashed (red)], IBF [dotted (blue)], and FEM [solid (magenta)].
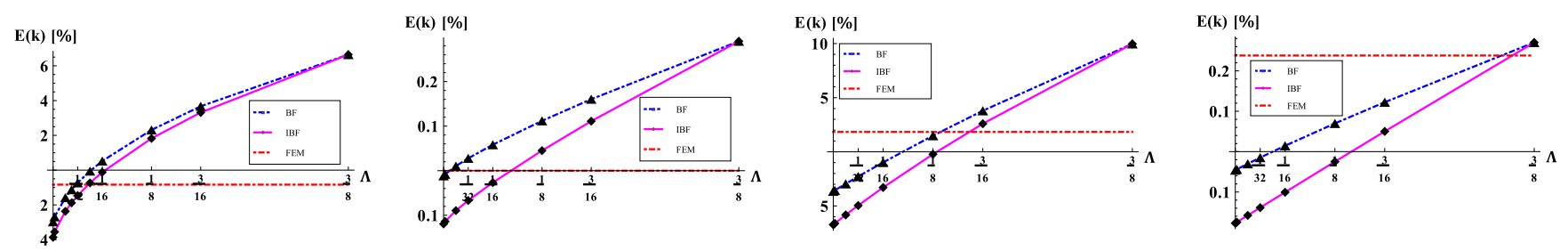

FIG. 26. (Color online) This and three next figures: In the two first diagrams $E(k)$ is plotted versus $\Lambda$ for a 2D Cauchy distribution in a $48 h^{2}$ box with $h=1$ and $h=8$. In two last diagrams, multilayered (ML1) simulations in 48 layers are displayed for one and eight grid cells per layer. The symbols include $\Lambda=\left\{10^{-6}, \frac{1}{512}, \frac{1}{64}, \frac{1}{32}, \frac{1}{16}, \frac{1}{8}, \frac{3}{16}, \frac{3}{8}\right\}$. The BF (dot-dashed blue line with triangles) and IBF (solid magenta line with lozenges). The FEM is independent of $\Lambda$ (dot-dashed red lines). This figure shows the Stokes-Brinkman zone $\sigma=15.2$ where $R_{k}=\frac{k_{B}}{k_{D}} \approx 0.34$ (2D) and $R_{k} \approx 0.09$ (ML1).
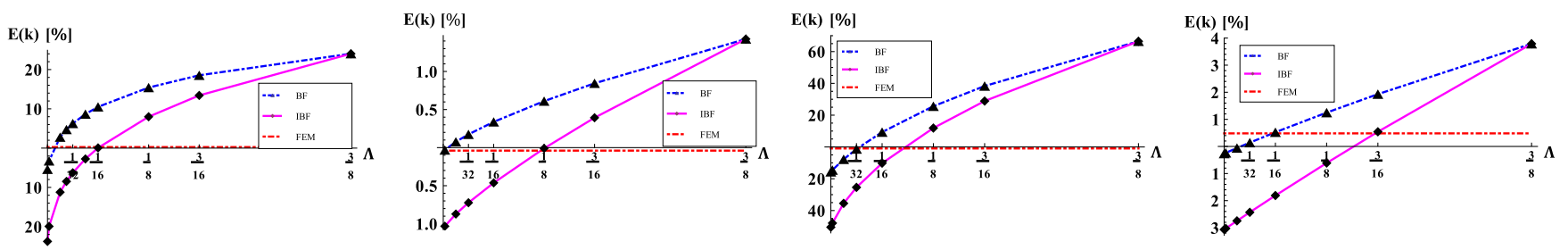

FIG. 27. (Color online) This figure shows the Brinkman zone $\sigma=152, R_{k}=\frac{k_{B}}{k_{D}} \approx 0.68$ (2D), and $R_{k} \approx 0.23$ (ML1).
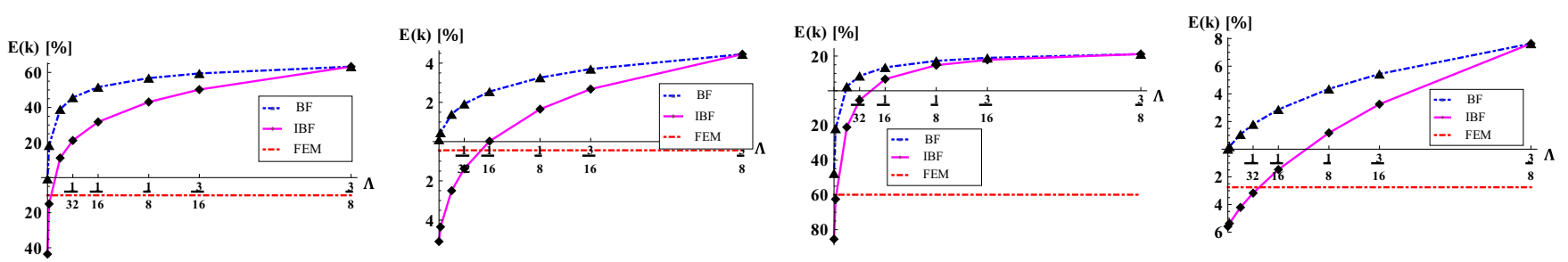

FIG. 28. (Color online) This figure shows the Darcy-Brinkman zone $\sigma=1520, R_{B}=\frac{k_{B}}{k_{D}} \approx 0.92$ (2D), and $R_{B} \approx 0.8$ (ML1).
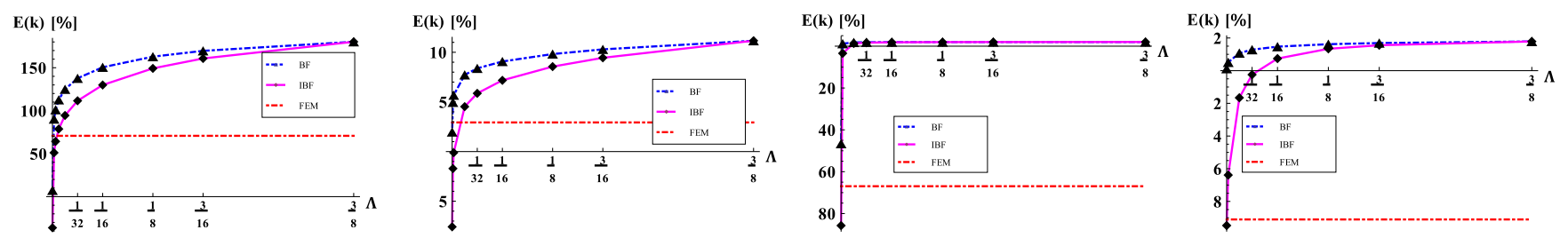

FIG. 29. (Color online) This figure shows the Darcy zone, $\sigma=\mathrm{Da}^{-1 / 2}=15200, R_{B}=\frac{k_{B}}{k_{D}} \approx 0.97$ (2D), and $R_{B} \approx 0.98$ (ML1). 

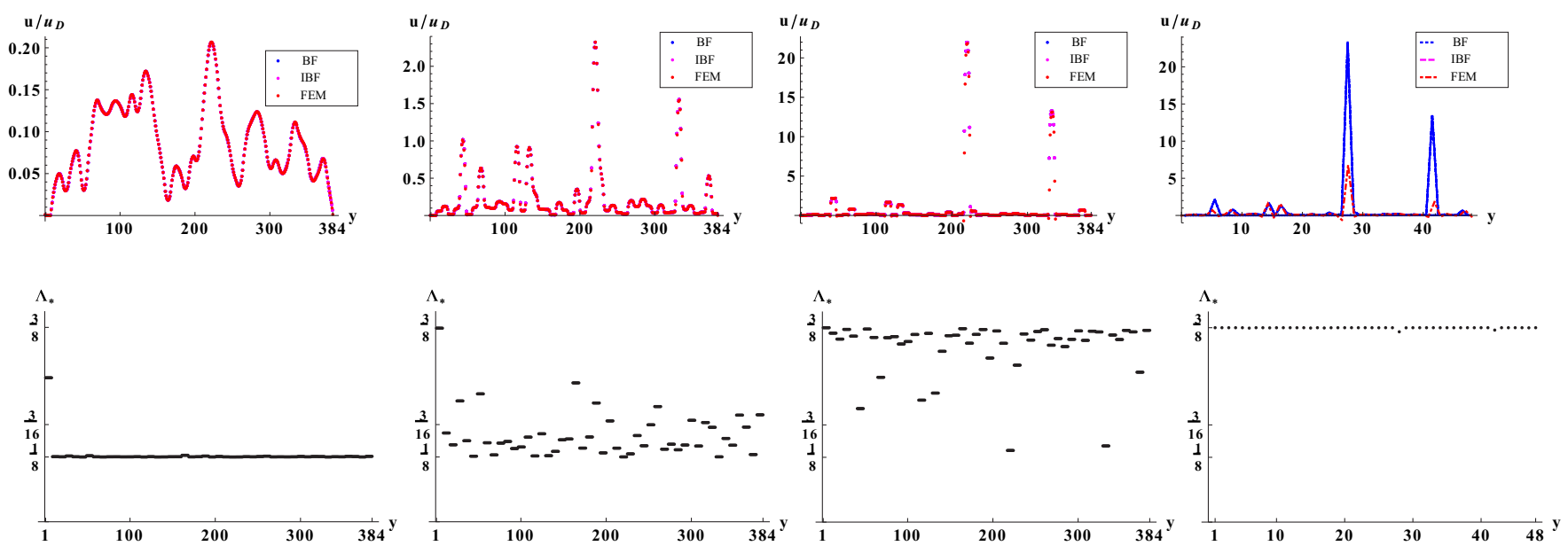

FIG. 30. (Color online) Top row: Velocity distribution in ML1 with BF (blue), IBF (magenta), and FEM (red), with $\sigma=\mathrm{Da}^{-1 / 2}=$ $\{15.2,152,1520,15200\}$ from left to right. In the fourth diagram, BF and IBF almost coincide, and FEM is shown in a dot-dashed line. Grid refining $h=8$ except for the last diagram, where $h=1$. Bottom row: Corresponding effective distribution $\Lambda_{\star}$ in IBF given by Eq. (55). The $\mathrm{BF} / \mathrm{IBF}$ are applied with $\Lambda=\frac{1}{8}$ in the three first cases and $\Lambda=\frac{1}{64}$ in the last diagram where $\Lambda_{\star} \rightarrow \frac{3}{8}$ when $\sigma \rightarrow \infty$.

The solution is given by Eqs. (34) with:

$$
\begin{aligned}
\text { FEM }: \delta_{i}=-\frac{B_{i}}{6}=\left.\delta_{i}\left(B_{i}, \Lambda_{i}=\frac{1}{8}\right)\right|_{\mathrm{BF}}, \\
r_{i}=\frac{1 \pm \sqrt{3} \sqrt{\frac{B_{i}}{12+B_{i}}}}{1 \mp \sqrt{3} \sqrt{\frac{B_{i}}{12+B_{i}}}} .
\end{aligned}
$$

Thereby, the BF has this correction for particular value $\Lambda_{i}=\frac{1}{8}$. The symbolic solutions are constructed similarly as in BF prescribing Eqs. (34) with Eq. (71), along with Eq. (69) on the interfaces. The discretized system is determined by Eqs. (A9) and (A10). In bounded channels, velocity is set equal to zero in boundary points. The FEM symbolic solutions are found in quasiexact agreement with the COMSOL code profiles applying the relative residual stopping criteria (see in Sec. VIC). In periodic system, $u_{j}^{(i)}$ is set the same in first and last vertexes. Figure 17 shows the Stokes-Brinkman solution for $\sigma=10$ when $v_{i}>0$ with the three schemes. The FEM agrees well with the analytical solution, and the accuracy of BF and IBF depends on $\Lambda: \operatorname{IBF}\left(\Lambda=\frac{3}{16}\right)$ behaves the best among the displayed schemes. The large values $\Lambda>\frac{3}{16}$ overestimate the velocity profile.

Figure 18 addresses the Darcy regime for $\sigma=10^{3}$. The left diagram plots $\mathrm{FEM}$ and $\operatorname{BF}\left(\Lambda=\frac{1}{8}\right)$ : The two schemes have the same $\delta=-\frac{B}{6}$ predicted by Eq. (71). Since $B=\frac{10^{6}}{64} \gg$ $6, v^{\text {num }}<0$, the two schemes are expected to oscillate. The results display strong oscillations with the FEM, while $U_{j}$ lies on the Darcy flat solution branch for BF. The two next diagrams zoom into the $\mathrm{BF}$ and IBF solutions in grid nodes. Indeed, the BF displays the oscillation near the boundary for $\Lambda=\frac{1}{8}$. Yet their amplitude is about $10^{4}$ smaller than that from FEM. As expected, the IBF has no oscillations; when $\Lambda=\frac{3}{8}$, the $\mathrm{BF}$ and IBF coincide and their solution is monotonous.

Figure 19 addresses the same configurations as in Fig. 9 but with $\operatorname{FEM}, \operatorname{BF}\left(\Lambda=\frac{1}{8}\right)$, and $\operatorname{IBF}\left(\Lambda=\frac{1}{8}\right)$. It shows that oscillations appear in the low-permeability layer with FEM and $\operatorname{BF}\left(\Lambda=\frac{1}{8}\right)$ on the coarse grid where $1+\delta_{1}=1-B_{1} / 6<0$. Furthermore, the FEM solution is continuous on the interface but the velocity becomes negative in the low-permeability layer. The oscillations vanish with the IBF. All solutions improve noticeably for eight points per layer. The behavior is very similar in periodic and bounded channels (data is not shown) for the same parameter choice.

Figure 20 shows that in the Darcy-Brinkman regime in a bounded channel, FEM solutions display strong interface oscillation, on top of the boundary oscillations, similarly to that shown for single channel in Fig. 17. It is interesting to note that its relative amplitude $u_{x}^{(i)} / u_{D}$ does not improve by increasing resolution. At the same time, the grid-node velocity is located on the two Darcy branches with the BF/IBF, where $\mathrm{BF}$ has relatively small oscillations similar to what we have observed in a single channel in Fig. 17.

In summary, the FEM has the same bulk correction as the BF with $\Lambda_{i}=\frac{1}{8}$, meaning that their effective bulk equations
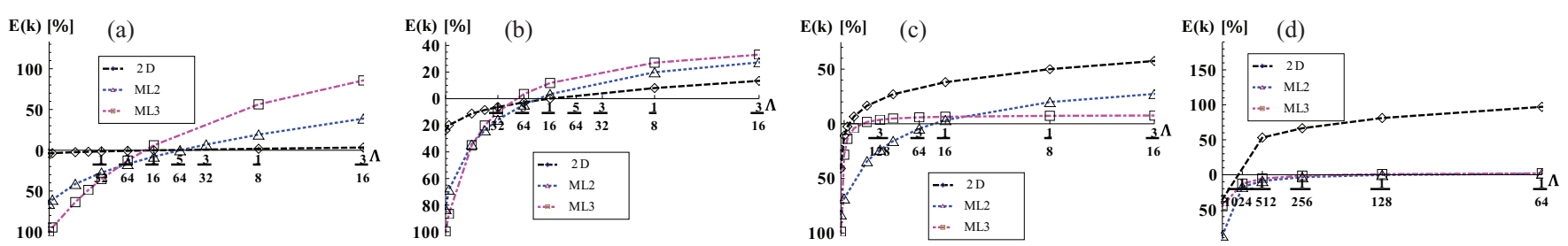

FIG. 31. (Color online) This figure plots $E(k)$ towards $\Lambda$ for three regimes (a)-(d): $\frac{k_{B}}{k_{D}} \approx\{0.3362,0.6796,0.925,0.97\}$. The random 2D (black lozenge), multilayers ML2 (blue triangles), and ML3 (magenta squares) are plotted together. The data are in Table IV. 
are the same. The only difference refers to the location of the discretization points with respect to boundary or interface. In turn, the boundary and interface conditions differ in two schemes. In the Stokes-Brinkman and Brinkman regimes the choice of $\Lambda$ is important for BF scheme in view of the bulk and boundary accuracy. The bulk profiles are smoother with the IBF, but $\Lambda$ still controls the boundary, more in the spirit of TRT Stokes scheme. In the Darcy-Brinkman and Darcy regimes, the bounce-back produces sufficiently accurate velocity in the grid nodes themselves, without any oscillations with the IBF, but it does not accommodate symbolic continuation of the profiles to zero boundary value, in opposition to FEM, which by enforcing this leads to strong oscillations. A similar situation takes place on the interface where enforcing the exact continuity conditions between Darcy profiles, FEM causes interface oscillations. Again, this is opposed to LBM where neighboring velocity branches stay "discontinuous," accommodated internally by an implicit interface layer. Nevertheless, the implicit coupling may produce large interface slip-velocity jumps even in the Stokes-Brinkman regime, especially visible with the $\mathrm{BF}$ scheme unless $\Lambda$ is reduced, but then the $\mathrm{BF}$ oscillates. The IBF noticeably improves this interface discrepancy for intermediate $\Lambda$ without any oscillations. All in all, this suggests that the accuracy of the permeability measurements will depend on the scheme and on $\Lambda$ with the $\mathrm{BF} / \mathrm{IBF}$ and that this last dependency will differentiate according to the Brinkman regime. This is further explored in the next section.

\section{THE PERMEABILITY}

This section begins with the symbolic predictions on the accuracy of permeability measurements for BF, IBF, or FEM in single and two-layered channels. We recall that by construction, the FEM supports linearity of Brinkman equation with respect to the kinematic viscosity $v$. This way, the derived permeability $k_{B}$ is independent of the viscosity values when the steady state is reached. The BF and IBF assure viscosity-independent permeability $k_{B}$ provided only that the distribution $\Lambda_{i}(\vec{r})$ is kept fixed when $\Lambda_{i}^{+}$is altered with $v$. The symbolic solutions will reveal that the optimal solution for $\Lambda$, where the numerical and predicted values coincide, strongly depends on the flow regime. Permeability measurements in a two-dimensional random Cauchy distribution, and its multilayered subdistributions, are then compared. These studies lead to a heuristic algorithm aimed at predicting the optimal $\Lambda$ in multidimensions from their multilayered counterparts. Another heuristic algorithm allows us to maintain a similar convergence to steady state in LBM through the different regimes and space resolutions.

In this section, all simulations are driven by the external constant forcing $F_{x}^{(p)} \approx 10^{-6}$ and $v_{B}^{(i)}=v\left[f_{i}(\phi)=1\right.$ in Eq. (1)]. The flow regime is characterized in terms of $\sigma=\mathrm{Da}^{-1 / 2}$ : The averaged Darcy number $\mathrm{Da}=k_{D} / H^{2}$ is computed with the predicted Darcy permeability value $k_{D}$. In parallel, the effective regime is characterized in terms of $R_{B}=k_{B} / k_{D}$ on the computed solution $k_{B}$. The permeability contrast $R_{k}=k^{\max } / k^{\min }$ and the aspect ratio $R_{h}=h^{\max } / h^{\min }$ (in two layers) complete the description.

\section{A. Single and two-layered systems}

We first examine the permeability error $E(k)$ of the measured permeability $k^{\text {num }}$ against the predicted value $k_{B}$ in single and two-layered systems:

$$
E(k)=\frac{k^{\text {num }}}{k_{B}}-1, \quad k^{\text {num }}=\frac{\left\langle u_{j}\right\rangle \rho_{0} v}{F_{x}^{(p)}} .
$$

In LBM, $\left\langle u_{j}\right\rangle$ is computed from the arithmetical average of the cell-centered grid velocity values, while the "trapezoidalrule" is applied for the vertex-centered grid in FEM. Reference solution $k_{B}$ is obtained from the integration of the exact profile in multilayers and from the finer-grid FEM solution in random media.

Remark The difference between the summation and integration explains $E(k) \neq 0$ for $\Lambda=\frac{3}{16}$ on the exact Poiseuille profile (see the caption to Fig. 10). The exact permeability value $k^{\text {num }}=k_{p}=\frac{H^{2}}{12}$ is matched for $\Lambda=\frac{1}{8}$, as has been shown in Ref. [46].

Figure 32 displays $E(k)$ in $\mathrm{BF}$ for the two-layered periodic channel. All these results show a very similar dependency of $E(k)$ versus $\Lambda: E(k)$ noticeably reduces decreasing $\Lambda$ from $\frac{3}{8}$ to $\Lambda^{\text {min }}=10^{-2}$, from $\approx 30 \%$ to $\approx 3 \%$, on the coarse grid for $R_{k}=10^{3}$. Using $\Lambda^{\text {min }}$ only in the low-permeable layer also improves the results, from $\approx 30 \%$ to $\approx 8 \%$. This also applies for finer grids. On the other side, $E(k)$ reduces by a factor 6 from $H=12$ to 48 . When the permeability contrast $R_{k}$ increases from 10 to $10^{3}, E(k)$ increases much slower than linearly, by a factor 6 on the coarse grid and by a factor 4 on the fine. In $\mathrm{BF}$, the systematic permeability overestimate is confined to the Stokes-Brinkman domain because of the velocity raise into the open layer (cf. Fig. 10).

Figure 23 displays $E(k)$ for two asymmetric situations for $\mathrm{BF}$ and IBF together. First, the open layer is reduced to $h=2$ while the low-permeability porous layer is stretched to 46 . This linearly increases $E(k)$ from $20 \%$ to $60 \%$ for $\Lambda=\frac{3}{8}$ with respect to the case of equal layers of $h=H / 2=6$ in Fig. 32. The $\operatorname{BF}\left(\Lambda=\frac{1}{8}\right)$ has the same $E(k)$ as the $\operatorname{IBF}(\Lambda=$ $\left.\frac{3}{16}\right)$, outperformed by $\operatorname{IBF}\left(\Lambda=\frac{1}{8}\right)$. The BF reaches similar accuracy reducing $\Lambda$ to $10^{-2}$, but then the velocity field oscillates in low-permeable layer (second diagram). Second, the permeability ratio is inverted: $E(k)$ behaves similarly with $\sigma$ but its amplitude reduces by factor 50 for finely resolved fractured (open) layer.

We equate now symbolic solution $k^{\text {num }}\left(\Lambda, \sigma, R_{k}, R_{h}, H\right)$ to exact solution $k_{B}\left(\sigma, R_{k}, R_{h}\right)$ and derive the root $\Lambda\left(\sigma, R_{k}, R_{h}, H\right)$ with the help of a numerical routine. In $\mathrm{BF}$, this procedure works robustly only when $v_{i}$ is positive for all $\Lambda$, that is, when $\mathrm{Da}_{B}^{(i)}>\frac{1}{4 H^{2}}(\sigma \leqslant 2 H)$. This situation differs with the IBF where $\Lambda(\sigma)$ becomes available $\forall \sigma$. Figure 21 plots these solutions for BF and IBF in a single channel. As could be expected from the Poiseuille solution, $\left.\Lambda\right|_{\sigma \rightarrow 0} \rightarrow \frac{1}{8}$ in both schemes. Interestingly, $\Lambda$ reduces to zero with BF for $\sigma>\approx 8$ and, when $\sigma<2 H$, the distribution $\Lambda(\sigma)$ is practically the same for different $H$. In IBF, $\Lambda(\sigma, H)$ is not monotonous in the Brinkman zone; it first slightly increases inside the interval $\Lambda \in\left[\frac{1}{8}, \frac{3}{16}\right]$ and then decreases towards $\Lambda=0$. Unlike with $\mathrm{BF}, \Lambda(\sigma, H)$ increases with resolution. The increase of optimal $\Lambda$ with resolution has been observed in Ref. [45] for Stokes 

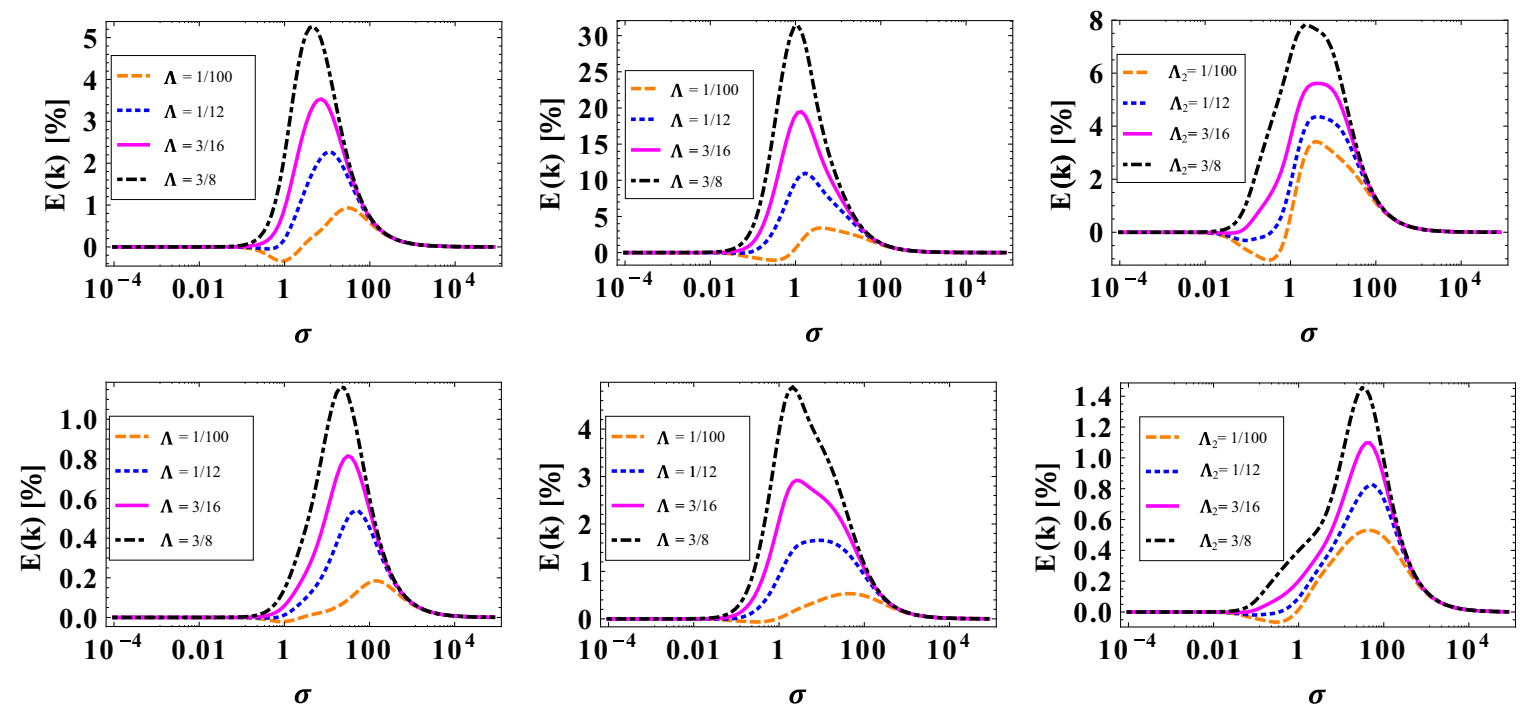

FIG. 32. (Color online) Predicted permeability error $E(k)$ in BF is plotted for the two-layered periodic channel with the contrast $r_{k}^{2}=$ $k_{1} / k_{2}=10^{-1}$ (left diagram) and $r_{k}^{2}=10^{-3}$ (middle and right diagrams) for two resolutions: $H=12$ (top row) and $H=48$ (bottom row). The two first columns apply $\Lambda_{1}=\Lambda_{2}=\left\{10^{-2}, \frac{1}{12}, \frac{3}{16}, \frac{3}{8}\right\} ; \Lambda_{1}$ is reduced to $10^{-2}$ for the low-permeable layer in the last column.

flow around regular and random arrays of spheres. In turn, the decrease towards $\Lambda=0$ is explained by the overestimation of the velocity on the flat Darcy profile. The right diagram in Fig. 21 confirms that $\Lambda=\frac{1}{8}$ is very accurate in the StokesBrinkman regime, followed by a nonmonotonous Brinkman zone where $\Lambda=\frac{1}{8}$ has to be increased towards $\Lambda=\frac{3}{16}, \Lambda \in$ $\left[\frac{1}{8}, \frac{3}{16}\right]$. Notice that IBF losses accuracy for very small $\Lambda$ in the Brinkman zone, in agreement with the velocity profiles in Fig. 15. In the Darcy regime, $E(k)$ is relatively small and similar for the three examined $\Lambda$; however, the exact solution reduces $\Lambda$ towards 0 .

Figure 22 depicts solutions $\Lambda\left(\sigma, R_{h}, H\right)$ in three two-layered situations explored above: a symmetric with equal width and the two antisymmetric ones with the aspect ratio $R_{h}=23$. The three situations show a similar dependency on $\Lambda\left(\sigma, R_{h}, H\right)$, but $\Lambda=\frac{1}{8}$ extends over a larger interval $\sigma$ when the fractured layer is not resolved (see in the right diagram). The IBF confirms the improvement of the BF profiles shown in Fig. 23.

Figure 24 compares $E(k)$ in $\mathrm{BF}, \mathrm{IBF}$, and FEM for single, bounded, and periodic channels of contrast $R_{k}=10$, resolved with the eight grid cells per layer. These results are extended to much larger contrasts, such as $10^{3}, 3 \times 10^{5}$, and $10^{9}$ for the periodic system in Fig. 25. The BF and IBF are applied with $\Lambda=\frac{1}{8}$. First, the inaccuracy of FEM in the Stokes-Brinkman regime $\sigma \in \approx] 0,1]$ in bounded channels is due to the "trapezoidal" integration rule while $\Lambda=\frac{1}{8}$ cures for this in LBM. In the Brinkman zone, $\left.\sigma \in \approx] 1,10^{2}\right],|E(k)|$ increases, and BF systematically overestimates permeability, indicating that $\Lambda$ should be reduced, in agreement with the predictions in Fig. 21. In that zone $E(k)$ is nonmonotonous for IBF, indicating that $\Lambda$ should first increase [when $E(k)<0$ ] and then decrease, in agreement with the results in Figs. 21 and 22. On the whole, the Darcy-Brinkman zone is predicted to be relatively accurate with IBF and BF for $\Lambda=\frac{1}{8}$. This is to be contrasted with FEM, which suffers from large oscillations there. Figure 25 shows that $|E(k)|$ increases together with $R_{k}$ for two LBM schemes and it covers a larger $\sigma$ subdomain.
This is consistent with the results in Fig. 10 which show very large velocity jumps on open-impermeable interface for very small $\sigma$. The positive side is that $|E(k)|$ increases by one order of magnitude when the permeability contrast increases by the eight orders. Typically, IBF with $\Lambda=\frac{1}{8}$ is 2 times more accurate than BF in the Stokes-Brinkman and Brinkman regimes for a very wide range of permeability contrasts. In FEM, $E(k)$ also increases for smaller $\sigma$ with $R_{k}$ but $E(k)$ remains negative and its amplitude is almost unaffected by permeability contrast. These results for $E(k)$ increase approximately linearly by reducing resolution to one grid cell per layer (in this particular case, BF and IBF coincide in periodic layers). Next we compare these two-layered results with the multilayered and two-dimensional predictions.

\section{B. Multilayered and two-dimensional random media}

Two- and three-dimensional log-normal distributions are commonly used, as advocated for their similarity with the porous media, e.g., Refs. [51,52]. Another advantage is their predictable Darcy permeability. Still, while the perturbation analysis around $k_{D}$ only applies for very smooth permeability fields [40], the apparent (Brinkman) permeability remains unpredicted. In general, it depends on the standard deviation and correlation length [40], in agreement with the estimates [1] for carbonates. In the present work, we limit ourselves to very weakly correlated two-dimensional Cauchy distribution depicted in Fig. 33 for its dispersive character, due to moments unboundness:

$$
f(k)=\frac{2 \gamma}{\pi\left(k^{2}+\gamma^{2}\right)}, \quad k>0, \quad \gamma>0, \quad k_{D}=e^{\langle\ln [k]\rangle}=\gamma .
$$

The effective Darcy permeability value $k_{D}$ for this distribution is predicted by Shwidler [53]. We examine four regimes sequentially reducing all permeability values by a factor of 100: $\sigma=1.52 \times 10^{n}, n=1, \ldots, 4$, in the $H^{2}=48 h^{2}$ box for two resolutions, $h=1$ and $h=8$. In these realizations, permeability varies by nine orders of magnitude, from 


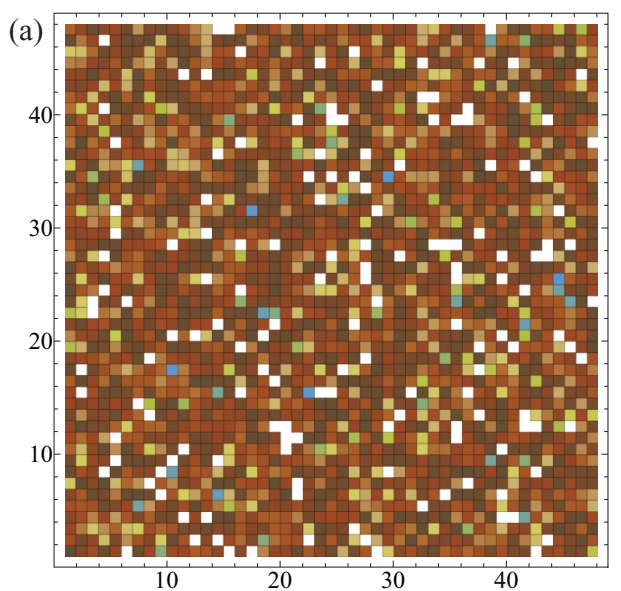

(b)

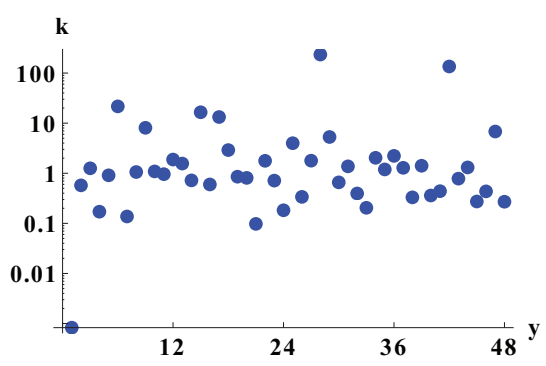

(c)

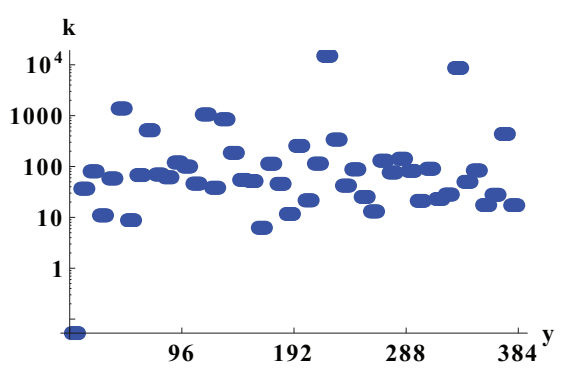

FIG. 33. (Color online) From the left to the right: (a) A two-dimensional Cauchy distribution in a $48^{2} h^{2}$ box with the largest permeability contrast over a sample $R_{k} \approx 10^{9}$, followed by one of its 48 multilayered subdistributions with (b) $h=1$ and (c) $h=8, R_{k} \approx 3 \times 10^{5}$.

$k^{\min }=3.4 \times 10^{-4} \times 10^{-2 n} h^{2} \quad$ to $\quad k^{\max }=3.6 \times 10^{5} \times$ $10^{-2 n} h^{2}, n=1, \ldots, 4$. The FEM finer-grid solution in the $(48 \times 16)^{2}$ box is employed as the reference one for $k_{B}$. The characteristic reference values $\sigma=\mathrm{Da}^{-1 / 2}$ and $R_{B}=\frac{k_{B}}{k_{P}}$ are reported in Table IV, along with the optimal intervals for $\Lambda(\sigma, h)$ where the IBF crosses the reference solution. The corresponding distribution $E(k)$ versus $\Lambda$ is plotted in Figs. 26-29 versus $\Lambda$ for $\mathrm{BF} / \mathrm{IBF}$ and compared to FEM on the same grid.

In parallel, we examine three multilayered samples: ML1, ML2, and ML3. In ML1, the permeability distribution is set equal to one column of the Cauchy distribution depicted in Fig. 33 and rescaled to obtain the same four values $k_{D}$ as have been prescribed in 2D via $\gamma$ (with $k_{D}$ equal to the arithmetical mean value in multilayers). The results in ML1 for $E(k)$ towards $\Lambda$ are displayed by the two last diagrams in Figs. 26-29. On the whole, the dependency $E(k)$ towards $\Lambda$ is very similar in 2D and ML1 and in qualitative agreement with the two-layered predictions:

(1) The dependency $E(k)$ versus $\Lambda$ is nonlinear in all four cases; the nonlinearity noticeably increases from (a) the Stokes-Brinkman to the Darcy regime, (b) the fine to coarse grid, (c) IBF to BF, and (d) multilayers to 2D, approaching the linear dependency proper to Poiseuille flow for ML1 on the fine grid in the Stokes-Brinkman regime $\sigma \approx 15.2$.

(2) The BF produces higher permeability values than the IBF for all $\Lambda \in] 0, \frac{3}{8}$ [ (recall that $\mathrm{BF}=\mathrm{IBF}$ for $\Lambda=\frac{3}{8}$ ) with larger $|E(k)|$ in interval $\Lambda \in \approx\left[\frac{1}{8}, \frac{3}{8}[\right.$. In ML1, the IBF with $\Lambda \in\left[\frac{1}{8}, \frac{3}{16}\right]$ matches the reference solution for $\sigma=15.2$ and $\sigma=152$ on the fine grid, followed by a slight $\Lambda$ decrease for $\sigma=1520$. In $2 \mathrm{D}$, the IBF slightly increases its optimal $\Lambda \in\left[\frac{1}{16}, \frac{1}{8}\right]$ between $\sigma=15.2$ and $\sigma=152$; in all IBF solutions, the optimal $\Lambda$ increases from a coarse to finer grid, in qualitative agreement with the two-layered predictions in Fig. 22.

(3) In the Darcy zone, the $\Lambda(\sigma)$ dependency becomes highly nonlinear with increasing of $\sigma$ and the optimal $\Lambda$ reduces sharply. In ML1, the IBF matches the exact solution for $\sigma=1.52 \times 10^{4}$ with $\Lambda \in\left[\frac{1}{32}, \frac{1}{16}\right]$ on the fine grid, while to achieve a similar result, the BF needs to reduce $\Lambda$ towards zero. In $2 \mathrm{D}, \Lambda$ reduces further, towards $\left[\frac{1}{512}, \frac{1}{64}\right]$ on the fine grid and $\left[10^{-6}, \frac{1}{512}\right]$ on the coarse; the $\mathrm{BF}$ does not reach the reference values even for $\Lambda \rightarrow 0$, in agreement with the predictions from their respective single-channel solutions in Fig. 21.

TABLE IV. This table provides approximate data for random 2D Cauchy distribution and three multilayered distributions, ML1, ML2, and ML3. In 2D: The reference solution $k_{B}$ is given by FEM on the finer grid $h=16$. In 1D: $k_{B}$ is the analytical multilayered solution.

\begin{tabular}{|c|c|c|c|c|c|c|c|c|}
\hline \multicolumn{5}{|c|}{ Random 2D Cauchy distribution } & \multicolumn{4}{|c|}{ ML3 } \\
\hline$\sigma=\mathrm{Da}^{-\frac{1}{2}}=\frac{48 h}{\sqrt{k_{0}}}$ & 15.2 & 152 & 1520 & 15200 & 856 & 2047 & 9129 & 21492 \\
\hline$R_{k}=k^{\max } / k^{\min D}$ & $10^{9}$ & $10^{9}$ & $10^{9}$ & $10^{9}$ & $10^{9}$ & $10^{9}$ & $10^{9}$ & $10^{9}$ \\
\hline$R_{B}=\frac{k_{B}}{k_{D}}$ & 0.3362 & 0.6796 & 0.925 & 0.97 & 0.3362 & 0.6796 & 0.925 & 0.97 \\
\hline Optimal $\Lambda(h=1)$ & $\frac{1}{16}$ & $\frac{1}{16}$ & {$\left[\frac{1}{256}, \frac{1}{128}\right]$} & {$\left[10^{-6}, \frac{1}{512}\right]$} & {$\left[\frac{3}{64}, \frac{1}{16}\right]$} & {$\left[\frac{1}{32}, \frac{3}{64}\right]$} & {$\left[\frac{1}{128}, \frac{1}{64}\right]$} & {$\left[\frac{1}{256}, \frac{1}{128}\right]$} \\
\hline Optimal $\Lambda(h=8)$ & $\frac{1}{16}$ & $\frac{1}{8}$ & {$\left[\frac{1}{32}, \frac{1}{16}\right]$} & {$\left[\frac{1}{512}, \frac{1}{64}\right]$} & $\frac{1}{32}$ & $\frac{1}{16}$ & {$\left[\frac{1}{32}, \frac{1}{16}\right]$} & {$\left[\frac{1}{32}, \frac{1}{16}\right]$} \\
\hline \multicolumn{5}{|c|}{ ML1 } & \multicolumn{4}{|c|}{ ML2 } \\
\hline$\sigma=\mathrm{Da}^{-\frac{1}{2}}=\frac{48 h}{\sqrt{k D}}$ & 15.2 & 152 & 1520 & 15200 & 281 & 915 & 4009 & 9786 \\
\hline$R_{k}=k^{\max } / k^{\min { }^{\sqrt{k} D}}$ & $3 \times 10^{5}$ & $3 \times 10^{5}$ & $3 \times 10^{5}$ & $3 \times 10^{5}$ & $3 \times 10^{5}$ & $3 \times 10^{5}$ & $3 \times 10^{5}$ & $3 \times 10^{5}$ \\
\hline$R_{B}=\frac{k_{B}}{k_{D}}$ & 0.09 & 0.23 & 0.8 & 0.98 & 0.3362 & 0.6796 & 0.925 & 0.97 \\
\hline Optimal $\Lambda(h=1)$ & $\frac{1}{8}$ & {$\left[\frac{1}{16}, \frac{1}{8}\right]$} & {$\left[\frac{1}{32}, \frac{1}{16}\right]$} & {$\left[\frac{1}{512}, \frac{1}{64}\right]$} & $\frac{5}{64}$ & {$\left[\frac{3}{64}, \frac{1}{16}\right]$} & {$\left[\frac{1}{64}, \frac{3}{128}\right]$} & {$\left[\frac{1}{128}, \frac{1}{64}\right]$} \\
\hline Optimal $\Lambda(h=8)$ & {$\left[\frac{1}{8}, \frac{3}{16}\right]$} & {$\left[\frac{1}{8}, \frac{3}{16}\right]$} & {$\left[\frac{1}{16}, \frac{1}{8}\right]$} & {$\left[\frac{1}{32}, \frac{1}{16}\right]$} & $\frac{1}{8}$ & {$\left[\frac{1}{16}, \frac{1}{8}\right]$} & $\frac{1}{8}$ & {$\left[\frac{1}{64}, \frac{3}{32}\right]$} \\
\hline
\end{tabular}


(4) In multilayers, FEM underestimates permeability in the Darcy zone by $60-70 \%$ for $\sigma=1.52 \times 10^{3}$ and $\sigma=$ $1.52 \times 10^{4}$, reducing $|E(k)|$ to $3-9 \%$, respectively, on the fine grid. This underestimate is related to the systematic lowering of the peak velocity values in the Darcy zone observable in Fig. 30 (fourth diagram in top row), together with the unphysical negative-valued oscillations in neighboring sites. In $2 \mathrm{D}$, the sign of $E(k)$ changes for $\sigma=1.52 \times 10^{4}$ where FEM overestimates $k_{B}$, similarly to the LBM, unless IBF is used with small $\Lambda$.

(5) In fact, the IBF operates with two space-dependent heterogeneous distributions, $\Lambda_{\star}^{+}(\sigma(\vec{r}), \Lambda)$ and $\Lambda_{\star}(\sigma(\vec{r}), \Lambda)$ (see in Table I); the corresponding distributions $\Lambda_{\star}(y)$ for fixed $\Lambda$ are depicted for four ML1 velocity profiles in the bottom row in Fig. 30. This figure shows that $\Lambda_{\star}(y)$ varies from its prescribed control value $\Lambda=\frac{1}{8}$ for $\sigma=15.2$ to $\Lambda_{\star}=\frac{3}{8}$ when $\sigma=15200$ [cf. Eqs. (55)]. At the same time, $\Lambda_{\star}^{+}$varies from $\Lambda^{+}$to $\frac{3}{8 \Lambda} \Lambda^{+}$, meaning an unbounded increase when $\Lambda \rightarrow 0$ in the Darcy zone [cf. Eqs. (56)]. The distribution $\Lambda_{\star}(y)$ is heterogeneous with $k(y)$ in two intermediate zones $\sigma=\{152,1520\}$.

On the whole, the permeability underestimate of about $5-10 \%$ by FEM agrees very reasonably with the two-layered predictions in the same resolution of eight nodes in Fig. 25 [recall that $E(k)$ in two layers is almost an independent permeability contrast with FEM]. This is to be contrasted with the $\operatorname{IBF}\left(\Lambda=\frac{1}{8}\right)$, where $E(k)$ does not exceed [-0.1,2]\% in multilayers for $h=8$ through the four regimes while its increase up to $10 \%$ has been predicted in Fig. 25 for a similar permeability ratio of order $3 \times 10^{5}$. This can be explained by the fact that the rapid increase in $E(k)$ is confined to a relatively small, flow-dependent interval of $\sigma$. In addition, when the sharp increase in $E(k)$ is caused by a large permeability contrast, the two-layered situation (side by side) is expected to be worse against the multilayered one.

Despite a visible similarity of the results in $2 \mathrm{D}$ and multilayers, the optimal $\Lambda$ in Table IV is systematically higher in ML1 than in 2D. At the same time, the obtained values $R_{B}=\frac{k_{B}}{k_{D}}$ are also higher in ML1, except in the Darcy zone, where $R_{B} \approx 1$. We hypothesize that the so-called flow factor [51], hereafter set equal to the effective value $R_{B}=\frac{k_{B}}{k_{D}}$, dictates the optimal $\Lambda$ and not the averaged Darcy value described by $\sigma$. We then consider two other distributions, ML2 and ML3. In four realizations of ML2, ML1 is rescaled to match four reference values $R_{B}$, previously obtained in four realizations of two-dimensional random samples. In ML3, $k^{\text {min }}$ and $k^{\text {max }}$ are additionally updated to assure the same largest contrast $R_{k} \approx 10^{9}$ as in $2 \mathrm{D}$. The analytical dependency $k_{B}\left(k_{D}\right)$ is nonlinear in multilayers and the four scale factors are derived with the help of a numerical routine. In turn, $k_{D}$ and hence $\mathrm{Da}=k_{D} / H^{2}$ now differ in multilayers and 2D. This analysis focuses on the IBF scheme.

The four coarse-grid distributions $E(k)$ versus $\Lambda$ are plotted together for ML2, ML3, and 2D in Fig. 31. Their characteristic values and effective solutions for $\Lambda(h)$ are summarized in Table IV. In the Stokes-Brinkman $\left(R_{B} \approx 0.34\right)$ and Brinkman $\left(R_{B} \approx 0.68\right)$ regimes, the optimal solution $\Lambda(2 \mathrm{D})$ is found between ML2 and ML3. In the Darcy-Brinkman regime $\left(R_{B} \approx 0.925\right)$, the ML3 covers the optimal value the best. In the Darcy zone $\left(R_{B} \approx 0.97\right)$, when $\Lambda(2 \mathrm{D}) \approx 0$, the ML3 overestimates it.
In summary, this last numerical experiment confirms that it is reasonable to build a strategy for "upscaling" the multilayered, optimal $\Lambda$ in similar stochastic distributions based on $k_{B} / k_{D}$ ratios in two systems. In practice, this suggests an iterative adjustment for $R_{B}(\Lambda)$ between oneand multidimensional samples. This assumes that $k_{D}$ values have been predicted or precomputed for both systems. We emphasize that all results in random media have been obtained without solid inclusions, meaning that all the observed dependencies on the free parameter $\Lambda$ originate from bulk and interfaces.

Concerning the BF, IBF, and FEM, their solutions are quite similar in the intermediate Stokes-Brinkman zones for relatively fine resolution, demonstrated by three multilayered profiles in Fig. 30. However, they may become quite different on the open-impermeable interface, where LBM undergoes $\Lambda$-dependent slip-velocity jumps, and in the Darcy limit on coarse grids. The velocity profiles in the Darcy regime illustrated for this in Figs. 18 and 20 and in the last diagram in Fig. 30. They show that the most noticeable numerical artifacts of the FEM solutions are observable in the Darcy-Brinkman regime where oscillating accommodation of the profiles results in lowering of the pick velocities and neighbor-node negative velocities in multilayers, giving rise to a significant permeability underestimate shown in Figs. 24, 25, 28, and 29 (last two diagrams).

Improvements with the space resolution have been reached, however, for all regimes. We refer to Ref. [22] for improving of the unresolved boundary layers in the Darcy limit with the stabilizing elements and/or adaptive grid refining. Eventually, based on such strategies, FEM might outperform LBM. Still, we stress that, besides the overall simplicity, (i) the implicit boundary layers help the LBM, (ii) the LBM controls the convergence to steady state more easily (see in Sec. VIC), and (iii) its extension to the adaptive grid is also possible. In this last point we mention nonuniform grid extension [31] and a recent finite-volume LBM formulation [30] for Brinkman equation.

\section{The convergence time}

In LBM, the time convergence to steady state is differently governed by the selected viscosity value in Stokes flow for regular and random media [44-46]. We have found that the following semiheuristic rule is valid in LBM: The same order of the time steps is required for very different regimes, and on the fine and coarse grids, provided that the product $\sigma_{\max } v$ or $\sigma_{\max }^{2} v$ remains approximately the same. In fact, this rule aims to approximately preserve the largest amplitude of the resistance factor $B_{f}=v / k^{\mathrm{min}}$ (in lattice units) through the series of computations. In practice, $\Lambda^{+}$should decrease accordingly from the Stokes to the Darcy regime but reduction below $10^{-5}-10^{-6}$ becomes inefficient. Applied LBM stopping criteria was permeability based, as $\left|k\left(t+\delta_{t}\right) / k(t)-1\right|<\varepsilon$, with $\varepsilon \approx 10^{-12}$ and $\delta_{t} \approx 10^{4}$. Generally, IBF converged faster than $\mathrm{BF}$ and, for small $v$, very small $\Lambda$ converged faster.

The BGK operator takes the same computational time as the TRT, but the obtained permeability changes with $\nu$, lacking physical consistency. Obviously, if BF-BGK employs $v=\sqrt{\Lambda} / 3$, the same results as with $\operatorname{BF}-\mathrm{TRT}(\Lambda)$ are obtained. 
However, the TRT endorses them for any $v$ value when $\Lambda$ is fixed. Therefore, even if we may recognize that the BGK supports the general trend of these simulations (small $\Lambda$ and small $v$ in the Darcy regime, with both $\Lambda$ and $v$ increasing towards the Stokes regime), the BGK cannot offer the control of accuracy independently of the convergence time. Besides, it is clear that the BGK operator is unable to offer a functionality of IBF where the two effective relaxation rates should differ.

We have also recognized a noticeable increase in the number of time steps from the coarse to fine grid in random media with the COMSOL FEM software using the GMRES iterative solver (without preconditioner). Here the stopping criteria are based on the error estimate, which checks whether the relative residual (times some stability constant) is less than a prescribed tolerance (set to be $10^{-10}$ in this work).

In summary, we notice that neither one of the two used criteria guarantees the steady state in the sense that the fluid mass flux is constant over the media (see Refs. [44-46]). In LBM, this last criteria is natural but it takes much more time to converge without any distinguishable difference for permeability in present simulations. Our preliminary simulations suggest an advantage of IBF-TRT over FEM on relatively fine grids, given that the LBM is rather simple to make efficient and it allows for accuracy improvement with the free-tunable relaxation rate, due to implicit interface and boundary layers.

\section{CONCLUDING REMARKS}

The present work aimed to show up to what extent the "small" details of the LBM population update can affect the results in porous flow. Particular focus has been devoted to the following three points: (i) whether the population update and boundary conditions respect the nondimensional groups of the modeled equation, (ii) if the macroscopic numerical solutions satisfy the limit configurations, and (iii) the role of free numerical parameters.

Concerning point (i), we have shown that the BF and new IBF models respect the nondimensional groups using the bounce-back rule, when $\Lambda$ distribution is fixed on the given grid. This is in contrast with the local gray schemes [35-38] which make the modeled equations nonlinear with respect the kinematic viscosity. Future work needs to verify nonlocal link-based modeling of the resistance with respect to this issue [32-34].

Concerning point (ii), we stress that neither the ChapmanEnskog nor the asymptotic analysis has been used in this work: The results in series and parallel channels are based on the exact symbolic solutions constructed for all examined schemes. We have demonstrated that all LBM schemes need to redefine the microscopic momentum with the half forcing to match the constant velocity in the Darcy limit. A subtle point that we have to deal with was to connect the bulk recurrence equations [43] of the TRT operator at the interface. We show that they remain valid on the virtual linkwise continuation of the population components over it. The constructed solutions extend the known class of the analytical steady-state LBM solutions in the presence of the boundary and interfaces. They allow us to visualize velocity slip on the nondiscretized interface or boundary and to predict permeability in continuous space of the governing parameters, such as the Darcy number, aspect ratios, or permeability contrasts. The extension of our methodology for other-type channel flow in the presence of the variable collision or forcing components, such as the Brinkman-Forchheimer flow, two-phase-flow, or immersed boundary method, is possible. Furthermore, this solution technique has been recently extended for transient, advectiondiffusion solutions in heterogeneous stratified soil [54].

Despite the simplicity of channel flow, it reveals a number of critical properties of the two analyzed numerical approaches. One of them is the complex interplay between the bulk and interface or boundary effective discretizations. In all schemes, the discontinuous prefactor $1+\delta$ of viscosity correction multiplies, for two interface sides, the shear stress condition and, additionally, the two coefficients in Taylor-type velocity conditions for the BF scheme. Basically, the interface displays two different scenarios in the Stokes-Brinkman and DarcyBrinkman regimes. The FEM builds continuous solutions, only slightly deficient in the Stokes-Brinkman domain because of the relatively insignificant negative numerical viscosity. In the Darcy-Brinkman regime, its amplitude increases and the FEM amplifies velocity fluctuations due to an attempt of the exact coupling of the discontinuous Darcy branches for unresolved boundary layers. This is opposed to LBM where the Darcy branches coexist almost "independently," making their coupling and accommodation to the solid boundary managed by the "invisible" interface or boundary layers.

Concerning point (iii), it is focused on the highly nonlinear dependency $k(\Lambda)$. It shows that the optimal choice for the controlling combination $\Lambda$ becomes especially crucial because of the heterogeneity. The difficulty is that the optimal $\Lambda$ depends on the flow regime, ranging from the Poiseuille choice in the Stokes-Brinkman regime, around $\Lambda \in\left[\frac{1}{8}, \frac{3}{16}\right]$ for permeability, to zero in the Darcy flow where, unlike in the $\mathrm{BF}$ and FEM, the IBF may then reach reference values on the very coarse grid. This is due to the fact that the prescribed distribution of the symmetric-mode viscosity function $\Lambda_{\star}^{+}$automatically varies in heterogeneous media with $B=f(\phi) / k$. Another new point is that $\Lambda_{\star}^{+}$depends on the free antisymmetric-modes eigenfunction $\Lambda^{-}$, thereby, unusually, now $\Lambda_{\star}=\Lambda_{\star}^{+} \Lambda^{-}$nonlinearly depends on $\Lambda^{-}$. For example, when $\Lambda^{-} \rightarrow 0$, then $\Lambda_{\star} \rightarrow \frac{3}{8}$ in the Darcy limit $B \rightarrow \infty$, where $\Lambda_{\star}^{+}$increases infinitely. It is clear that the BGK operator is unable to offer a functionality of the IBF-TRT where the two relaxation functions, $\Lambda_{\star}^{+}$and $\Lambda^{-}$, may tend to the opposite limits for the sake of accuracy. Besides, if the convergence to steady state drastically depends on the selected viscosity value $v$ in Stokes computations $[45,46]$ where the optimal viscosity choice is structure dependent, it depends on the Darcy numbers in the Brinkman model. Using the simple practical argument, the same permeability value for any viscosity $v$ is vital when searching for optimal performance. This is unavailable with the BGK operator. In MRT collisions [55], the IBF determines the relationship between the kinematic viscosity eigenfunction $\Lambda_{\star}^{+}(v)$ and the common eigenfunction $\Lambda^{-}$of the antisymmetric modes. Additional free-tunable eigenfunctions of symmetric modes have to vary in fixed proportion with $\Lambda_{\star}^{+}$ when $v$ is altered [45].

The two-dimensional flow around the permeable cylinders, examined in Ref. [56], confirms that the IBF effectively suppresses strong interface fluctuations typical in BF and 
FEM. The random and bimodal two-dimensional benchmarks are of the special interest in this context, since they provide nontrivial validation to scheme (54), which has been derived for the straight channel. The heuristic rule for the choice of the reliable $\Lambda$ consists in iterative equating of the flow factors $k_{B} / k_{D}$ for "simple" (eventually multilayered) and "complex" porous rock for similar stochastic properties in permeability distributions. The usefulness of this rationale is that in multilayers exact solutions are known. On the other side, the flow factor is related to the stochastic characteristics of the media and some specific integration of the velocity field via second-order perturbation analysis around $k_{D}$ [40]. We can think about future extensions of these results to validate our numerical strategy in CT scans and anisotropic permeability distributions.

Finally, one may depreciate the above efforts to improve the low-permeable Darcy zones by arguing that they have a negligible contribution to the effective permeability itself, e.g., Ref. [31]. However, we believe that the accurate modeling of the interfaces between open and micritic phase is important, since the solute may be transported there and reside for a long time. Although being neglected by Stokes flow modeling in Portland carbonates [57,58], low-porosity zones are expected to play a role in producing sharp peaks and long tails in breakthrough curves because of the large porosity or permeability contrast [54,59]. In view of these phenomena, the simple strategy proposed here, aimed at damping the interface velocity fluctuations via the IBF, might turn out to be indispensable. Complementarily, when the solid boundary or interface is well resolved or has a prescribed curved shape, one might think about further improving the Brinkman schemes via explicit high-order boundary and interface conditions. In our opinion, the multireflection [44] or equivalent boundary approaches $[45,48]$ have to be "upscaled" to capture for the new $\Lambda$ effect in Brinkman modeling, which is already present in the first-order Taylor boundary expansion because of resistance variation. The main advantage of such conditions would be in making flow dependency on $\Lambda$ negligible. A similar scenario is interesting for the interface, perhaps extending the ideas of explicit conditions developed for discontinuous collision components in two-phase models $[47,60]$ and advection-diffusion equations with heterogeneous coefficients [47,61-63]. The subsequent research path might be focused on the impact of the obtained Brinkman flow-field quality on the dispersion.

\section{ACKNOWLEDGMENTS}

The authors are grateful to ANR for funding the project LaboCothep ANR-12-MONU0011 and to Dr. D. Bauer for useful discussions.

\section{APPENDIX A: DISCRETIZED MULTILAYERS}

The LBM operates on the cell-centered grid $y_{j}=Y_{i-1}+$ $\frac{1}{2}+j, j=0,1,2, \ldots, h_{i}-1$ inside each layer $y \in\left[Y_{i-1}, Y_{i}\right]$; the interface or boundary is not discretized. The FEM is discretized on the element vertexes $y_{j}=Y_{i-1}+j, j=$ $0,1,2, \ldots, h_{i}$; the interface or boundary is discretized. The space step is set equal to 1 . The discretized bulk solution given by Eqs. (34) satisfies the two following relations:

$$
\bar{\Delta}_{y} r^{ \pm y}= \pm \frac{\left(r^{2}-1\right)}{2} r^{(-1 \pm y)}, \quad \bar{\Delta}_{y}^{2} r^{ \pm y}=(r-1)^{2} r^{(-1 \pm y)}
$$

\section{TRT schemes}

In a single channel, the closure relation (35) becomes, with help of Eqs. (A1) in two boundary nodes $y_{b}= \pm \frac{H}{2} \mp \frac{1}{2}$, respectively (we drop index $i$ and replace $a_{i}, c_{i}$ by $a^{\text {num }}, c^{\text {num}}$ ),

$$
\begin{array}{r}
a_{11} a^{\text {num }}+a_{12} c^{\text {num }}=1, \quad a_{12} a^{\text {num }}+a_{11} c^{\text {num }}=1, \\
a_{11}=\frac{r^{\frac{-3+H}{2}}}{8}\left[8 r+2 \alpha^{+}\left(r^{2}-1\right)+\alpha^{-}(r-1)^{2}\right], \\
a_{12}=\frac{r^{-\frac{1+H}{2}}}{8}\left[8 r-2 \alpha^{+}\left(r^{2}-1\right)+\alpha^{-}(r-1)^{2}\right] .
\end{array}
$$

By symmetry, $a^{\text {num }}=c^{\text {num }}$ and $U_{j}$ reads

$$
\begin{aligned}
\text { TRT }: U_{j} & =a^{\text {num }} r^{-y_{j}}\left(1+r^{2 y_{j}}\right), \quad y_{j}=-\frac{H}{2}+\frac{1}{2}+j, \ldots, \frac{H}{2}-\frac{1}{2}, \quad j=0,1, \ldots \\
a^{\text {num }} & =\frac{8 r^{(3+H) / 2}}{2 \alpha^{+}\left(r^{2}-1\right)\left(r^{H}-r\right)+\left(\alpha^{-}(r-1)^{2}+8 r\right)\left(r+r^{H}\right)} .
\end{aligned}
$$

In multilayers, two coefficients per layer, $a_{i}$ and $c_{i}$, are to be derived from the linear system corresponding to interface velocity and stress conditions, respectively:

$$
\begin{aligned}
& a_{11}^{(i)} a_{i}+a_{12}^{(i)} c_{i}-\left(a_{11}^{(i+1)} a_{i+1}+a_{12}^{(i+1)} c_{i+1}\right)=\frac{\mathrm{Da}_{i}-\mathrm{Da}_{i+1}}{\mathrm{Da}} \\
& a_{21}^{(i)} a_{i}+a_{22}^{(i)} c_{i}-\left(a_{21}^{(i+1)} a_{i+1}+a_{22}^{(i+1)} c_{i+1}\right)=0 .
\end{aligned}
$$

The two interface conditions are given by Eqs. (36b) and (36a), respectively, where, applying finite-difference operators (A1) to velocity form (34), the four coefficients read in
Eq. (A4):

$$
\begin{aligned}
& a_{11}^{(i)}=\frac{r_{i}^{Y_{i}-\frac{3}{2}}}{8}\left[8 r_{i}+2 \alpha_{i}^{+}\left(r_{i}^{2}-1\right)+\alpha_{i}^{-}\left(r_{i}-1\right)^{2}\right], \\
& a_{12}^{(i)}=\frac{r_{i}^{-Y_{i}-\frac{1}{2}}}{8}\left[8 r_{i}-2 \alpha_{i}^{+}\left(r_{i}^{2}-1\right)+\alpha_{i}^{-}\left(r_{i}-1\right)^{2}\right], \\
& a_{21}^{(i)}=-\frac{\left(1+\delta_{i}\right)\left(r_{i}-1\right) r_{i}^{Y_{i}-\frac{1}{2}}}{f_{i}(\phi)}, \\
& a_{22}^{(i)}=\frac{\left(1+\delta_{i}\right)\left(r_{i}-1\right) r_{i}^{-Y_{i}-\frac{1}{2}}}{f_{i}(\phi)} .
\end{aligned}
$$


In bounded system, the bounce-back closure relation (A2) completes the system for $r=r_{N}$ and $r=r_{1}$. In the periodic system, interface conditions relate the first and last layers.
In two-layered system, alternatively, the symmetry conditions can be applied at the middle of each layer. Assuming interface at $Y=0$, solution of Eqs. (A4) reads

$$
\begin{aligned}
a_{1} & =\frac{8\left(\mathrm{Da}_{1}-\mathrm{Da}_{2}\right)}{\mathrm{Da}} f_{1}(\phi) r_{1}^{\frac{3+2 h_{1}}{2}} \frac{p_{1}\left(r_{2}, h_{2}, \delta_{2}\right)}{p_{3}}, \quad c_{1}=r_{1}^{-h_{1}} a_{1}, \\
c_{2} & =\frac{8\left(\mathrm{Da}_{2}-\mathrm{Da}_{1}\right)}{\mathrm{Da}} f_{2}(\phi) r_{2}^{\frac{3+2 h_{2}}{2}} \frac{p_{1}\left(r_{1}, h_{1}, \delta_{1}\right)}{p_{3}}, \quad a_{2}=r_{2}^{-h_{2}} c_{2}, \\
p_{3} & =f_{1}(\phi) p_{1}\left(r_{2}, h_{2}, \delta_{2}\right) p_{2}\left(r_{1}, h_{1}, \delta_{1}\right)+f_{2}(\phi) p_{1}\left(r_{1}, h_{1}, \delta_{1}\right) p_{2}\left(r_{2}, h_{2}, \delta_{2}\right), \\
p_{2} & =2 \alpha^{+}\left(r^{2}-1\right)\left(r^{h}-r\right)+\left[\alpha^{-}(-1+r)^{2}+8 r\right]\left(r+r^{h}\right), \\
p_{1}(r, h, \delta) & =(r-1) r\left(r^{h}-1\right)(1+\delta), \quad X=1+\delta .
\end{aligned}
$$

This solution is valid applying Eq. (34b) with the individual solutions for $\delta_{i}$ and $\alpha_{i}^{ \pm}$. Velocity values are given by Eq. (34), and their summation yields in Eq. (24):

$$
\begin{aligned}
k_{r}^{\mathrm{num}}= & -\frac{r_{1}^{\left(\frac{1}{2}-h_{1}\right)}\left(r_{1}^{h_{1}}-1\right)\left(a_{1}+c_{1} r_{1}^{h_{1}}\right)}{H\left(r_{1}-1\right)} \\
& -\frac{r_{2}^{\left(\frac{1}{2}-h_{2}\right)}\left(r_{2}^{h_{2}}-1\right)\left(c_{2}+a_{2} r_{2}^{h_{2}}\right)}{H\left(r_{2}-1\right)} .
\end{aligned}
$$

Remark. The BF, IBF, and IFD solutions differ in general. One exception is the one-node-per-layer system where BF and IBF coincide and then reduce to IFD for $\Lambda_{i}=\frac{3}{16}$. The onenode-layer solution in the two-layered system (with $k_{3}=k_{1}$ ) reads

$$
\begin{aligned}
\mathrm{BF} / \mathrm{IBF}: U_{j}^{(i)} & =\frac{1}{4 \mathrm{Da}} \frac{3 k_{i}\left(k_{i+1}-k_{i}\right)}{3\left(k_{1}+k_{2}\right)+2\left[\Lambda_{1} f_{1}(\phi)+\Lambda_{2} f_{2}(\phi)\right]} . \\
\mathrm{IFD}: U_{j}^{(i)} & =\frac{1}{4 \mathrm{Da}} \frac{2 k_{i}\left(k_{i+1}-k_{i}\right)}{8\left(k_{1}+k_{2}\right)+f_{1}(\phi)+f_{2}(\phi)}, \\
i & =1,2, \quad j=1,2 .
\end{aligned}
$$

The three solutions also coincide for $\Lambda=\frac{3}{16}$ in the single channels $H=1$ or $H=2$ and then in the two-layered bounded channel with $H=2$ for BF and IFD.

\section{The FEM scheme}

In the single channel, solution of the FEM is given by Eq. (34) for $\delta=-B / 6$. The coefficient $a^{\text {num }}=c^{\text {num }}$ is set by equating $u_{j}$ to zero at $y_{j}= \pm \frac{H}{2}$ :

$$
\begin{aligned}
\text { FEM : } U_{j} & =-\frac{r^{\frac{H}{2}-y_{j}}+r^{\frac{H}{2}+y_{j}}}{1+r^{H}}, \\
y_{j} & =-\frac{H}{2},-\frac{H}{2}+1, \ldots, \frac{H}{2} .
\end{aligned}
$$

In multilayers, the coefficients in Eqs. (A4) are set from the velocity and stress continuity condition (69) applied on solution (34):

$$
\begin{aligned}
& a_{11}^{(i)}=r_{i}^{Y_{i}}, \quad a_{12}^{(i)}=r_{i}^{-Y_{i}}, \quad a_{21}^{(i)}=-\frac{\left(1+\delta_{i}\right)\left(r_{i}^{2}-1\right) r_{i}^{Y_{i}-1}}{2 f_{i}(\phi)}, \\
& a_{22}^{(i)}=\frac{\left(1+\delta_{i}\right)\left(r_{i}^{2}-1\right) r_{i}^{-Y_{i}-1}}{2 f_{i}(\phi)} .
\end{aligned}
$$

\section{APPENDIX B: THE RECURRENCE EQUATIONS FOR MODIFIED SCHEMES}

We consider the modified schemes (50) in the equivalent form:

$$
\begin{aligned}
\tilde{f}_{q}(\vec{r}, t) & =f_{q}(\vec{r}, t)+\mathcal{G}_{q}^{+}+\mathcal{G}_{q}^{-}, \\
\mathcal{G}_{q}^{ \pm} & =-s^{ \pm}\left(f_{q}^{ \pm}-\mathcal{E}_{q}^{ \pm}\right), \text {where } \\
\mathcal{E}_{q}^{+} & =e_{q}^{+}+\frac{A^{+}}{s^{+}} g_{q}^{+}, \quad \mathcal{E}_{q}^{-}=e_{q}^{-}+\frac{A^{-}\left(g_{q}^{-}-F_{q}\right)}{s^{-}} .
\end{aligned}
$$

Here the equilibrium function $e_{q}^{ \pm}$, the postcollision quantities $g_{q}^{ \pm}=-s^{ \pm}\left(f_{q}^{ \pm}-e_{q}^{ \pm}\right)$, and the external forcing $F_{q}$ are defined as in Eqs. (7) and (37). The recurrence equations (5) and (6) remain valid, replacing $e_{q}^{ \pm}$with $\mathcal{E}_{q}^{ \pm}$and $g_{q}^{ \pm}$with $\mathcal{G}_{q}^{ \pm}$. Mass and momentum conservation equations (10) become $\sum_{q=0}^{Q-1} \mathcal{G}_{q}^{+}=0$ and $\sum_{q=1}^{Q_{m}} \mathcal{G}_{q}^{-} \vec{c}_{q}=\vec{F}$. One new term $T_{1}$ in the right-hand side of the momentum equation is due to correction $\frac{A^{+}}{s^{+}} g_{q}^{+}$in $\bar{\Delta}_{q} \mathcal{E}_{q}^{+}$[see Eq. (5b)]: $T_{1}=-2 \sum_{q=1}^{Q_{m} / 2} \frac{A^{+}}{s^{+}} \bar{\Delta}_{q} g_{q}^{+} \vec{c}_{q}$. Using $\mathcal{G}_{q}^{+}=g_{q}^{+}+A^{+} g_{q}^{+}, g_{q}^{+}=\mathcal{G}_{q}^{+} /\left(1+A^{+}\right)$, and $\bar{\Delta}_{q} g_{q}^{+}=$ $\bar{\Delta}_{q} \mathcal{G}_{q}^{+} /\left(1+A^{+}\right)$, where $\bar{\Delta}_{q} \mathcal{G}_{q}^{+}$is expressed with Eq. (6a), we obtain

$$
\begin{aligned}
& T_{1}=-2 a^{+} \sum_{q=1}^{Q_{m} / 2}\left(\bar{\Delta}_{q}^{2} \mathcal{E}_{q}^{-}-\Lambda^{-} \bar{\Delta}_{q}^{2} \mathcal{G}_{q}^{-}\right) \vec{c}_{q}, \\
& a^{+}=\frac{A^{+}}{\left(1+A^{+}\right) s^{+}}=\frac{A^{+}\left(1+2 \Lambda^{+}\right)}{2\left(1+A^{+}\right)} .
\end{aligned}
$$

Substituting $\mathcal{E}_{q}^{-}$from Eq. (B1) and $F_{q}$ from Eq. (7), this term can be computed similarly as in Eq. (38):

$$
\begin{aligned}
T_{1}= & -a^{+}\left\{\frac{1}{3}\left(1-\Lambda^{-} B_{f}\right) \bar{\Delta}^{2} \vec{\jmath}\right. \\
& \left.+2 \Lambda^{-} \sum_{q=1}^{Q_{m} / 2} \bar{\Delta}_{q}^{2}\left[\frac{A^{-}}{s^{-}}\left(g_{q}^{-}-F_{q}\right)-\mathcal{G}_{q}^{-}\right]\right\} \vec{c}_{q},
\end{aligned}
$$

with

$$
g_{q}^{-}=\left(\mathcal{G}_{q}^{-}+A^{-} F_{q}\right) /\left(1+A^{-}\right) .
$$


The second new term $T_{2}$ appears in the right-hand side from $\Lambda^{+} \bar{\Delta}_{q}^{2} \mathcal{E}_{q}^{-}$:

$$
T_{2}=2 \Lambda^{+} \frac{A^{-}}{s^{-}} \sum_{q=1}^{Q_{m} / 2} \bar{\Delta}_{q}^{2}\left(g_{q}^{-}-F_{q}\right) \vec{c}_{q} .
$$

Next, in order to construct an exact solution for these schemes, one needs to prescribe $\mathcal{G}_{q}^{-}$.

\section{Stratified channel}

In a stratified channel, $\mathcal{G}_{q}^{-}(y)$ is given by Eq. (26) because of the streamwise conditions and momentum conservation. Then Eq. (B4) allows us to express $g_{q}^{-}(y)$. When $A^{-}=0, T_{2}$ vanishes and $T_{1}$ becomes in Eq. (B3):

$$
\begin{aligned}
A^{-}=0: T_{1} & =\frac{\Lambda^{+}}{3} \delta^{\star}\left(A^{+}\right) \bar{\Delta}_{y}^{2} j_{x}(y), \\
\delta^{\star}\left(A^{+}\right) & =\frac{-A^{+}\left(1+2 \Lambda^{+}\right)(3+2 B \Lambda)}{6\left(1+A^{+}\right) \Lambda^{+}} .
\end{aligned}
$$

The effective viscosity coefficient becomes equal to $\frac{\Lambda^{+}}{3}[1+$ $\left.\delta_{\star}\left(A^{+}\right)\right]$with

$$
A^{-}=0: \delta_{\star}\left(A^{ \pm}\right)=\delta+\delta^{\star}\left(A^{+}\right) .
$$

Here $\delta$ is given by Eq. (33) and $\delta^{\star}\left(A^{+}\right)$is given by Eq. (B6). When $A^{-} \neq 0$, substituting $g_{q}^{-}$from Eq. (B4) alone with Eq. (26) for $\mathcal{G}_{q}^{-}(y)$ into Eqs. (B3) and (B5), we obtain:

$$
\begin{aligned}
T_{1} & =\frac{\Lambda^{+}}{3} \delta^{\star}\left(A^{ \pm}\right) \bar{\Delta}_{y}^{2} j_{x}(y), \\
T_{2} & =\frac{\Lambda^{+}}{3} \delta^{\star}\left(A^{-}\right) \bar{\Delta}_{y}^{2} j_{x}(y), \\
\delta^{\star}\left(A^{-}\right) & =-\frac{A^{-} B\left(\Lambda^{+}+2 \Lambda\right)}{3\left(1+A^{-}\right)}, \\
\delta^{\star}\left(A^{ \pm}\right) & =\frac{A^{+}\left(1+2 \Lambda^{+}\right)\left(-3+A^{-}\left(-3+B \Lambda^{+}\right)-2 B \Lambda\right)}{6\left(1+A^{-}\right)\left(1+A^{+}\right) \Lambda^{+}} .
\end{aligned}
$$

The effective viscosity of the modified scheme (B1) is $\frac{\Lambda^{+}}{3}\left[1+\delta_{\star}\left(A^{ \pm}\right)\right]$, where the relative viscosity correction $\delta_{\star}\left(A^{ \pm}\right)$now sums three contributions given by Eq. (31b) and Eq. (B8):

$$
\delta_{\star}\left(A^{ \pm}\right)=\delta+\delta^{\star}\left(A^{ \pm}\right)+\delta^{\star}\left(A^{-}\right) .
$$

In principle, this allows for two free coefficients $A^{+}$and $A^{-}$ equating $\delta_{\star}\left(A^{ \pm}\right)$to zero. In particular, one obtains the $\mathrm{BF}^{-}$ scheme (51) when $A^{+}=0$ and the $\mathrm{BF}^{+}$scheme (53) when $A^{-}=0$.

\section{Interface or boundary conditions}

We replace $e_{q}^{ \pm}$and $g_{q}^{ \pm}$with $\mathcal{E}_{q}^{ \pm}$and $\mathcal{G}_{q}^{ \pm}$, respectively, in interface conditions (13). The solution for $\mathcal{G}_{q}^{-}$has been discussed above. Keeping in mind that in channel flow $\bar{\Delta}_{q}^{2} e_{q}^{+}=0$, then, from Eq. (6a), $\bar{\Delta}_{q}^{2} \mathcal{G}_{q}^{+}=\frac{\bar{\Delta}_{q} \mathcal{G}_{q}^{-}}{a^{+}-\Lambda^{+}}$, where $a^{+}$ is given by Eq. (B2), and $\mathcal{G}_{q}^{+}$has the following solution from Eq. (5a):

$$
\mathcal{G}_{q}^{+}=\bar{\Delta}_{q} \mathcal{E}_{q}^{-}+\left[-a^{+} \Lambda^{-}+\left(\Lambda-\frac{1}{4}\right)\right] \bar{\Delta}_{q}^{2} \mathcal{G}_{q}^{+} .
$$

The velocity condition (36b) reads with

$$
\begin{aligned}
& \alpha^{+}=-\frac{2\left(1+A^{+}\right) \Lambda^{+}}{A^{+}-2 \Lambda^{+}}\left(1+\delta_{\star}\right), \\
& \alpha^{-}=-\frac{8\left(A^{-} \Lambda^{+}-2 \Lambda\right)}{3\left(1+A^{-}\right)}\left(1+\delta_{\star}\right),
\end{aligned}
$$

where $\delta_{\star}=\delta_{\star}\left(A^{ \pm}\right)$is given by Eq. (B9). The stress-continuity condition (36a) is described with $v_{i}=\frac{\Lambda^{+}}{3}\left[1+\delta_{\star}\left(A_{i}^{ \pm}\right)\right]$.

\section{Improved schemes with $\delta_{\star}\left(A^{ \pm}\right)=0$}

We require $\delta_{\star}\left(A^{ \pm}\right)=0$, and this relates $A^{+}$to $A^{-}$in a specific way:

$$
\delta_{\star}\left(A^{ \pm}\right)=0: A^{+}\left(A^{-}\right)=-\frac{B \Lambda^{+}\left[3+A^{-}\left(3+4 \Lambda^{+}\right)-8 \Lambda\right]}{\left\{6+3(4+B) \Lambda^{+}+A^{-}\left[6+(12+B) \Lambda^{+}\right]+4 B \Lambda\right\}},
$$

where the coefficients in the velocity closure relation are given by the following relations:

$$
\alpha^{+}=\frac{-4\left[-3+A^{-}\left(-3+B \Lambda^{+}\right)-2 B \Lambda\right]}{3\left(1+A^{-}\right)(4+B)}, \quad \alpha^{-}=-\frac{8\left(A^{-} \Lambda^{+}-2 \Lambda\right)}{3\left(1+A^{-}\right)} .
$$

The stress-continuity condition is described with $v_{i}=v_{B}^{(i)}$. The two schemes $\mathrm{BF}^{ \pm}$are

$$
\begin{gathered}
\mathrm{BF}^{-}: A^{+}=0, \quad \alpha^{+}=1, \quad \alpha^{-}=2, \\
\mathrm{BF}^{+}: A^{-}=0, \quad \alpha^{+}=1+\frac{B(8 \Lambda-3)}{3(4+B)}, \quad \alpha^{-}=\frac{16}{3} \Lambda .
\end{gathered}
$$

The $\mathrm{BF}^{-}$has the same coefficients as the $\mathrm{BF}$ for $\Lambda=\frac{3}{8}$. Requiring $\alpha^{+}=1$ in Eq. (B13) we come to solution (B14), which yields $\alpha^{-}=2$; that is, we return to the BF scheme with $\Lambda=\frac{3}{8}$. In turn, requiring $\alpha^{-}=1$ in Eq. (B13), we get
$\alpha^{+}=\frac{8+B}{8+2 B}$; that is, we return to the $\mathrm{BF}^{+}$scheme (B15) with $\Lambda=\frac{3}{16}$. Therefore, there is no solution for the three parameters $\left\{\Lambda, A^{+}, A^{-}\right\}$where the two coefficients $\alpha^{ \pm}$are equal to 1 and $\delta_{\star}\left(A^{ \pm}\right)=0$. 
[1] A. D. Khalili, J.-Y. Arns, F. Hussain, Y. Cinar, W. Pinczewski, C. H. Arns, Permeability upscaling for carbonates from the pore scale by use of multiscale X-Ray-CT images, SPE Reservoir Evaluation \& Engineering 16, 353 (2013).

[2] B. Zinszner, F.-M. Pellerin, and T. Jones, A Geoscientist's Guide to Petrophysics (Technip Editions, Paris, 2007).

[3] J. Gao, H. Xing, Z. Tian, and H. Muhlhaus, Lattice Boltzmann modeling and evaluation of fluid flow in heterogeneous porous media involving multiple matrix constituents, Comput. Geosci 62, 198 (2014).

[4] M. Zalzale, K. L. Scrivener, and P. J. McDonald, A 3D lattice Boltzmann effective media study: understanding the role of C$\mathrm{S}-\mathrm{H}$. and water saturation on the permeability of cement paste, Model. Sumul. Mater. Sci. Eng. 21, 085016 (2013).

[5] R. Li, S. Yang, J. Pan, G. G. Pereira, J. A. Taylor, B. Clennel, and C. Zou, Lattice Boltzmann modeling of permeability in porous materials with partially percolating voxels, Phys. Rev. E 90, 033301 (2014).

[6] A. D. Khalili, S. Yanici, Y. Cinar, and C. H. Arns, Formation factor for heterogeneous carbonate rocks using multi-scale X-ray CT Images, in SPE Kuwait International Petroleum Conference and Exhibition (Society of Petroleum Engineers, Kuwait, 2012).

[7] H. C. Brinkman, A. calculation of the viscous force exerted by a flowing fluid on a dense swarm of particles, Appl. Sci. Res. A 1, 27 (1947).

[8] R. C. Gilver and S. A. Altobelli, A determination of the effective viscosity for the Brinkman-Forchheimer flow model, J. Fluid Mech. 258, 355 (1994).

[9] J. L. Auriault, On the domain of validity of Brinkman equation, Transp. Porous Med. 79, 215 (2010).

[10] J. Koplik, H. Levine, and A. Zee, Viscosity renormalization in the Brinkman equation, Phys. Fluids 26, 2864 (1983).

[11] N. Martys, D. P. Bentz, and E. J. Garboczi, Computer simulation study of the effective viscosity in Brinkman's equation, Phys. Fluids 6, 1434 (1994).

[12] A. Nabovati and C. H. Amon, Hydrodynamic boundary condition at open-porous interface: A pore-level lattice Boltzmann study, Transp. Porous Med. 96, 83 (2012).

[13] G. S. Beavers and D. D. Joseph, Boundary conditions at a naturally permeable wall, J. Fluid Mech. 30, 197 (1967).

[14] W. Jäger, A. Mikelic, and N. Neuss, Asymptotic analysis of the laminar viscous flow over a porous bed, SIAM J. Sci. Comput. 22, 2006 (2001).

[15] J. A. Ochoa-Tapia and S. Whitaker, Momentum transfer at the boundary between a porous medium and a homogeneous fluid. I. Theoretical development, Int. J. Heat Mass Transf. 38, 2635 (1995).

[16] P. G. Saffman, On the boundary condition at the surface of a porous medium, Studies Appl. Math. 50, 93 (1971).

[17] F. J. Valdés-Parada, J. Alvarez-Ramírez, B. Goyeau, and J. A. Ochoa-Tapia, Computation of jump coefficients for momentum transfer between a porous media and a fluid using a closed generalized transfer equation, Transp. Porous Med. 78, 439 (2009).

[18] J. A. Ochoa-Tapia and S. Whitaker, Momentum transfer at the boundary between a porous medium and a homogeneous fluid. II. Comparison with experiment, Int. J. Heat Mass Trans. 38, 2647 (1995).
[19] V. Laptev, Numerical solution of coupled flow in plain and porous media, Ph.D. thesis, TU Kaiserslautern, 2003.

[20] H. Bai, P. Yu, S. H. Winoto, and H. T. Low, Lattice Boltzmann method for flows in porous and homogeneous fluid domains coupled at the interface by stress jump, Int. J. Num. Methods Fluids 60, 691 (2008).

[21] M. Dabrowski, M. Krotkiewski, and D. W. Schmid, MILAMIN: Matlab-based finite element method solver for large problems, Geochem. Geophys. Geosyst. 9, Q04030 (2008).

[22] A. Hannukainen, M. Juntunen, and R. Stenberg, Computations with finite element methods for the Brinkman problem, Comput. Geosci. 15, 155 (2011).

[23] Comsol A. COMSOL multiphysics user's guide. Version July; 2013.

[24] B. P. Lamichhane, A new finite element method for DarcyStokes-Brinkman Equations, ISRN Computational Mathematics 2013, 798059 (2013).

[25] D. M. Freed, Lattice-Boltzmann method for macroscopic porous media modeling, Int. J. Mod. Phys. C 9, 1491 (1998).

[26] M. Spaid and F. Phelan, Lattice Boltzmann method for modeling microscale flow in fibrous porous media, Phys. Fluids 9, 2468 (1997).

[27] Z. Guo and C. Zhao, Lattice Boltzmann model for incompressible flows through porous media, Phys. Rev. E 66, 036304 (2002).

[28] X. Nie and N. S. Martys, Breakdown of Chapman-Enskog expansion and the anisotropic effect for lattice-Boltzmann models of porous media, Phys. Fluids 19, 011702 (2007).

[29] I. Ginzburg, Consistent lattice Boltzmann schemes for the Brinkman model of porous flow and infinite Chapman-Enskog expansion, Phys. Rev. E 77, 066704 (2008).

[30] A. Zarghami, C. Biscarini, S. Succhi, and S. Ubertini, Hydrodynamics in porous media: A finite volume lattice Boltzmann study, J. Sci. Comput. 59, 80 (2014).

[31] Q. Kang, D. Zhang, and S. Chen, Unified lattice Boltzmann method for flow in multiscale porous media, Phys. Rev. E 66, 056307 (2002).

[32] O. Dardis and J. McCloskey, Lattice Boltzmann scheme with real numbered solid density for the simulation of flow in porous media, Phys. Rev. E 57, 4834 (1998).

[33] M. C. Sukop and D. T. Thorne, Lattice Boltzmann Modeling: An Introduction for Geoscientists and Engineers (Springer, Berlin, 2006).

[34] H. Yoshida and H. Hayashi, Transmission-reflection coefficient in the lattice Boltzman method, J. Stat. Phys. 155, 277 (2014).

[35] Y. I. Chen and K. Q. Zhu, A. study of the upper limit of solid scatters density for gray lattice Boltzmann method, Acta Mech. Sin. 24, 515 (2008).

[36] Y. Gao and M. M. Sharma, A LGA model for fluid in heterogeneous porous media, Transp. Porous Med. 17, 1 (1994).

[37] S. D. C. Walsh, H. Burwinkle, and M. O. Saar, A. new partial bounce back lattice Boltzmann method for fluid flow through heterogeneous media, Comput. Geosci. 36, 1186 (2009).

[38] J. Zhu and J. Ma, An improved gray lattice Boltzmann model for simulating fluid flow in multi-scale porous media, Adv. Water Resour. 56, 61 (2013).

[39] F. R. Phelan and G. Weis, Analysis of transverse flow in aligned fibrous porous media, Composites A 27A, 25 (1996). 
[40] L. Talon, J. Martin, N. Rakotomalala, D. Salin, and Y. C. Yortsos, Lattice BGK simulations of macrodispersion in heterogeneous porous media, Water Resour. Res. 39, 1135 (2003).

[41] S. D. C. Walsh and M. O. Saar, Macroscale lattice-Boltzmann methods for low Peclet number solute and transport in heterogeneus porous media, Water Resour. Res. 46, 1 (2010).

[42] Y. Qian, D. d'Humières, and P. Lallemand, Lattice BGK models for Navier-Stokes equation, Europhys. Lett. 17, 479 (1992)

[43] D. d'Humières and I. Ginzburg, Viscosity independent numerical errors for lattice Boltzmann models: From recurrence equations to "magic" collision numbers, Comp. Math. Appl. 58, 823 (2009).

[44] I. Ginzburg and D. d'Humières, Multi-reflection boundary conditions for lattice Boltzmann models, Phys. Rev. E 68 , 066614 (2003)

[45] S. Khirevich, I. Ginzburg, and U. Tallarek, Coarse- and fine-grid numerical behavior of MRT/TRT Lattice-Boltzmann schemes in regular and random sphere packings, J. Comp. Phys. 281, 708 (2015).

[46] L. Talon, D. Bauer, N. Gland, S. Youssef, H. Auradou, and I. Ginzburg, Assessment of the two relaxation time lattice Boltzmann scheme to simulate stokes flow in porous media, Water Resour. Res. 48, W04526-13 (2012).

[47] I. Ginzburg, Lattice Boltzmann modeling with discontinuous collision components: Hydrodynamic and advection-diffusion equations, J. Stat. Phys. 126, 157 (2007).

[48] I. Ginzburg, F. Verhaeghe, and D. d'Humières, Two-relaxationtime lattice Boltzmann scheme: About parametrization, velocity, pressure and mixed boundary conditions, Commun. Comput. Phys. 3, 427 (2008).

[49] I. Ginzburg, Comment on "An improved gray lattice Boltzmann model for simulating fluid flow in multi-scale porous media": Intrinsic links between LBE Brinkman schemes, Adv. Water Resource, doi:10.1016/j.advwatres.2014.05.007.

[50] I. Ginzburg, Truncation errors, exact and heuristic stability analysis of two-relaxation-times lattice Boltzmann schemes for anisotropic advection-diffusion equation, Commun. Comput. Phys. 11, 1439 (2012).

[51] L. Gelhar and C. Axness, Three-dimensional stochastic analysis of macrodispersion in aquifers, Water Resour. Res. 19, 161 (1983).

[52] N. H. Pham, R. S. Voronov, N. R. Tummala, and D. V. Papavassiliuo, Bulk stress distributions in the pore space of sphere-packed beds under Darcy-flow conditions, Phys. Rev. E 89, 033016 (2014).

[53] M. I. Shwidler, Stochastic Hydrodynamics of Porous Medium (in Russian) (Nedra, Moscow, 1985).

[54] A. Vikhansky and I. Ginzburg, Taylor dispersion in heterogeneous porous media: Extended method of moments, theory, and modeling with two-relaxation-times lattice Boltzmann scheme, Phys. Fluids 26, 022104 (2014).

[55] D. d'Humières, I. Ginzburg, M. Krafczyk, P. Lallemand, and L.-S. Luo, Multiple-relaxation-time lattice Boltzmann models in three dimensions, Phil. Trans. R. Soc. Lond. A 360, 437 (2002).

[56] G. Silva and I. Ginzburg, The permeability and quality of velocity field in a square array of solid and permeable cylindrical obstacles with the TRT-LBM and FEM Brinkman-schemes, (unpublished).

[57] U. Scheven, D. Verganelakis, R. Harris, M. Johns, and L. Gladden, Quantitative nuclear magnetic resonance measurements of preasymptotic dispersion in flow through porous media, Phys. Fluids 17, 7107 (2005)

[58] J. Yang, J. Crawshaw, and E. S. Boek, Quantitative determination of molecular propagator distributions for solute transport in homogeneous and heterogeneous porous media using lattice Boltzmann simulations, Water Resour. Res. 49, 8531 (2013).

[59] D. Zhang and Q. Kang, Pore scale simulation of solute transport in fractured porous media, Geophys. Res. Lett. 31, L12504 (2004).

[60] G. Thömmes, J. Becker, M. Junk, A. K. Vaikuntama, D. Kehrwalda, A. Klar, K. Steiner, and A. Wiegmann, Numerical investigation of a combined lattice Boltzmann-level set method for three-dimensional multiphase flow, Int. J. Comput. Fluid Dyn. 23, 687 (2009).

[61] I. Ginzburg and D. d'Humières, Lattice Boltzmann and analytical modeling of flow processes in anisotropic and heterogeneous stratified aquifers, Adv. Water Res. 30, 2202 (2007).

[62] L. Li, R. Mei, and J. F. Klausner, Conjugate heat and mass transfer in the lattice Boltzmann equation method, Phys. Rev. E 89, 043308 (2014).

[63] H. Yoshida, T. Kobayashi, H. Hayashi, T. Kinjo, H. Washizu, and K. Fukuzawa, Boundary condition at a two-phase interface in the lattice Boltzmann method for the convection-diffusion equation, Phys. Rev. E 90, 013303 (2014). 